\title{
Atlas of Atomic Spectral Lines of Neptunium Emitted by an Inductively Coupled Plasma
}

by

E. L. DeKalb and M. C. Edelson

Ames Laboratory

lowa State University

Ames, lowa $50011-3020$

Date transmitted: August 1987 
ATLAS OF ATONIC SPECTRAL LINES OF NEPTUNIUM EMITIED BY AN INDUCTIVELY COUPLED PLASHA

by

E.L. DeKalb and M.C. Edelson

\author{
Ames Laboratory ${ }^{*}$ \\ Iowa state University \\ Ames, IA 50011
}

Date of transmission: August 1987

"Operated by Iowa State University for

the U.S. Department of Energy under Contract

No. W-7405-Eng-82, The work was supported by the office of Safeguards and security of the

U.S. Department of Energy. 


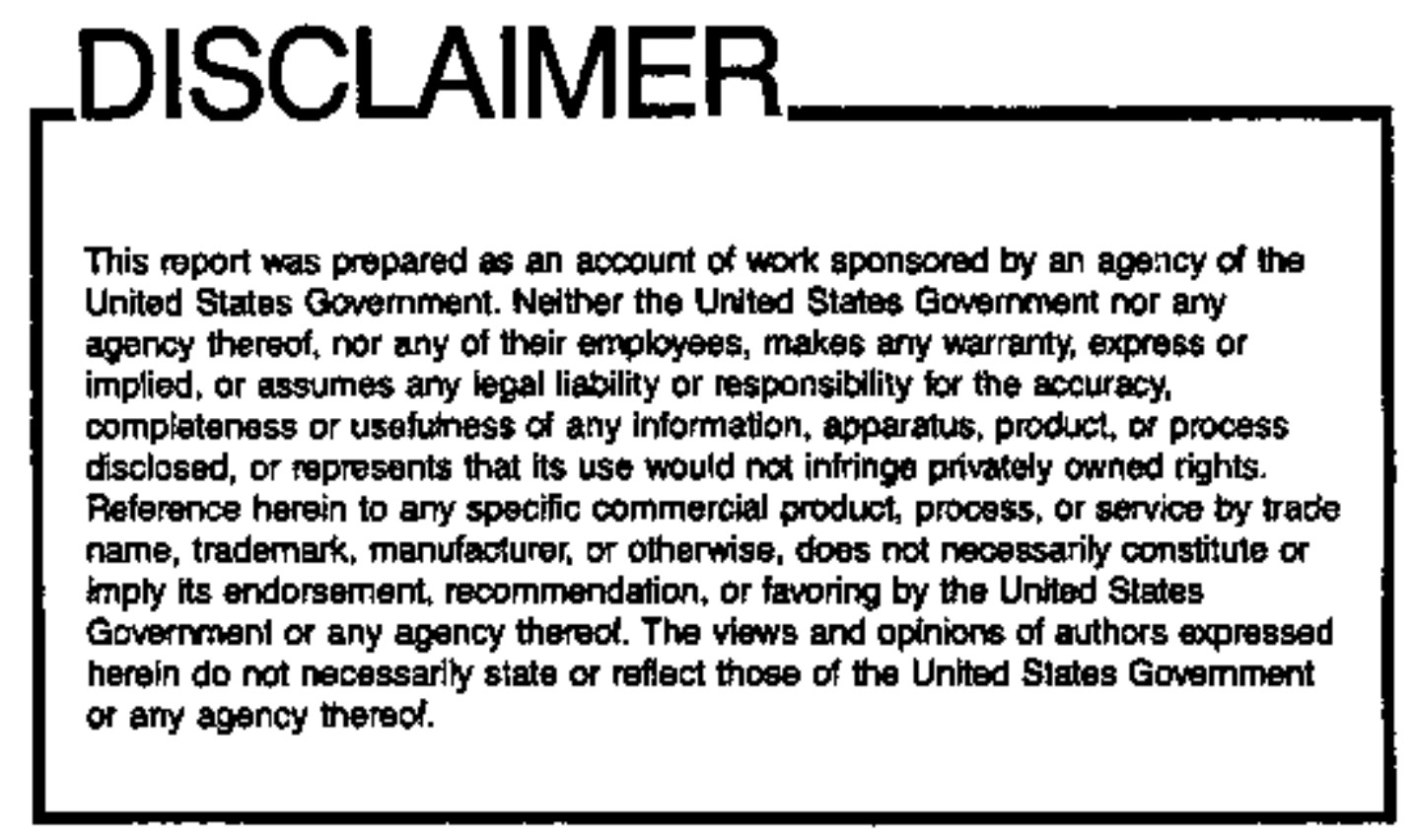

Printed in the United States of America

Available from

National Technical Intormation Service

U.S. Department of Commerce

5265 Port Royal Road

Springfieid, VA 22161 
TABLE OF CONTENTS

TABLE OF CONTENTS

11

ABSTRACT

i11

INTRODUCTION

1

APPARATUS AND PROCEDURES

RESWLTS AND DISCDSSION

10

REFERBNCBS

13

APPENDIX I

15

DISTRIBUTION LIST

132 


\section{ABSTRACT}

Opticel emisgion spectra from high-purity Np-237 were generated with a glovebox-enclosed 1nductively coupled plisma (ICP) source. Spectra covering the $230-700 \mathrm{~nm}$ waveleogth range are presented along whth general cowmentary on the methodology used in collecting the data. 
INIRODUCTION

The Ames Laboratory Nhclear Safeguards and Security Program has been charged with the task of developing optical spectroscopic methods to analyze the composition of spent nuclear fuels. Such materials are highly radioactive even after prolonged "cooling" and are chemically complex. Neptunium (Np) is a highly toxic by-procuct of muclear power generation and is found, in low abundance, in spent nuclear fuels. This atlas of the optical emission spectrum of $\mathrm{NP}$, as produced by an inductively coupled plasma (ICP) spectroscopic source, is part of a general survey of the ICP emission spectra of the actinide elements.

The ICP emission spectrum of the actinides originates almost exclusively from the electronic relaxation of excited, singly ionized species. Spectral data on the Np ion emission spectrum (1.e., the Np II spectrum) have been reported by Tomkins and Fred [1] and Haaland [2]. Tomkins and Fred excited the Np II spectrum with a Cu spark discharge and identified $114 \mathrm{~Np}$ lines in the 265.5 - 436.3 rm spectral range. Hadand, who corrected some spectral line misidentifications in the work of Tonkins and Fred, utilized an enclosed Au spack discharge to excite the Np II spectrum and reported $203 \mathrm{NP}$ Iines within the $265.4-461.0 \mathrm{rm}$ wavelength range.

Frea, Tomins, Blaise, Camus, and Verges [3] published a comprehensive listing of the atomic spectrum of Np (i.e., the Np I spectrum) in the $257.5-2630 \mathrm{~nm}$ wavelength range excited by an electrodeless discharge, and sovey and steers [4], who also used an electrodeless discharge tube to excite the Np I spectrum, recorded the Np spectrum over the $890-2240 \mathrm{~mm}$ range. 
To our knowledge, a comprehensive listing of the Np spectral emission lines protuced by an ICP excitation source has not been reported in the literature. Work has been done at various laboratories to determine the most Bensitive Np emission lines from an ICP source. During an early stage of this work, one of the authors obtained information [5] about the detection limits of several intense Np lines enitted by an ICP source operated at Rockwe11 Hanford Operations, Fichland, WA.

\section{APPARATUS and PROCEDURES}

Axparatus: The data presented herein were collected with an ICP apparatus specially adapted for glovebox operation $[6,7]$. The ICP operating conditions are listed in Table 1.

TABLE 1. ICP OPERATING CONDITIONS

\begin{tabular}{ll}
\hline Eorward R.F. Power & $900 \mathrm{w}$ \\
Reflected Power & $<5 \mathrm{w}$ \\
Argon Flow Rates & $17 \mathrm{1}$ /min \\
$\quad$ Outer Gas & For igmition only \\
$\quad$ Nebulizer Gas & $0.95 \mathrm{i} / \mathrm{min}$ \\
Sample Uptake Rate & $0.25 \mathrm{ml} /$ min \\
Observation Height & $15 \mathrm{~mm}$ above load coil \\
Integration Time & see text below \\
\hline
\end{tabular}

Some of the operating conditions were dictated by the limitations of glovebox operation rather than by spectroscopic considerations. For example, the power applied at the ICP torch was restricted to $900 \mathrm{~W}$ forward power to minimize the heat input to a nearby air filtration device, and the torch-toslit distance was set at $\sim 1.7 \mathrm{~m}$ to permit the further isolation of the 
enclosed ICP in a separately ventilated room. A concentric nebulizer with a nominal solution uptake rate of $\sim 0.3$ to $0.5 \mathrm{ml}$ /minute was chosen for this work. A peristaltic pump was used to further limit the solution uptake rate to $0.25 \mathrm{ml} / \mathrm{min}$ and thereby to reduce the amount of liquid waste generated during ICP operation.

The instrumentation used is described in Table 2, A schenatic diagram of the experimental lay-out is included here as Fig. 1. The imaging optics resulted in an image demagnification at the entrance slit of $\sim_{3 x}$.

TARLE 2. INSTMUNBNTAMION

A. PONER and NEBULIZER SYSTEN Generator Nebulizer

Torch Spray Chamber Peristeltic Pump Nass Flow Controllers

Type HFS 3000D (Plasma-Therm)

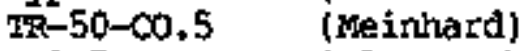

T 1.5 (Plasma-Therm) - (Plasma-Therm)

Minipuls 2 (Gilson Med, Elec) (3-channel) (NKS)

B. SPECTROMETER Grating

Entrance slit Filter

$1.50 \mathrm{~m}, \mathrm{f} / 14 \mathrm{THR} 1500$ (Jobin-Yvon)

2400 groove/mm (Jobin-Yvon)

(Waster holographic grating)

$0.015 \mathrm{~mm}$

Schott \#GG 400 for $500-700$ in

C. SIGNAL DETECTION ELECTRONICS

OMA.

Nodel 1450 (BGGG/FAR)

PDA Detector

MOdel 1453 (EGGG/PAR)

D. COMPUTER SYSTEA

CFU

$11 / 73$ (Digital Equipuent)

Graphics Terainal

Operzting System

Hodel 550

(Visual Technology)

ET-11 V.5.2 (Digital Equipment)

Software

plotter

Ames Laboratory

Model 9872s (Hewlett Packard) 


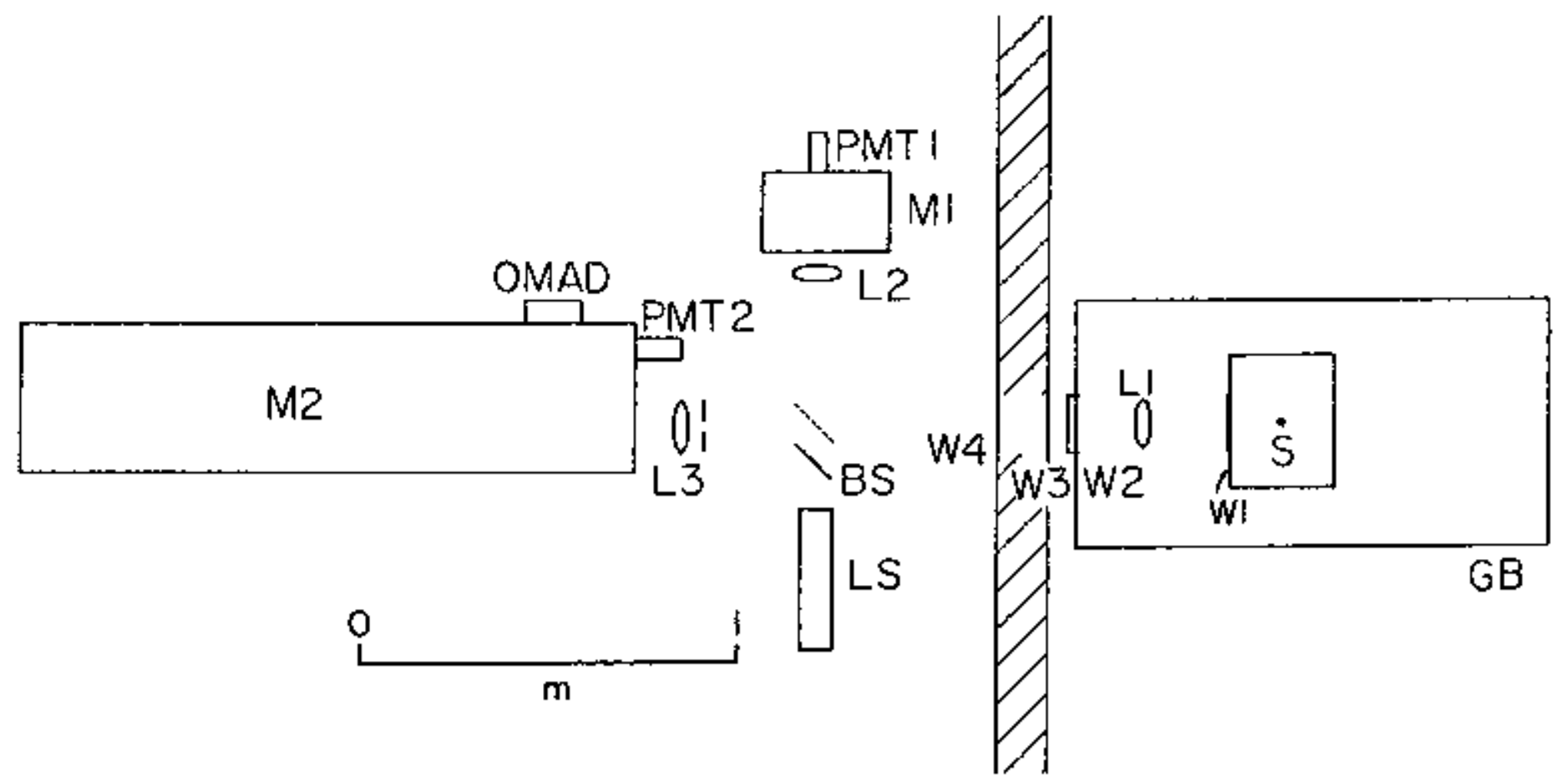

F1g. 1. Experimental lay-out. GB = statnless steel glovebox, $\mathrm{S}=$ ICP source, W1-W4 = fused silica windows, L1-L3 = $25 \mathrm{~mm}$ diameter fused silica lenses, LS = He-Ne al1gnment laser,

Ml-M2 = monochromatoxs, BS = fused słlica beamsplicter (R - -8\%), FWT 1-2 = photomultipliers, OHAD = optlcal multichannel analyzer detector. A more complete equipment specification is provided in Table 2 . 
Reagents: High purity $237 \mathrm{NpO}_{2}$, obtained from oak Ridge National Laboratory, was dissolved in $\mathrm{HNO}_{3}$ to prepare a $0.4 \mathrm{mg} / \mathrm{ml}{ }^{237} \mathrm{~Np}$ solution. This solution was introduced into the ICP torch to generate the emission spectra contained in this report.

Analysis of the starting material by spark somrce mass spectroscopy at Oak Ridge National Laboratory indicated that the Np sample contained less than 65 ppm total impurities (see Table 3).

TABLE 3. Np-237 IMPURITY ANALYSIS

\begin{tabular}{|c|c|}
\hline $\begin{array}{l}\text { Boron } \\
\text { Calcium } \\
\text { Chlorine } \\
\text { Chromium } \\
\text { Iron } \\
\text { Potassium } \\
\text { Sodium } \\
\text { Phosphorus } \\
\text { Plutonium } \\
\text { Sulfur } \\
\text { Silicon } \\
\text { Thorimm } \\
\text { Uranium } \\
\text { Zinc } \\
\text { Zirconium }\end{array}$ & $\begin{array}{l}0.1 \\
2 \\
10 \\
0.3 \\
3 \\
0.3 \\
2 \\
0.4 \\
<3 \\
3 \\
3 \\
<3 \\
5 \\
0.3 \\
0.2\end{array}$ \\
\hline
\end{tabular}

Even though the radloactive decay of the Np-237 atom is primarily through alpha-particle emission and $\mathrm{Np}-237$ has a relatively long half life of $2 \times 10^{6}$ years and hence has a low speciflc activity, it was found that our sample emitted gamma radiation. This was from Pa-233, a short-lived daughter product 
of $\mathrm{Np}-237$, that was in secular equilibrium with the parent nucleus. To reduce operator exposure to the penetrating gama-radiation, the bottle containing the Np stock solution was placed into a Pb covering. Laboratory personnel were also issued dosimeter badges to monitor exposure to gamma radiation. The garmin radiation dose rate in the Imodiate vicinity of the glovebox was measured by Health Physics personnel and was not deemed to be a health hazard (see Table 4).

TABLE 4. ALPHA FACILITY RADIATION SURVEY

ISOTOPE; Np-237/ $\mathrm{Aa}-233$

INSTRUMDAT; FUG

ROOM BACKGROHND;

BOX WINDOW- CENTER AT 4" FROM BOT;

LEFT GLOVE FORT AT SUREACE;

BOX BET L AND Y PORT AT SURFACE;

CENIER GLONE PORT AT SUJRFACE

CENTER GLONE PORT AT $3^{n}$ IN;

BOX BET M AND R RORT AT SURFACE;

RIGET GLOVE PORT AT SURFACE;

BOX TOP AT SURFACE;

EXHAUST FILTER AT SURFACE;

2ND EXTAUST RILTER AT SURFACE;

LOWER REAR OF BOX AT SUFFACE;
$.02 \mathrm{MR} / \mathrm{H}$

$.30 \mathrm{NR} / \mathrm{A}$

$.20 \mathrm{MR} / \mathrm{H}$

$.18 \mathrm{MR} / \mathrm{H}$

$.43 \mathrm{KR} / \mathrm{H}$

$.80 \mathrm{MR} / \mathrm{H}$

$.30 \mathrm{MR} / \mathrm{H}$

$.27 \mathrm{MP} / \mathrm{H}$

$.04 \mathrm{MP} / \mathrm{H}$

.14 WAR

.02 吥/

.15 䜣/ $/ \mathrm{H}$

Procedures: A photodiode artay, optical multichannel analyzer (FDA-OMA) was used to record the optical emission of Np from 230 to $700 \mathrm{fm}$. This device was mounted on the photographic exit port of the THR1500 monochromator and, because this port was located perpendicular to the usual optical path, a 
mirror was used to divert the dispersed radiation to the PDA. The monochromator was used in the "single-pass" mode. In this mode, with a 2400 grooves/mm grating, the resolution of the systern was $\sim 6$ pm (at $300 \mathrm{~nm}$ ) and the spectral coverage was ${ }^{-} 6 \mathrm{~nm}$. The maximan useful integration time per measurement was detecmined to be 1 second; the photodiode detector saturated for integration times greater than 15 . The find oma spectra were prepared by sutaring a number of individual 1 second PDA exposures for each wavelength range recorded. The exposure conditions used for the various wavelength ranges are collected in Table 5. Two lens positions were used to focus the inage of the plasma source onto the spectrometer entrance slit, one for the visible spectrum and the other for the ultraviolet spectrum. Spectral line intensities were relatively insensitive to changes in the lens position.

TABLE 5. OHA EXPOSURE CONBITIONS

$\begin{array}{lclll}\begin{array}{l}\text { Wavelength } \\ \text { range (nm) }\end{array} & \begin{array}{l}\text { Number of one- } \\ \text { Second Integrations }\end{array} & \begin{array}{l}\text { slit } \\ \text { Widuth }(\mu)\end{array} & \begin{array}{l}\text { Lens } \\ \text { Focus }\end{array} & \begin{array}{l}\text { Optical } \\ \text { Filter }\end{array} \\ 230-300 & 320 & 50 & \text { UV } & \text { none } \\ 300-400 & 160 & 25 & \text { UV } & \text { none } \\ 400-500 & 160 & 25 & \text { visible } & \text { none } \\ 500-700 & 160 & 25 & \text { visible } & \text { GG400 }\end{array}$

To remove second orơer spectral lines, sharp cut optical filter was used for the higher wavelength exposures. The filter had a transmission of less than 18 for wavelengths below $370 \mathrm{~nm}$ and more than 958 for wavelength greater than $500 \mathrm{~nm}$. 
Software was written to link the OMA to a laboratory computer and the ONA spectra were transferred to comguter memory after accuistion. The OMA output to the computer was in tabular form listing pixel number (from 0 to 1023) versus intensity as 'counts'. The counts were linearly related to the diode photocurrent. These spectra were then permanently stored on flexible 51/4" diskettes.

Three aspects of the ond spectral data presented here should be noted. First, the FDA sensitivity varied with wavelength and was at a maximum near $600 \mathrm{~mm}[8]$. No attempt was made to compensate for this sensitivity varlation. Second, the 1024 channel photodiode array consists of alternating p- and $n-s i l i c o n$ regions, both of which are light sensitive. A signal resulting from light falling on an n-region is divided betwen the adjacent pregions. Therefore, even for situations where the spectral bandpass approximated the diode spacing of the detector, as was the case for this work, the Gaussian-like spectral line profile covered portions of several diodes. Thus the measured intensity of a line depended on the position of the line profile relative the $\mathrm{p}$ - and n-regions. No attempt was made to compensate for this spectral line-to-diode registry effect, which has been shown to result in intensity ecrors of as much as 30 s [9]. Finally, the first ${ }^{\prime} 175$ photodiodes (on the low wavelength stde of the PDA) were noted to be less light sensitive than were the remainder of the diodes. This loss of sensitivity was due to a small degree of vignetting in the spectrometer. Usually there was sufficient spectral overlap between successive ip spectral segments so that intensity information could be collected from lines isolated on the unvignetted portion of the diode array. 
Spectral calibration: Haaland's Np line list $[2]$, which includes all of the verified lines from the list of Tonkins and Fred, proved to be useful for calibrating the NP ICP spectrum. For spectral ranges that included lines reported in Haaland's study, those lines were taken as wavelength standards. If available, three lines were used to calibrate each diode array spectral segment. A quadratic fitting function was used in the calibration calculation. When three identified Np lines were not available, a single line was used in conjunction with spectrometer dispersion values obtained auring the preparation of $\mathrm{U}$ and Pu atlases $[10,11]$. For spectral segments that did not include any of Haaland's identified lines, the spectral overlap of adjacent segments was used to provide wavelength markers that could be used for the calibration. Inaccuracies in wavelength assignments were thus passed on to the adjacent segment to compoind the margin of frror for that segment. Wany of the ${ }^{237}$ Np lines show a substantial amount of hyperfine splitting, in some cases more than $0.05 \mathrm{~nm}$ (see Fig. 2). It is not clear whether the wavelengths of such lines listed by Haaland cepresent the strongest line component, or the centroid of the plate blackening. For this atlas, we have assigned hadand's wavelength to the strongest component, or to the ort pixel with the highest intensity when hyperfine splitting was not resolved. This uncertainty could introduce a bias in our wavelength assigmments of up to $0.05 \mathrm{rm}$.

Calibration of $\mathrm{Np}$ wavelengths greater than $440 \mathrm{~nm}$, beyond the range of the published reference spectra, was accomplished by measuring the spectrum of a mixture of $300 \mu \mathrm{g} 237 \mathrm{~Np} / \mathrm{ml}$ and $160 \mu \mathrm{g}{ }^{242} \mathrm{Pu} / \mathrm{ml}$. These spectra were calibrated using the previousiy identified Fu lines and lines of $\mathrm{H}, \mathrm{O}$, and Ar [11]. The stronger Np lines were thus assigned wavelength values, which were subsequently used to calibrate the Np spectral segments. Again, any 


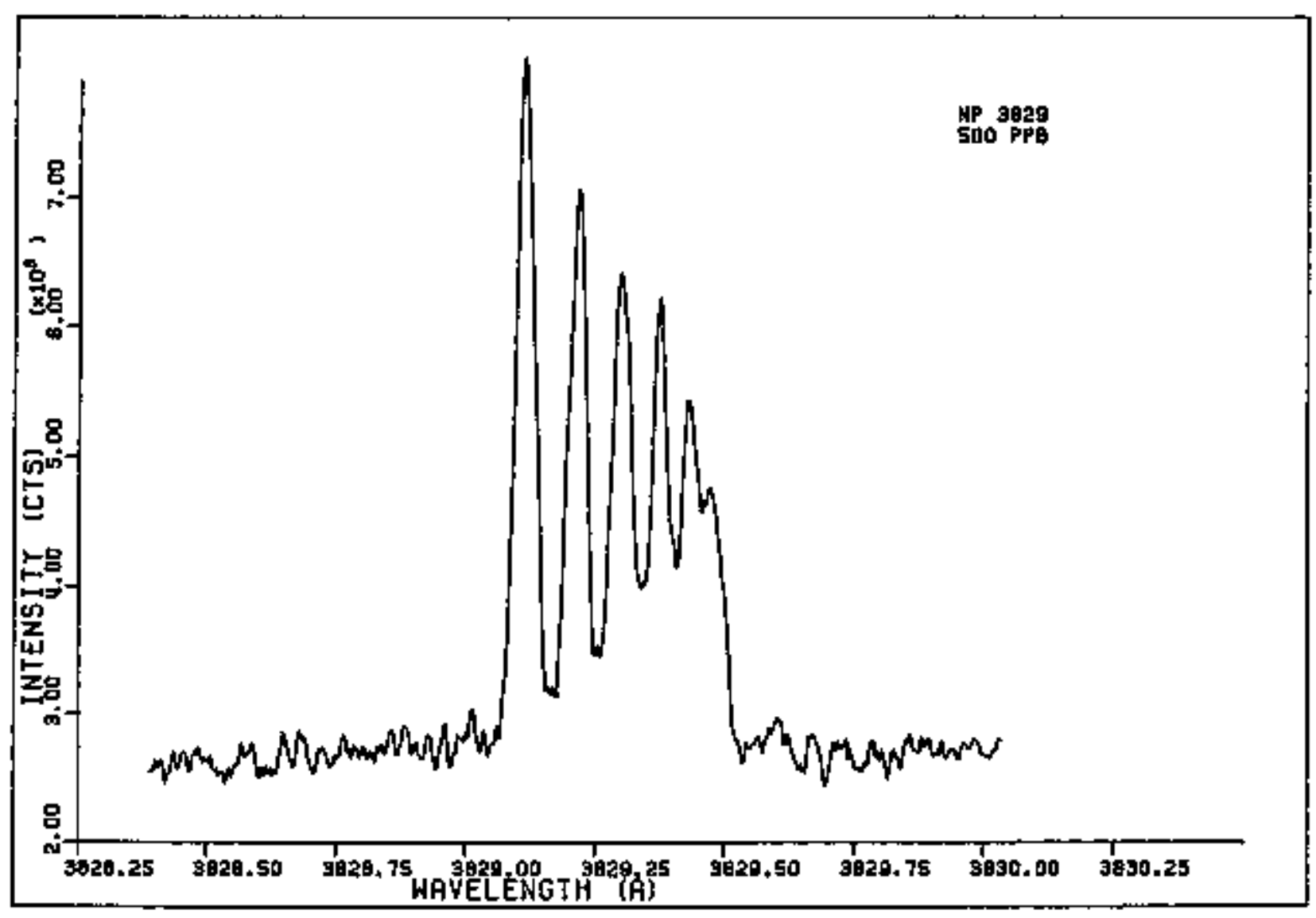

Fig. 2. Np-237 1ine at approwimately 3829.1A showing kyperfine sp1itting of nearly 0.5A. Thig figure was obtained by scanning the spectrometer, in double pass toode, whlle nonftoting the exit slit with a photomultiplier. 
inaccuracies in the Pu wavelengths would be transferred to the $\mathrm{Np}$ Iines. However, the Pu whelengths are belleved to be accurate to within $5 \mathrm{pm}$, although instances of greater inaccuracy are possible.

Np wavelengths below $265 \mathrm{~nm}$ were not previously reported, so calibration of the regions from 230 to $265 \mathrm{~nm}$ was carried out by using the monochromator dispersion (known from previous atlas work [11]), and the central wavelength as indicated by the wavelength counter. For this $35 \mathrm{~nm}$ region, the wavelength calibrations are probably accurate to within $0.2 \mathrm{~nm}$.

\section{RESULTS AND DISCUSSION}

Appendix I consists of 115 wavelength-vs-intensity plots of the $237 \mathrm{~Np}$ OMA data, corrected for detector dark current. These plots cover the spectral region extending from 228 to $700 \mathrm{rm}$.

Tabular data on the pages facing the wovelength-vs-intensity plots list the stronger lines, which are narked on the plots with either a dot of an element symbol (for lines not due to Np enission). The first colum of the tables gives the line wavelength in Angstrom units (1.e., 1 Angstrom unit $0.1 \mathrm{~nm})$. The line positions are generally accurate to within $0.05 \mathrm{~nm}$ for the Np lines. The second column lists the spectral line intensities, in counts. These values have been corrected for detector dark current, but have not been corrected to account for the variation of instrument sensitivity to spectral wavelength. An attermt was made to estimate this variation by recording the spectrum of a guartz-halogen lamp over the 250-700 rim range and dividing the resulting spectrum by the expected "black-boxty" enission intensity from this 
source. The results are shown in Fig. 3, which taust be understood to represent only an approximation of the actual instcument response function.

Few Np lines were found in the spectral region from 198 to $248 \mathrm{~nm}$, possibly because of the poor response of the spectroscopic apparatus at those wavelengths. To compensate for the poor sensitivity, the entrance slit of the monochromator was opened to $0.050 \mathrm{sun}$, and the number of one-second integrations was doubled to 320 .

The spectral regions above $637 \mathrm{~mm}$ were smoothed with running three point averaging procedure to increase their signal-to-noise ratio. This also reduced the apparent maximum intensity of the spectral lines, especially for the sharp lines. The intensity values in the accompanying tables were measured prior to the smoothing procedure, and consequently, the tabular intensities for the regions from 637 to $700 \mathrm{~mm}$ may not agree with the spectral intensities shown in the smoothed plots.

The detection limts of the most prominent $\mathrm{Np}$ lines found in this atlas have been measured with a photomiliplier detector and with the spectrometer in the double gass rode. This was done previously to determine the detection limits of several prominent Pu lines [12]. Table 6 lists the 30 detection limits for the strongest ICP emission lines of Np.

Table 6. Np-237 ICF DETECTION LIKITS

Favelength (nm)

Detection Limit $(\mu \mathrm{g} / \mathrm{I})$

382.91

430.77

25

429.08

37

456.04

416.44

48

386.52

52

60

410.84

75

302.66

405.10 
THR15OQ SENGITIVITY

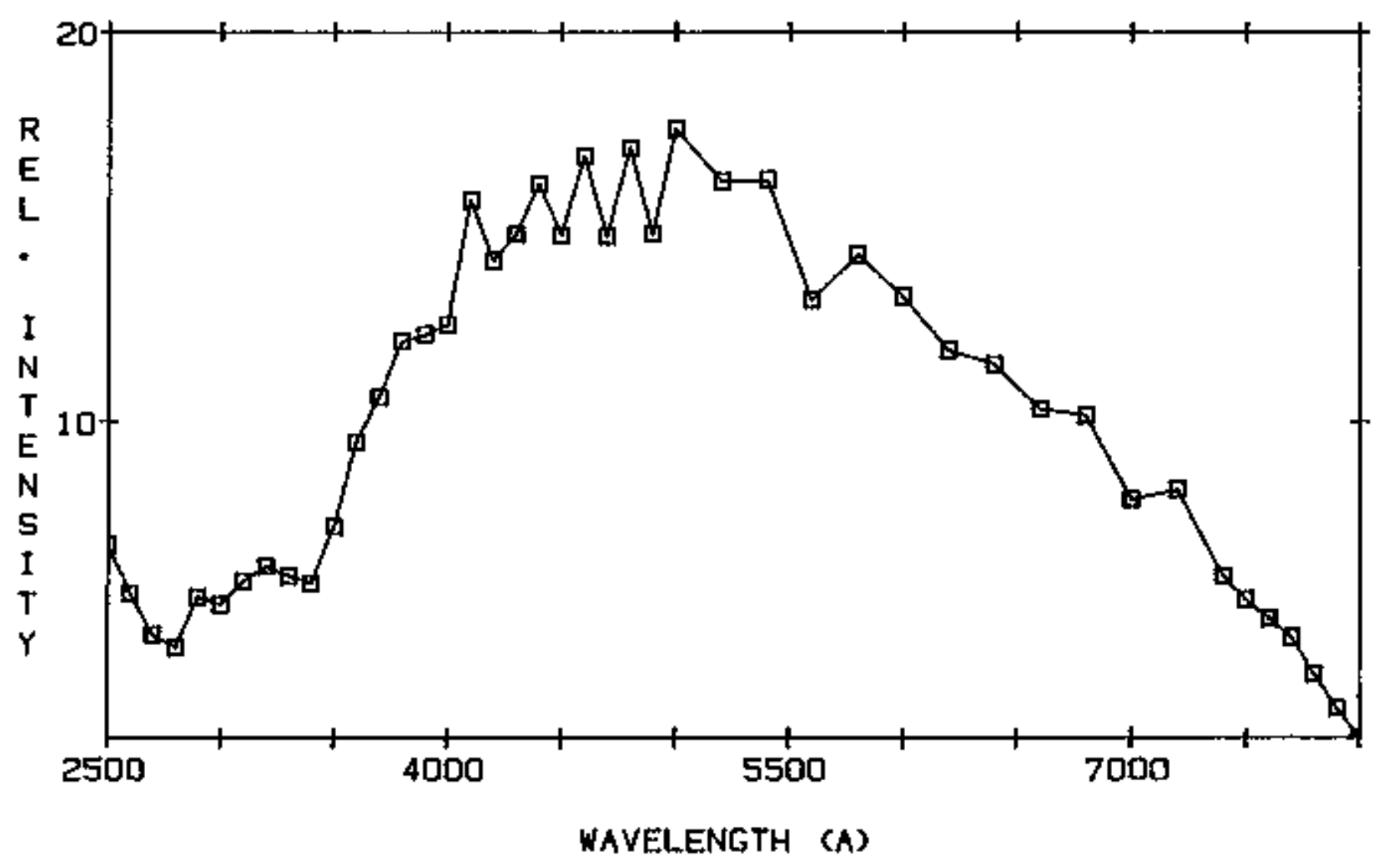

- D- AVE RESPONSE

Fig. 3. Approximate variation of monochromator-ONA Eensitivity with wavelength. Measured using a 1000W quartz-halogen source (Kodel PEL, [01000/4CL], General Electric) operated at $8 A$, with a specified color temperature of $3200 \mathrm{~K}$. Curve shown is the average of measurements with two different lamps. 
FEE'BRENACES

1. P.S. Tomkins and $M$. Fred, "The Spectra of the Heavy kilements,"

J. Opt. Soc. Amer. 39 (1949) 357-362.

2. J. Haaland, "wavelength and Intensity Heasurements in the Spark Excited Spectrum of Neptuniun, "Report KR-1, Rjeller Research Establishment, Norway, 1959 .

3. M. Fred, F.S. Tomkins, J.E. Elaise, F, Camss, and J. Verges, "The Atomic Spectrum of Neptunium," Report ANT -76-68, Argonne National Laboratory, Argonne, IL, May 1976.

4. L. Bovey and E.B.M. Steers, "The Optical Spectra of Some Rare Earth and Transuranic Elements in the 1-3 Micron Region, Part IV: The Spectrum of Neptunium," Report AERE-R3118, United Kingdom Atomic Energy Research Establishment, Harwell, 1959.

5. D. Lowry, Rockwell Hanford Operations, Richland, WA., Private commication.

6. M.C. Edelson and V.A. Fassel, "A New Facility for High Resolution Inductively Coupled Plasma-Atonic Multielement Bmission Spectroscopy of Radloactive Materials," in Analytical Spectroscopy, W.S. Lyon, ed., Elsevier, Amsterdan 1984, pp. $227-233$.

7. M.C. Edelson and E.L. DeKalb, "The Ames Laboratory Facility for the Emission Spectroscopic Study of Alpha-Enitting Radionucleii: The Design and Operating History, " in Enclosed Plasja Spectroscopic Sources for the Anglysis of Toxic and Hazardous Materials (ASTK STP 951), M.C. Edelson and J. Leland Daniel editors, AsTh, Fhiladelphia, to be published in 1987.

8. Model 1450 Optical Multichannel Analyzer Operating and Service Manual, Pig. I-4. DK\&G Princeton Applied Research Corp., Frinceton, N.J. (1982).

9. R.K. Winge, V.A. Fassel, and D.E. Eckels, "Spectral Line-Diode Registry Effects with Photodiode Array Detectors," Appl. Spectrosc. 40 (1986) 461-464.

10. M.C. Edelson, E.L. Dekalb, J. Dougias, and V.A. Fassel, "At.las of Atomic Spectral Lines of Uranium Emitted by an Inductively Coupled Plasma, "Ames Laboratory Report, in preparation.

11. M.C. Edelson, E.L. DeKalb, R.K. Winge, and V.A. Fassel, "Atlas of Atomic Spectral tines of Plutonium Emitted by an Inductively Coupled Plasua," Report IS-4883, Ames Laboratory, Ames, IA, 1986.

12. M.C. Edelson, E.t. Dekalb, R.K. Winge, and V.A. Fassel, "Analytical Atomic Spectroscopy of Plutonium-1. High Resolution Spectra of Plutonium Emitted in an Inductively Coupled Plasma," Spectrochimica Acta 41B (1986) 475-486. 


\title{
APPENDIX I
}

\section{FIGJRES 1-115}

\begin{abstract}
ICP-OWA spectra of a $\stackrel{25}{\approx} \mathrm{mg} / \mathrm{ml} \mathrm{Np}-237$ solution. The spectra are corrected for GA detector dark current contributions, are unmoothed, and represent the summation of 160 one-second integrations (see text for special recording conditions for spectra in the $230-300$ and the $637-700 \mathrm{nrm}$ regions). Please note that the wavelength axis of these figures is labelled in Angstrom units, and that $1 \mathrm{~A}=0.1 \mathrm{~nm}$.
\end{abstract}




$\begin{array}{cc}\lambda(A) & I \\ 2292.7612 & 1969 . \\ 2300.3940 & 2400 . \\ 2325+6621 & 3370 . \\ 2340.6211 & 1668 . \\ 2362.1445 & 2596 . \\ 2366.2129 & 2540 . \\ 2367.5254 & 3023 . \\ 2369.4939 & 2484 . \\ 2371.5938 & 3059 . \\ 2372.2500 & 5043 . \\ 2375.2686 & 2490 . \\ 2378.4839 & 2972 .\end{array}$



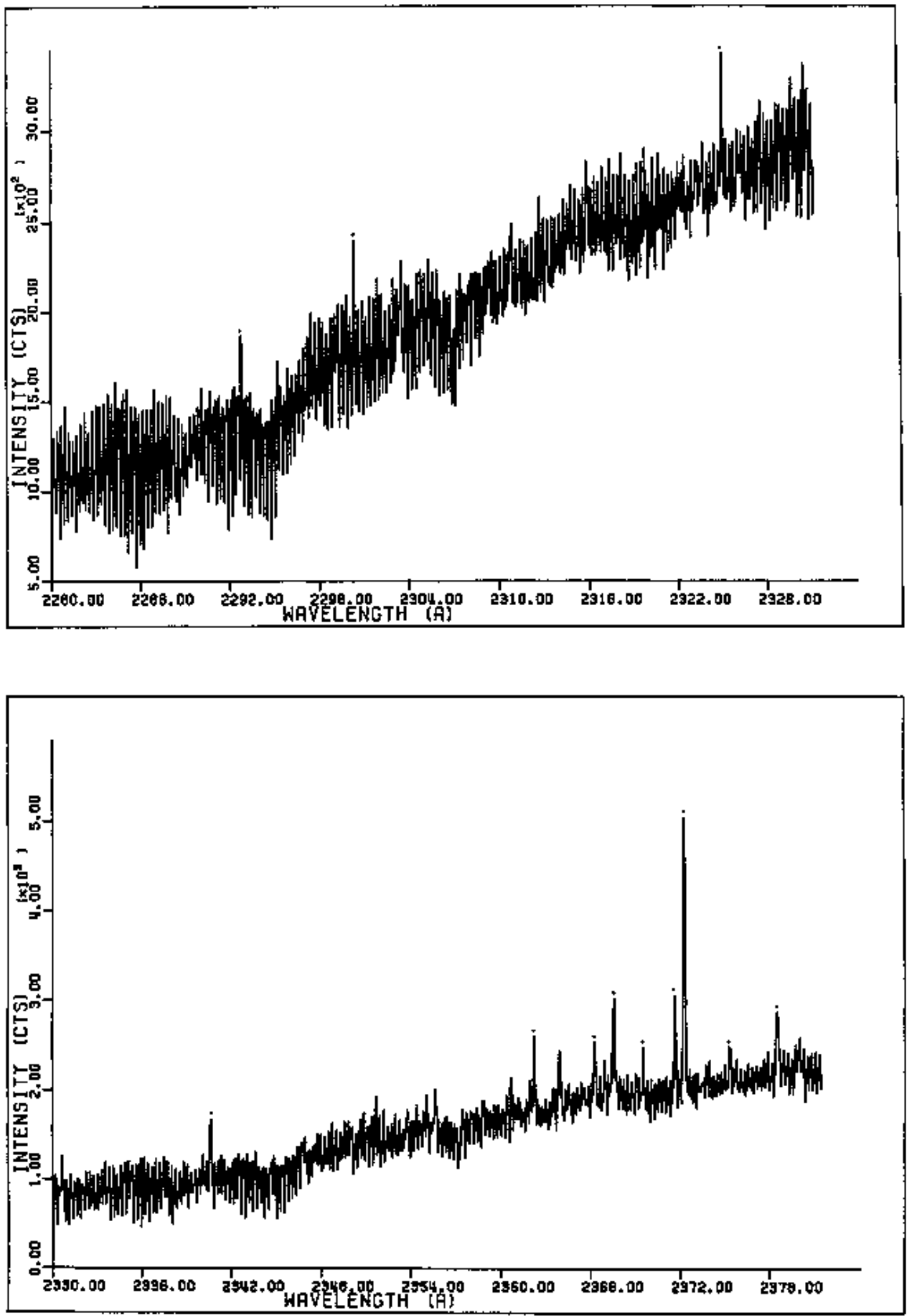


\begin{tabular}{|c|c|}
\hline$\lambda(\mathrm{A})$ & $I$ \\
\hline $2387+8926$ & $\overline{2} 233$. \\
\hline $\begin{array}{l}2402.4224 \\
2405.5640 \\
2413.7451\end{array}$ & $\begin{array}{l}2510 . \\
2524 . \\
2383 .\end{array}$ \\
\hline $\begin{array}{l}2416,8213 \\
2417,2141 \\
2419,7012\end{array}$ & $\begin{array}{l}2467 . \\
3652 . \\
2192 .\end{array}$ \\
\hline $\begin{array}{l}2420.6174 \\
2422.1882 \\
2422.7773\end{array}$ & $\begin{array}{l}2453 . \\
2762 . \\
2387 .\end{array}$ \\
\hline $\begin{array}{l}2424+0964 \\
2427+5552 \\
2429.1260\end{array}$ & $\begin{array}{l}2263 . \\
2336, \\
2441 .\end{array}$ \\
\hline $\begin{array}{l}242 \%+9114 \\
2431.1550 \\
2437.4570\end{array}$ & $\begin{array}{l}2157 . \\
2401 . \\
4049 .\end{array}$ \\
\hline 2442.2239 & 2149 \\
\hline $\begin{array}{l}2442,8770 \\
2446.7949\end{array}$ & $\begin{array}{l}3811 . \\
2264 \text {. }\end{array}$ \\
\hline 2447.0562 & 2825 . \\
\hline $\begin{array}{l}2447.5784 \\
2451.9536\end{array}$ & $\begin{array}{l}2209 . \\
2704 .\end{array}$ \\
\hline 2453.3901 & 3529 \\
\hline $\begin{array}{l}2460+0508 \\
2462+3362\end{array}$ & $\begin{array}{l}2414+ \\
2493 .\end{array}$ \\
\hline 2462.7893 & 2744. \\
\hline $\begin{array}{l}2464,4912 \\
2464,8176\end{array}$ & $\begin{array}{l}3569 . \\
2076 .\end{array}$ \\
\hline 2468.2786 & 2049. \\
\hline 2469.3233 & 3647 \\
\hline 2475.0044 & 2111 \\
\hline $2475+3311$ & 2597 \\
\hline $\begin{array}{l}2476.0493 \\
2476.7676\end{array}$ & 2291 \\
\hline 2478.2695 & $\begin{array}{l}2000 \\
2885\end{array}$ \\
\hline $2479+1836$ & 2901 , \\
\hline
\end{tabular}



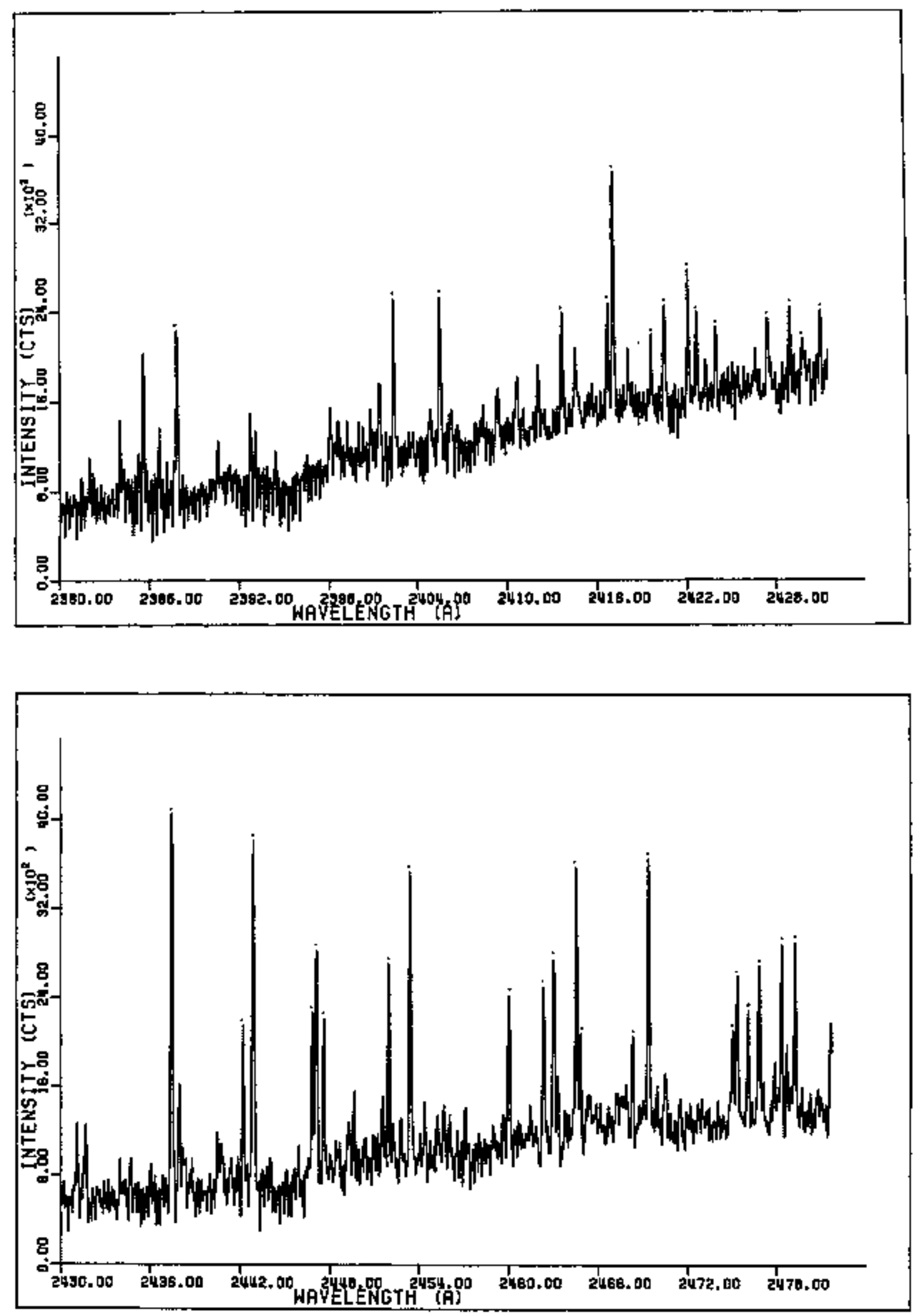


$\begin{array}{cc}\lambda(A) & \text { I } \\ 2484.0356 . & 294 \mathrm{~B} . \\ 2484.9821 & 4781, \\ 2490+4807 & 2973 . \\ 2492.7590 & 8510 . \\ 2498.6831 & 2846 . \\ 2500.4409 & 3766 . \\ 2500+7664 & 7465 . \\ 2504.5422 & 3411 . \\ 2506.1047 & 13680 . \\ 2507.2114 & 2939 . \\ 2507.9974 & 2868 . \\ 2508.6436 & 5066 . \\ 2515.1536 & 9431 . \\ 2515.6091 & 3363 . \\ 2520.2964 & 6045 . \\ 2521.9599 & 2738, \\ 2523.8770 & 2444 . \\ 2526.0903 & 2468 . \\ 2526.3509 & 3596 . \\ 2529.2151 & 5532 . \\ 2529.6057 & 3026 .\end{array}$



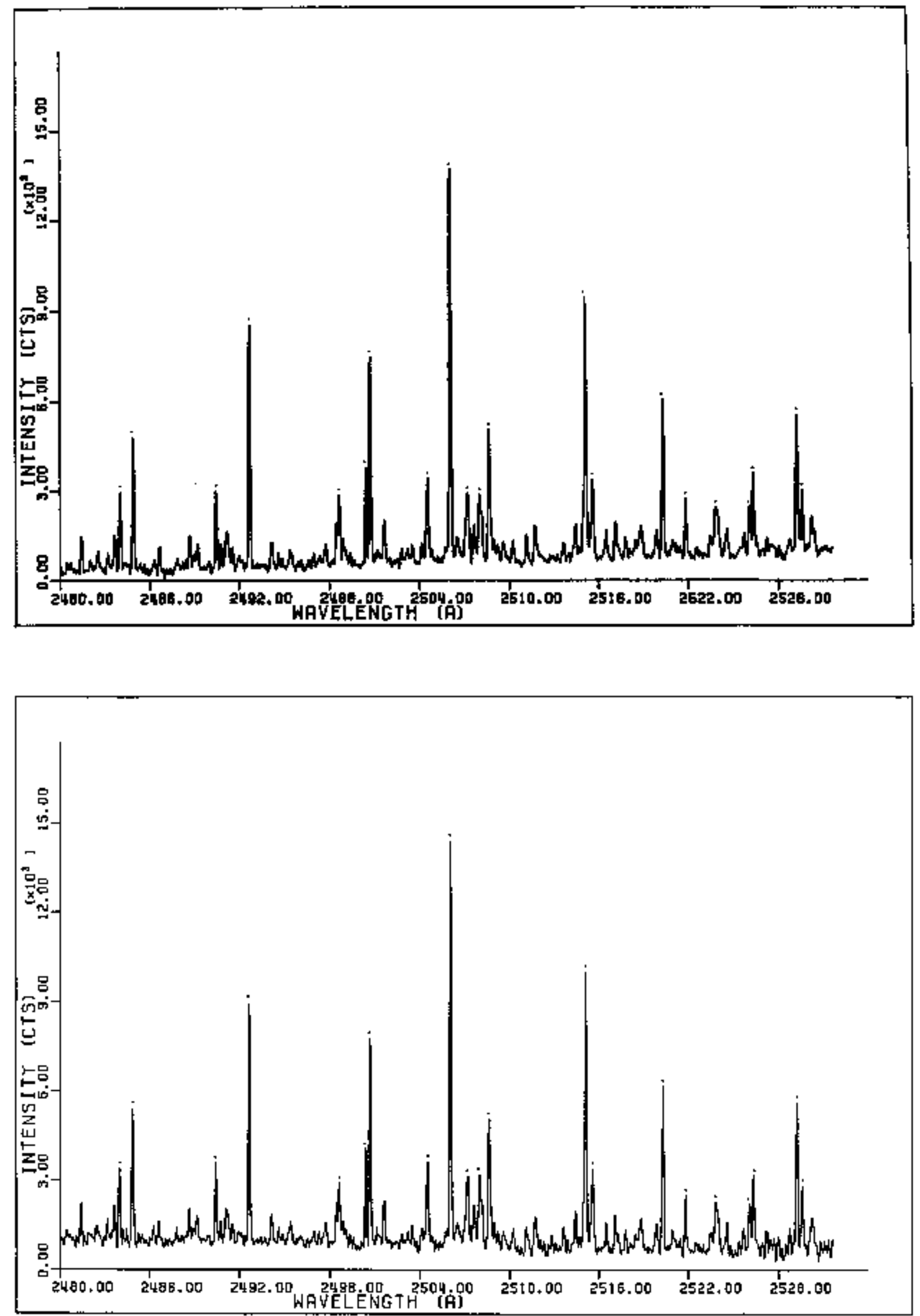


\begin{tabular}{|c|c|}
\hline \multirow[b]{2}{*}{7537801} & $\mathbf{I}$ \\
\hline & 614 \\
\hline 2535.1301 & \\
\hline 2336.0201 & \\
\hline 2537.1450 & 363 \\
\hline 2338.9651 & 13005 \\
\hline $25 \times 2.0652$ & 6183 \\
\hline 346.4402 & 50 \\
\hline 247,4150 & 195 \\
\hline 2051.510 & 3502 \\
\hline 2534,0100 & 4769 \\
\hline 2508.9202 & 4527 \\
\hline 2560.8701 & 82 \\
\hline $\begin{array}{l}2565.6802 \\
256.9802\end{array}$ & 5685 \\
\hline $2573+0251$ & 357 \\
\hline 2575.6902 & 4203. \\
\hline 277.2500 & 4521 . \\
\hline 2591.1326 & s3!, \\
\hline 2583.1416 & 6289. \\
\hline 2585.6699 & 431, \\
\hline 2589.2329 & 5177. \\
\hline $\begin{array}{l}2590.3801 \\
2575.3889\end{array}$ & $\begin{array}{l}5862+ \\
647 \%\end{array}$ \\
\hline 2590.3046 & 9137. \\
\hline 2596,9524 & 499. \\
\hline $\begin{array}{l}2603.9426 \\
2695.3682\end{array}$ & 5446, \\
\hline 2605,6272 & 1974 . \\
\hline 2606.9233 & $245+4$. \\
\hline 2607,3770 & 5359 \\
\hline 2609,0618 & $48 \%$ \\
\hline $2 d t^{3} .92 t d$ & 15927 \\
\hline 2615.6064 & 967 \\
\hline 2620.9202 & 5110 \\
\hline 2622.1514 & 6327 \\
\hline 2623.1062 & 5073. \\
\hline 2623,0362 & 5912 \\
\hline $2624.26 \%$ & 14020 \\
\hline 2628.566 & 4690. \\
\hline 2628.76 & $\begin{array}{l}5064, \\
19610 .\end{array}$ \\
\hline 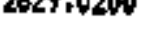 & 19610 \\
\hline
\end{tabular}



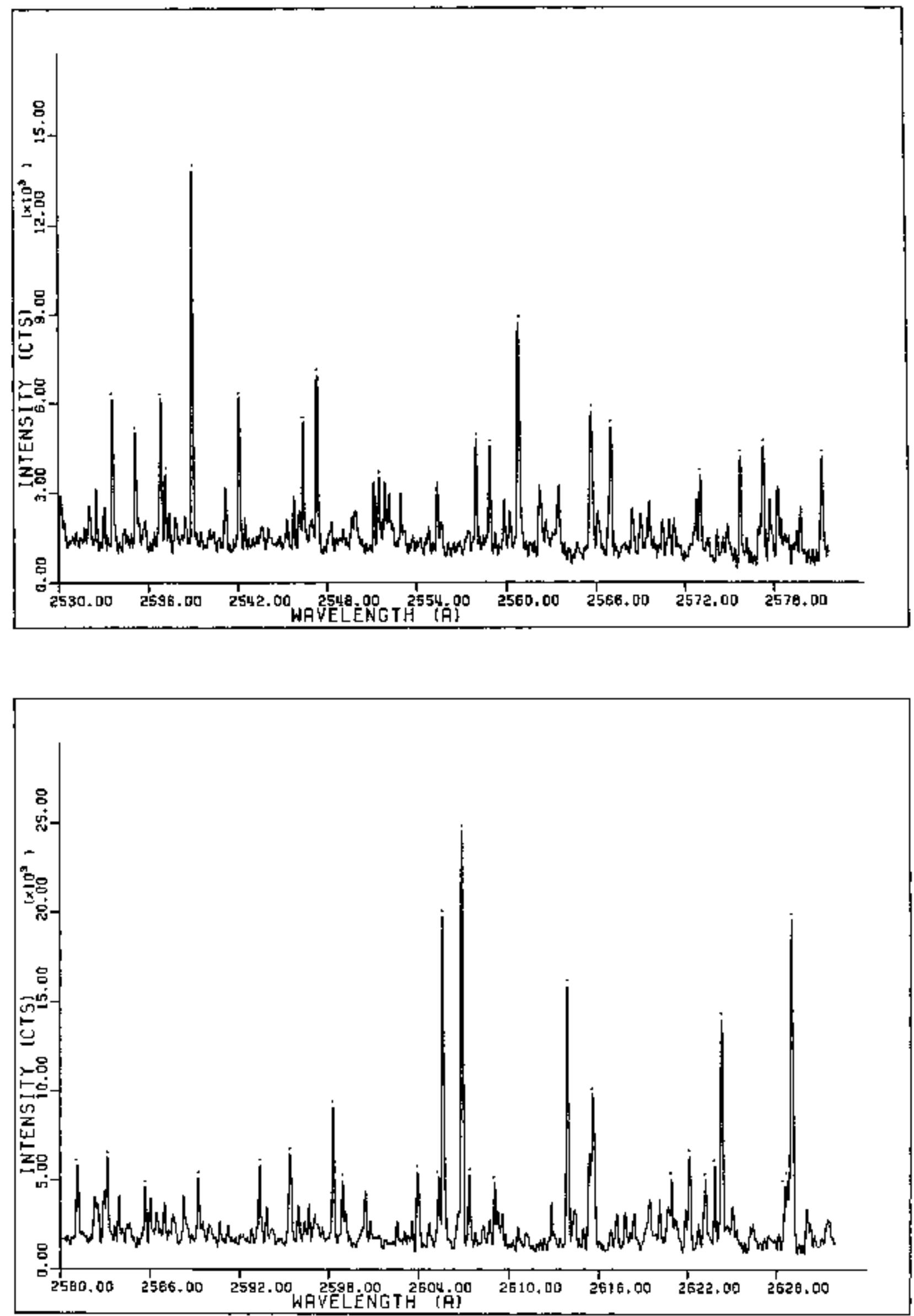


\begin{tabular}{|c|c|}
\hline$\lambda(A)$ & I \\
\hline $\begin{array}{l}2633.2202 \\
2635,6104\end{array}$ & $\begin{array}{l}8263 . \\
6336 .\end{array}$ \\
\hline 2637.3545 & 1611 . \\
\hline 2638.8403 & 12019, \\
\hline $\begin{array}{l}2639.4216 \\
2641.0366\end{array}$ & $\begin{array}{l}7534 . \\
7764 .\end{array}$ \\
\hline 2611.4690 & $9+28$ \\
\hline 2613.1685 & 6732 \\
\hline 2646.2049 & 893. \\
\hline 2647.3030 & 6153 \\
\hline 2651,7603 & $15 \$ 10$. \\
\hline $2652,85 B 6$ & 12908, \\
\hline $\begin{array}{l}2654.9902 \\
2656.7346\end{array}$ & 79438, \\
\hline 2662.6130 & $\begin{array}{l}6500 . \\
6092 .\end{array}$ \\
\hline 2666,6120 & 7149. \\
\hline 2669,7937 & 40111. \\
\hline $267 \pm .592 \mathrm{~s}$ & 12755. \\
\hline $\begin{array}{l}2675,8562 \\
2678.3110\end{array}$ & $\begin{array}{l}9755 . \\
41947 .\end{array}$ \\
\hline 2679.4092 & 21725. \\
\hline 2680,0552 & 6719. \\
\hline $2680+8303$ & $\pi 01$. \\
\hline 2680.5267 & 9328. \\
\hline 2694,2124 & 17233. \\
\hline 2683.9299 & $10 \% 52$. \\
\hline 2691.6709 & 10581. \\
\hline 2693.4937 & PQ42. \\
\hline $\begin{array}{l}2697+3677 \\
2700.9424\end{array}$ & 9634 \\
\hline 2704,4192 & 14764 . \\
\hline 2709,4612 & 16543 , \\
\hline 2740.0562 & 11429 \\
\hline 2711.3323 & 10957, \\
\hline $\begin{array}{l}2712,3518 \\
2714,5146\end{array}$ & $\begin{array}{l}\text { tows. } \\
17647 .\end{array}$ \\
\hline 2715.0864 & 10344 . \\
\hline 2715,4673 & 11268. \\
\hline $27: 6.4187$ & 12934, \\
\hline 2717.2424 & 49496. \\
\hline 2722,1497 & 16935. \\
\hline $727+1294$ & $133 \%$. \\
\hline 2728.4434 & 10231 . \\
\hline 2728.7559 & 10040 \\
\hline
\end{tabular}



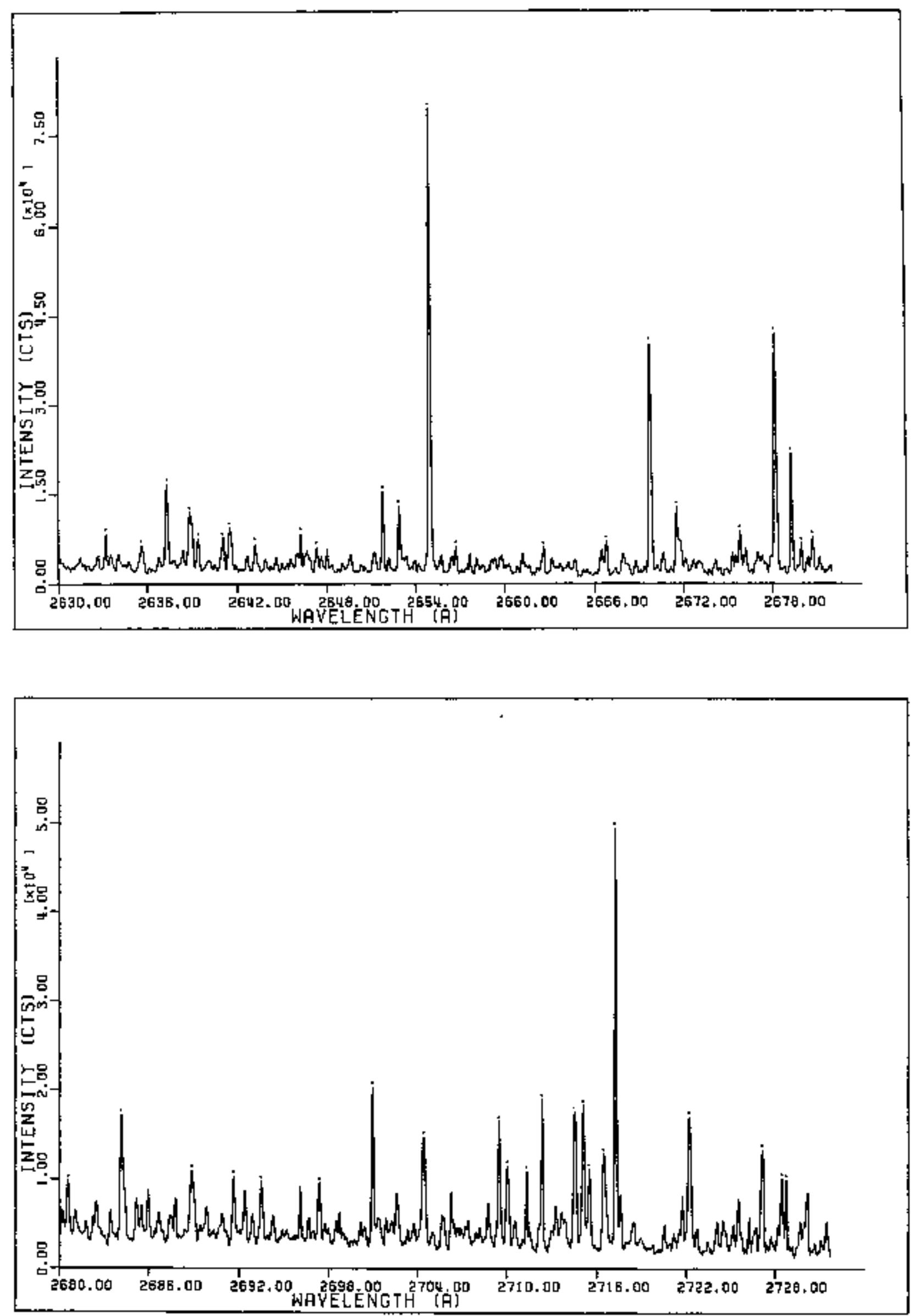


\begin{tabular}{|c|c|}
\hline$\lambda(A)$ & I \\
\hline 270.365 & 10819 \\
\hline $\begin{array}{l}2733.0620 \\
2734.6040\end{array}$ & $\begin{array}{l}16054 . \\
36235 .\end{array}$ \\
\hline 2739.8001 & 15163. \\
\hline $\begin{array}{l}770+1936 \\
274,4146\end{array}$ & 12356. \\
\hline 2748.2879 & $\begin{array}{l}10109 . \\
3056 .\end{array}$ \\
\hline $\begin{array}{l}2748.8674 \\
2750.7307\end{array}$ & $\begin{array}{l}3602 . \\
9046 .\end{array}$ \\
\hline 2751.6341 & 10850. \\
\hline $\begin{array}{l}2 \% 4.0076 \\
2755.0064\end{array}$ & 13561 \\
\hline $\begin{array}{l}2755+8064 \\
7759,60355\end{array}$ & $\begin{array}{l}11417 \\
10450\end{array}$ \\
\hline 2758.8904 & 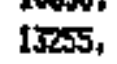 \\
\hline 2760.6252 & 15261 \\
\hline 2762,9392 & 11175 \\
\hline 2771.2266 & 12056 \\
\hline 2773.0998 & 26631 \\
\hline 2774 , 日8:7 & 13759 \\
\hline $\begin{array}{l}277.1375 \\
2780.9282\end{array}$ & $\begin{array}{l}\text { 11608, } \\
\text { 1024!. }\end{array}$ \\
\hline 2700.7093 & 12486, \\
\hline 2783.2783 & 12509 \\
\hline $2705.327 \%$ & 42116 \\
\hline 2787.1653 & A1B12. \\
\hline 2769,1709 & \pm 1340 \\
\hline 2790.0037 & 18363 \\
\hline $2 \pi 0.6410$ & 15181 \\
\hline 2792,5015 & 17131 \\
\hline 2796.0242 & 11641 \\
\hline 2796,6648 & 13753 \\
\hline 2798,1379 & 13430 \\
\hline 2796.7783 & 3704 . \\
\hline $\begin{array}{l}2000.7000 \\
2003,5623\end{array}$ & $47623+$ \\
\hline $2003.5 E 23$ & $\begin{array}{l}1395 \\
11158\end{array}$ \\
\hline 2816,9045 & 20152 , \\
\hline 2824,3633 & 10589. \\
\hline 2822.3469 & $120 / 2$ \\
\hline $\begin{array}{l}2624,0142 \\
2925,2312\end{array}$ & 13196. \\
\hline
\end{tabular}



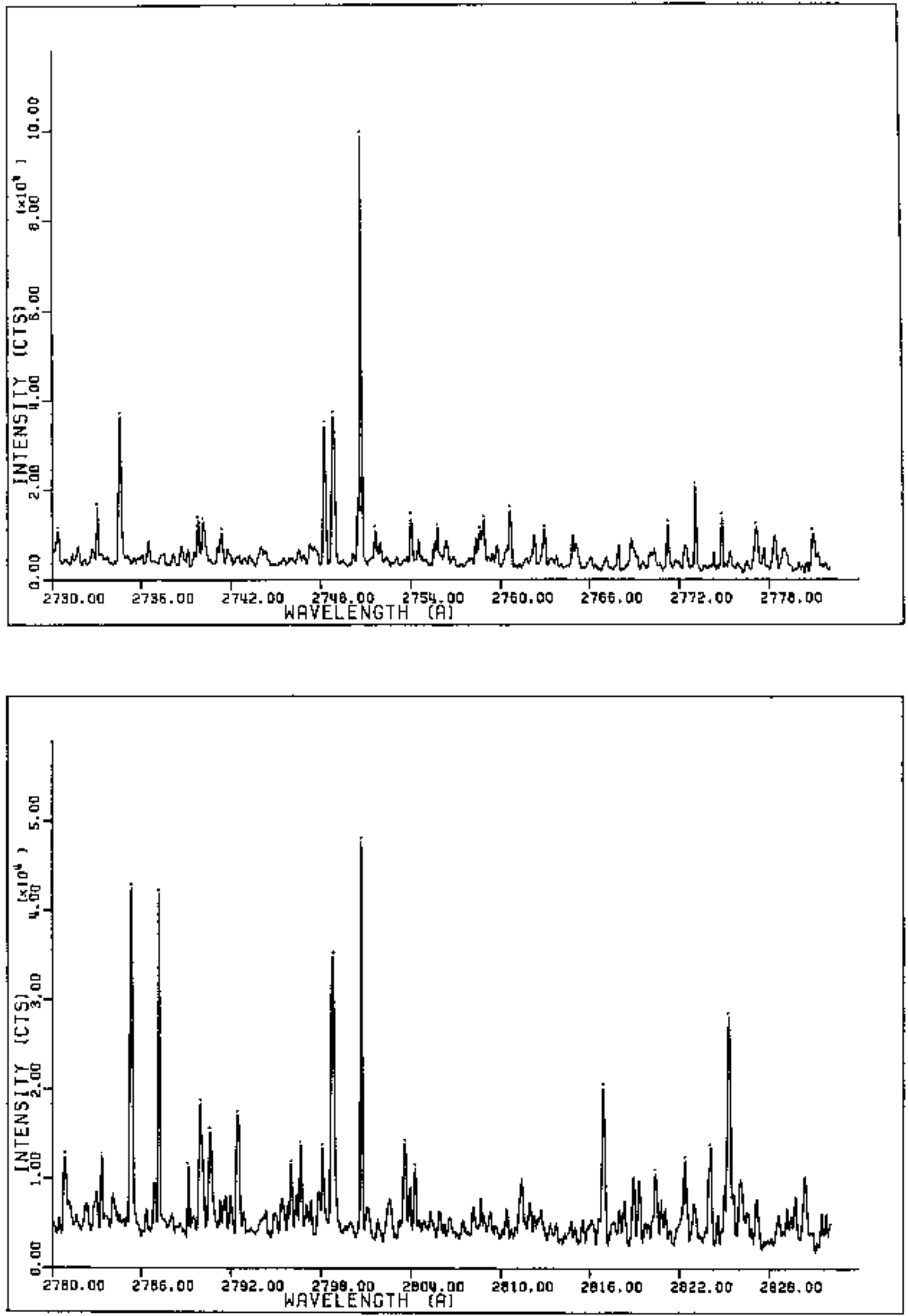


\begin{tabular}{|c|c|}
\hline$\lambda(\mathrm{A})$ & I \\
\hline 2830.1172 & 1324 \\
\hline 2833,5012 & 18 \\
\hline 2638.0347 & 1283 \\
\hline 2841,1633 & 3915 \\
\hline $2 B 1.6741$ & $M$ \\
\hline 2643.9727 & 35 \\
\hline $284+2920$ & 1783 \\
\hline 2947,6677 & 17236 \\
\hline 2652,6562 & 1334 \\
\hline 2053.6140 & 20042 \\
\hline 2659.6799 & 29317 \\
\hline 2660.6377 & 1446 \\
\hline 201,4038 & 11893 \\
\hline 2661.7231 & 13151 \\
\hline 2863,440 & 13545 \\
\hline 2066.0649 & 62909 \\
\hline 2367.0227 & 17147 \\
\hline 2867.6611 & 21257 \\
\hline 2869.440 & 17516 \\
\hline 2870.1705 & 26685 \\
\hline 2873.4675 & 14793 \\
\hline 2974.0461 & 1329 \\
\hline 2874.6203 & 1173 \\
\hline 2677.8132 & 12922 \\
\hline 2085,5065 & 2087 \\
\hline 2834.1938 & 23619 \\
\hline $2897+8149$ & 52929 \\
\hline 2009.2163 & 22105 \\
\hline 2681 , 065 & 20975 \\
\hline 2993.7390 & 49523 \\
\hline $\begin{array}{l}2998.9624 \\
2899.9177\end{array}$ & $\begin{array}{l}3186 \\
29129\end{array}$ \\
\hline 290.6187 & 3001 \\
\hline 2901.0007 & 53528 \\
\hline 2902.7944 & 35815 \\
\hline 2904.2495 & 76003 \\
\hline 2904.5679 & 8112. \\
\hline $2 \% 05.5672$ & 22699 \\
\hline 2913,2224 & 6627 \\
\hline 2916.6709 & 23617 \\
\hline 2918,6458 & 25304 \\
\hline 2920,4292 & 23511 \\
\hline $2923,0+10$ & 20668 \\
\hline $\begin{array}{l}2925.2069 \\
2926.7993\end{array}$ & $\begin{array}{l}58442 \\
36120\end{array}$ \\
\hline $2927+5000$ & 31597 , \\
\hline 2928,9651 & 35887 \\
\hline 2929,4109 & 7561 \\
\hline 2930.1733 & 20714 \\
\hline 2931.3957 & BN247 \\
\hline
\end{tabular}



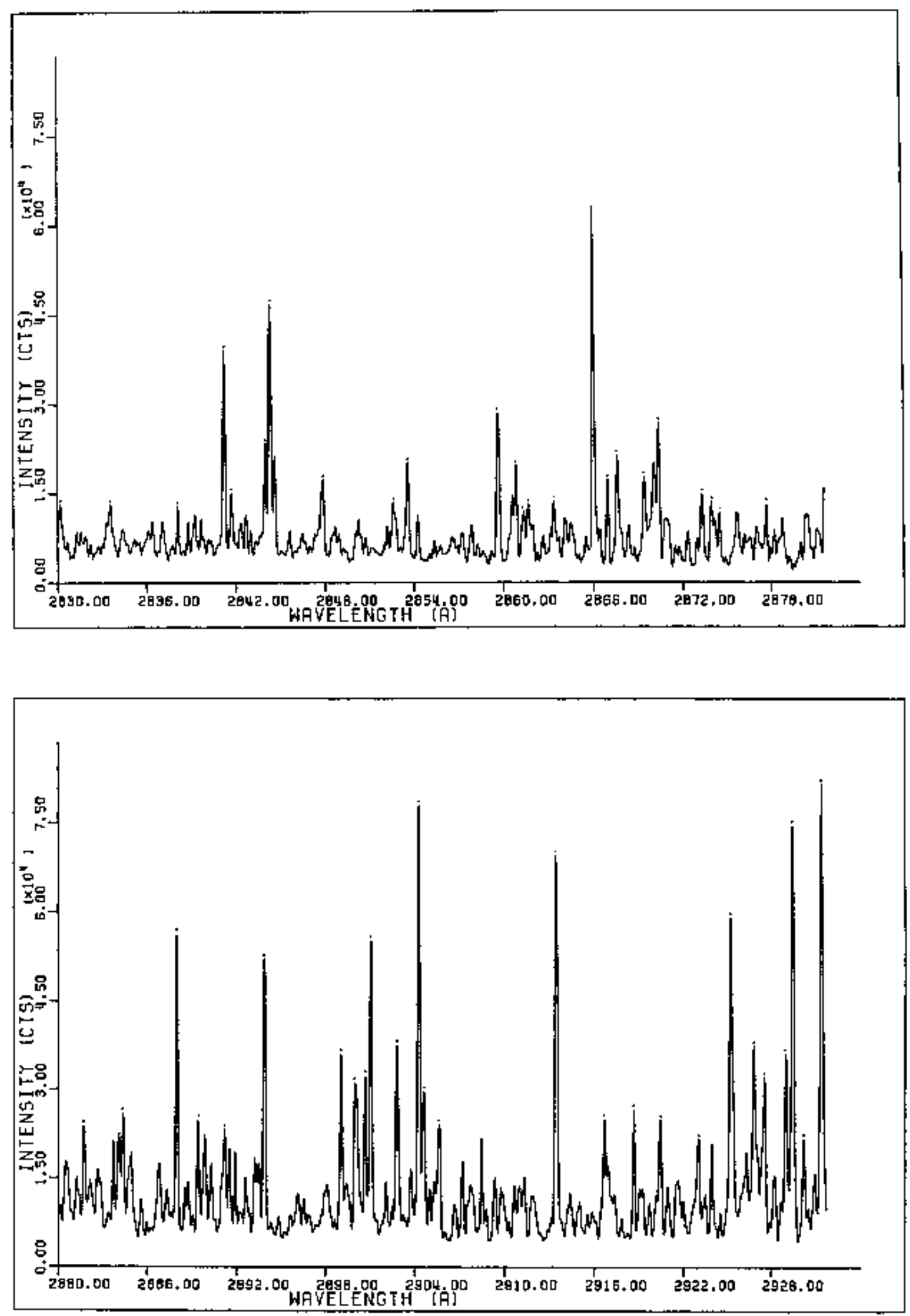


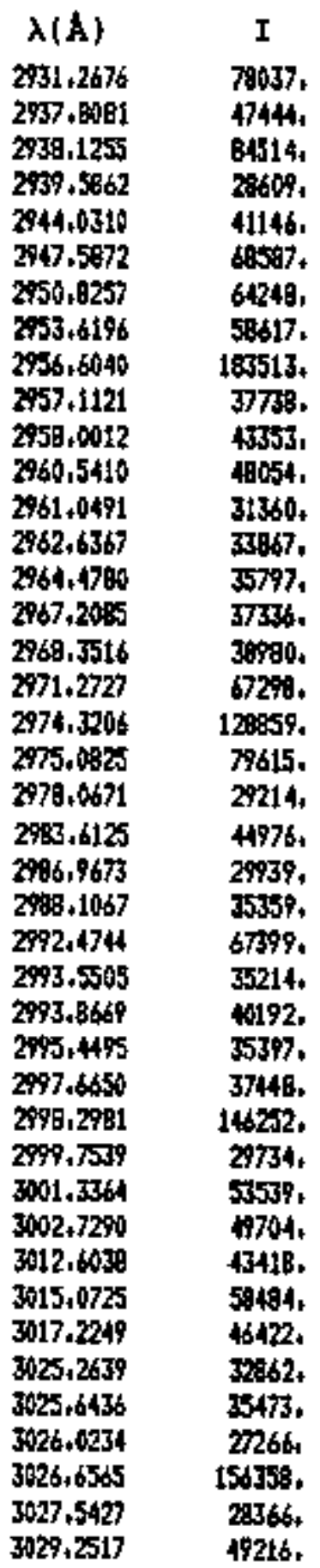



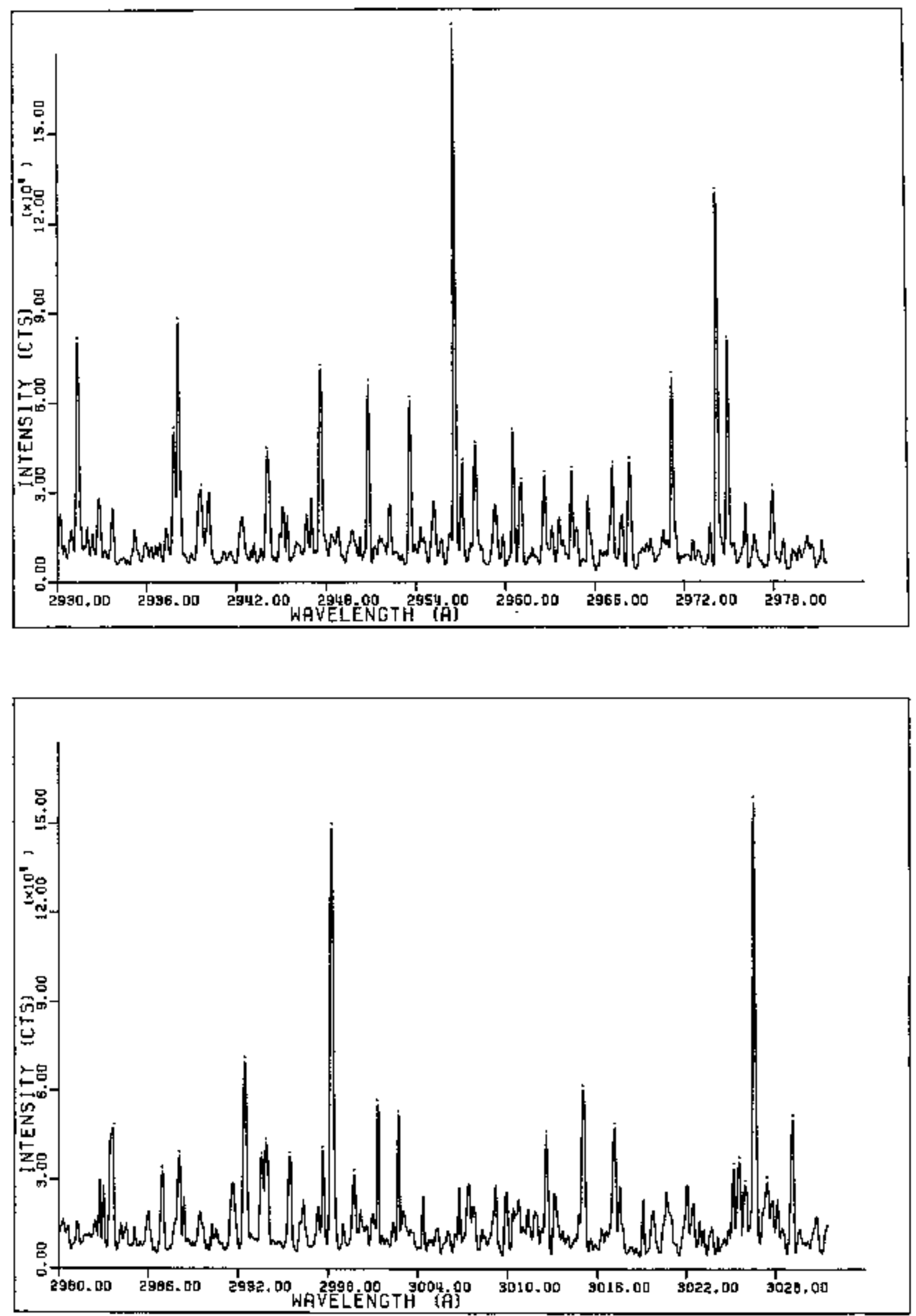


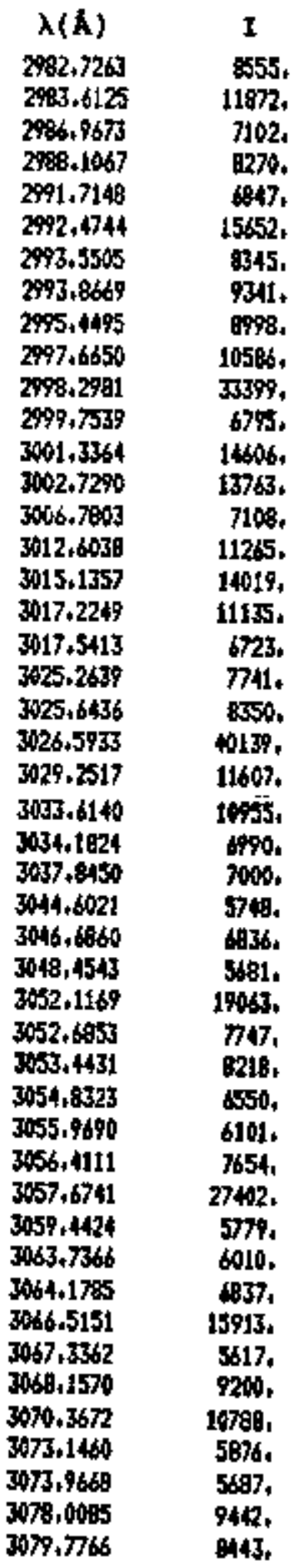



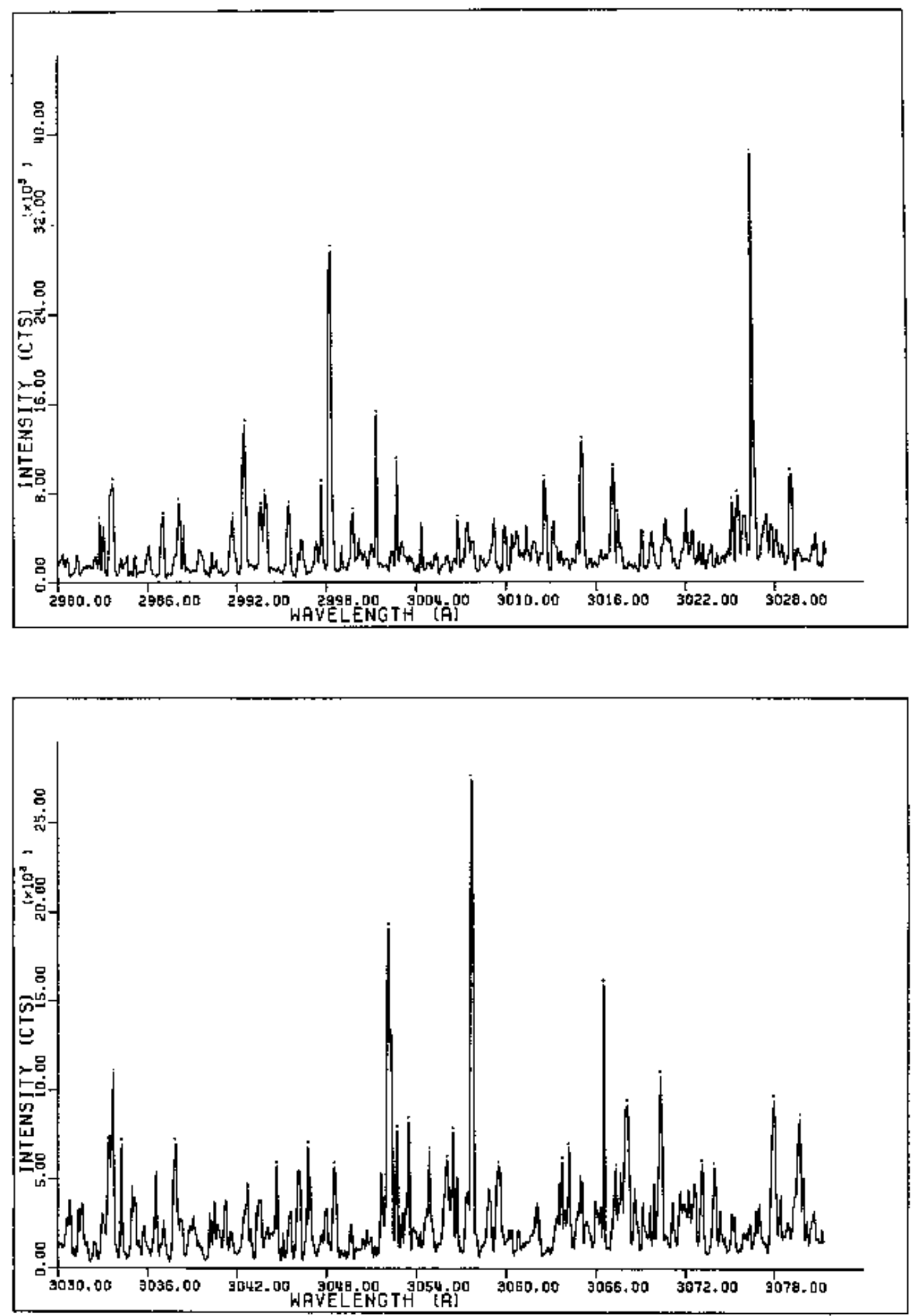


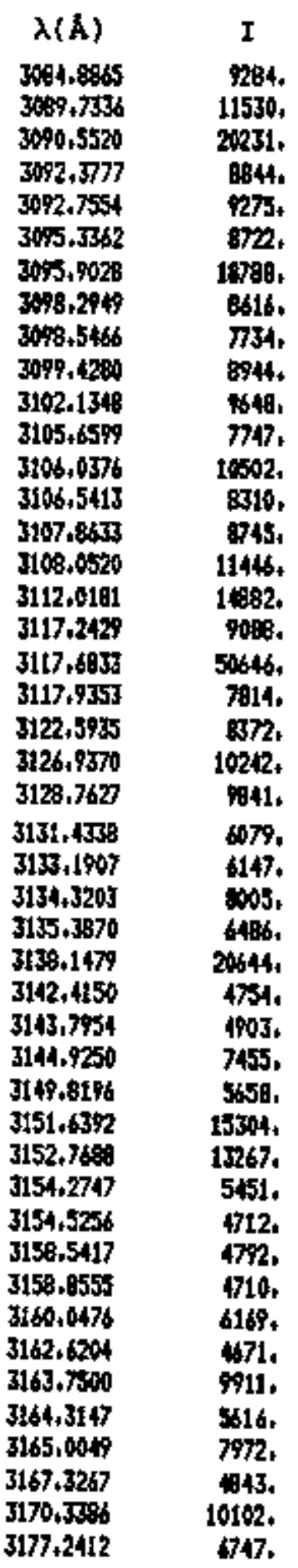



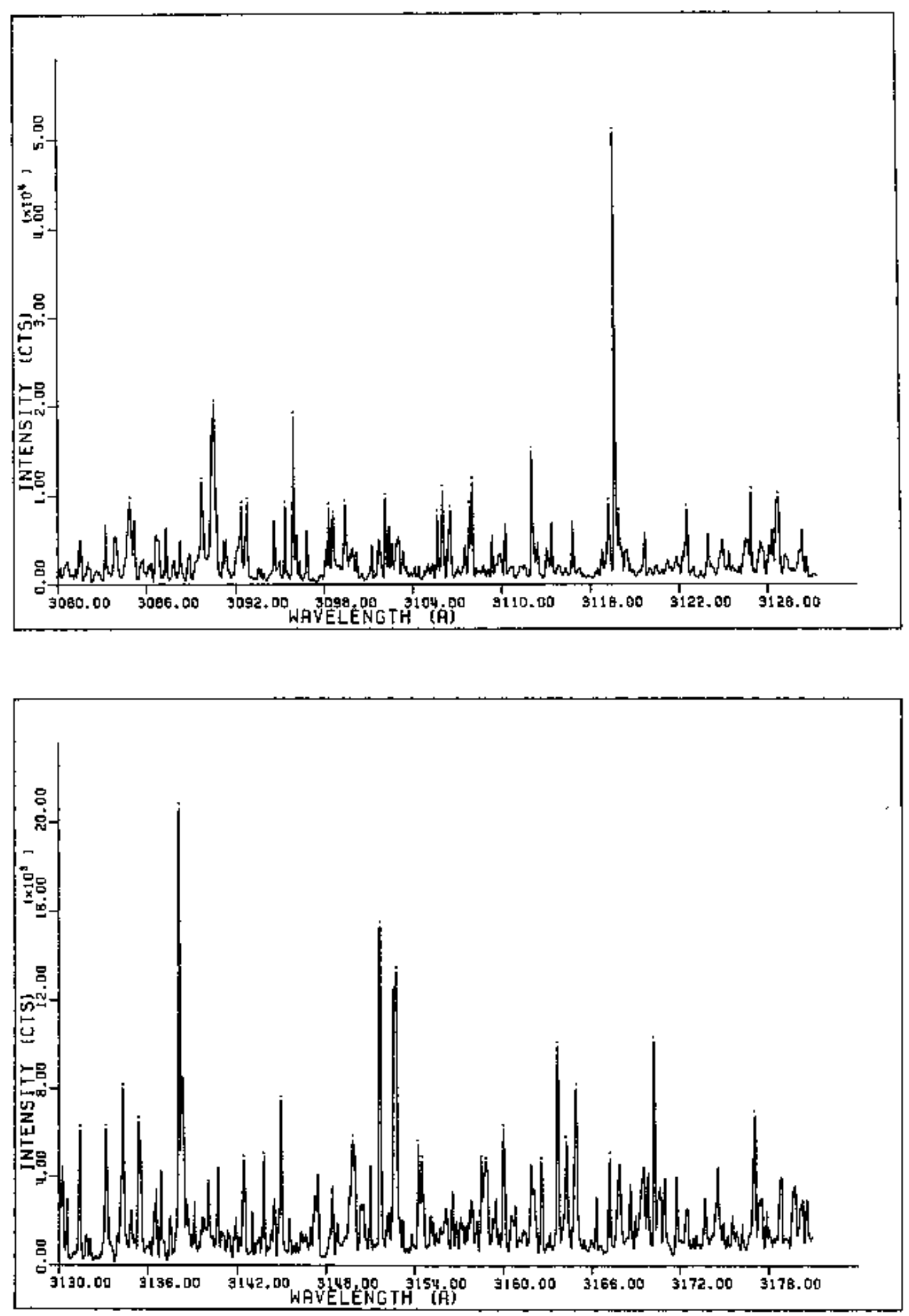


\begin{tabular}{|c|c|}
\hline$\lambda(\mathbf{A})$ & $\mathbf{I}$ \\
\hline 3185.3010 & 11726 \\
\hline 3685.9269 & 23825. \\
\hline 3187.6162 & 7694 \\
\hline 3189.1179 & 762 \\
\hline $3189+4009$ & 7264. \\
\hline 3193,9358 & $\$ 191$. \\
\hline 3184,9368 & 6255, \\
\hline 3196,8140 & 419, \\
\hline $3\{97,1267$ & 42077 . \\
\hline 3200,8811 & $7106+$ \\
\hline $3203+5090$ & 1347 \\
\hline 3204,3850 & 1214. \\
\hline 3200.5771 & 5126. \\
\hline $3240+9526$ & 5998, \\
\hline 3212,2061 & \$5.5. \\
\hline $\begin{array}{l}3223.2673 \\
3215.3347\end{array}$ & $\begin{array}{l}1418 . \\
1676 .\end{array}$ \\
\hline 3215.8977 & 502, \\
\hline 3223,4063 & 11680 . \\
\hline 3224.3447 & 1232, \\
\hline 325,8464 & 16573. \\
\hline 3234,1011 & 7508. \\
\hline 3230.1204 & 432, \\
\hline 3235.9558 & 3964 \\
\hline 5737,3633 & 5004 \\
\hline $3239,120 \mathrm{~B}$ & 3426, \\
\hline $3241,47 \%$ & 8101. \\
\hline 3244.0937 & $\$ 592$ \\
\hline 3246.3764 & 8059 \\
\hline 3252,4513 & 5791 \\
\hline 3552.7044 & 3912 \\
\hline $3255+244$ & 10602 . \\
\hline 3255.5532 & 3447 \\
\hline $3256+6685$ & $6 \%$ \\
\hline 3259,6901 & 4653 \\
\hline 3261.3149 & 574 \\
\hline 3263.1729 & 6158 \\
\hline 3264,8450 & 3925 \\
\hline 3265.5879 & 5493 \\
\hline 3266,2682 & 371 \\
\hline 3268,9312 & 6596 \\
\hline 3271,5306 & 7196 \\
\hline 3275.6765 & 4128 \\
\hline $3276+9750$ & 3474 \\
\hline 3279.8973 & \\
\hline
\end{tabular}



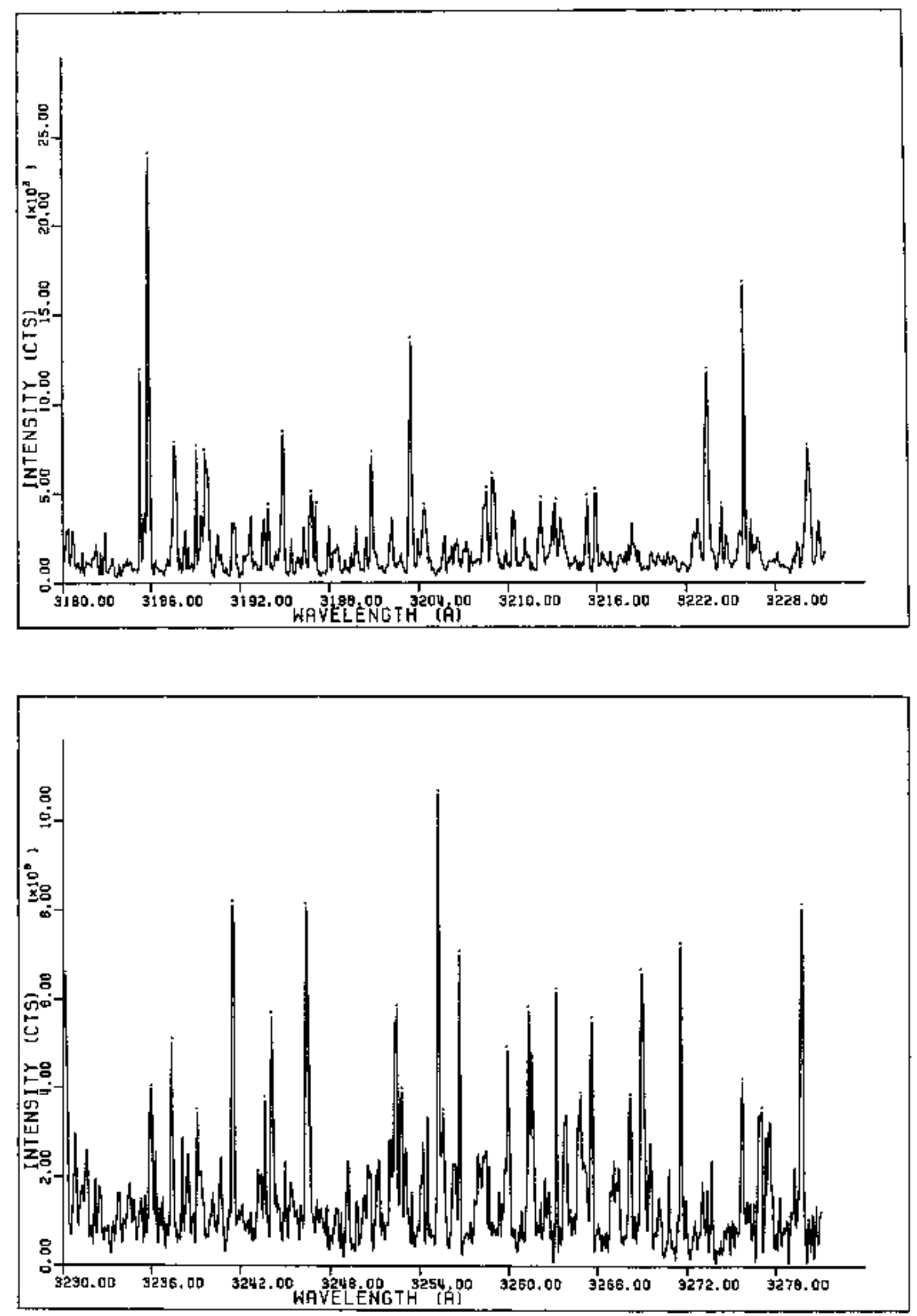


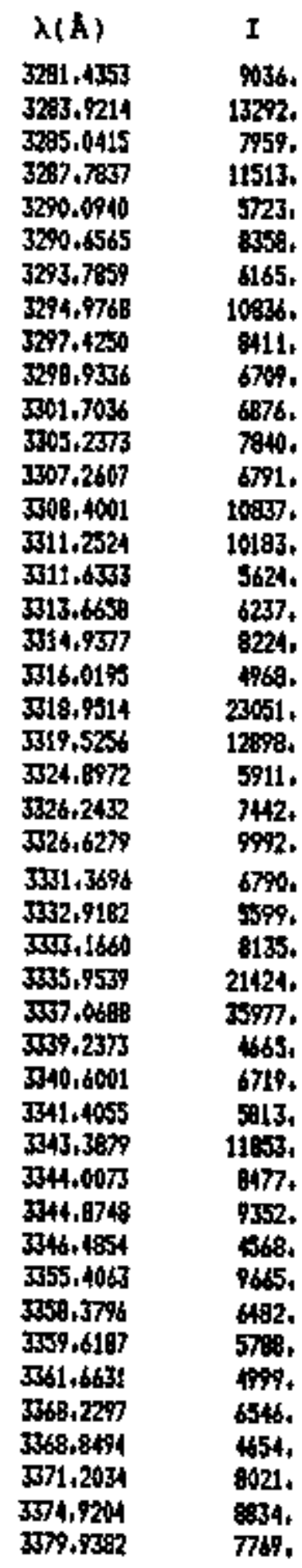



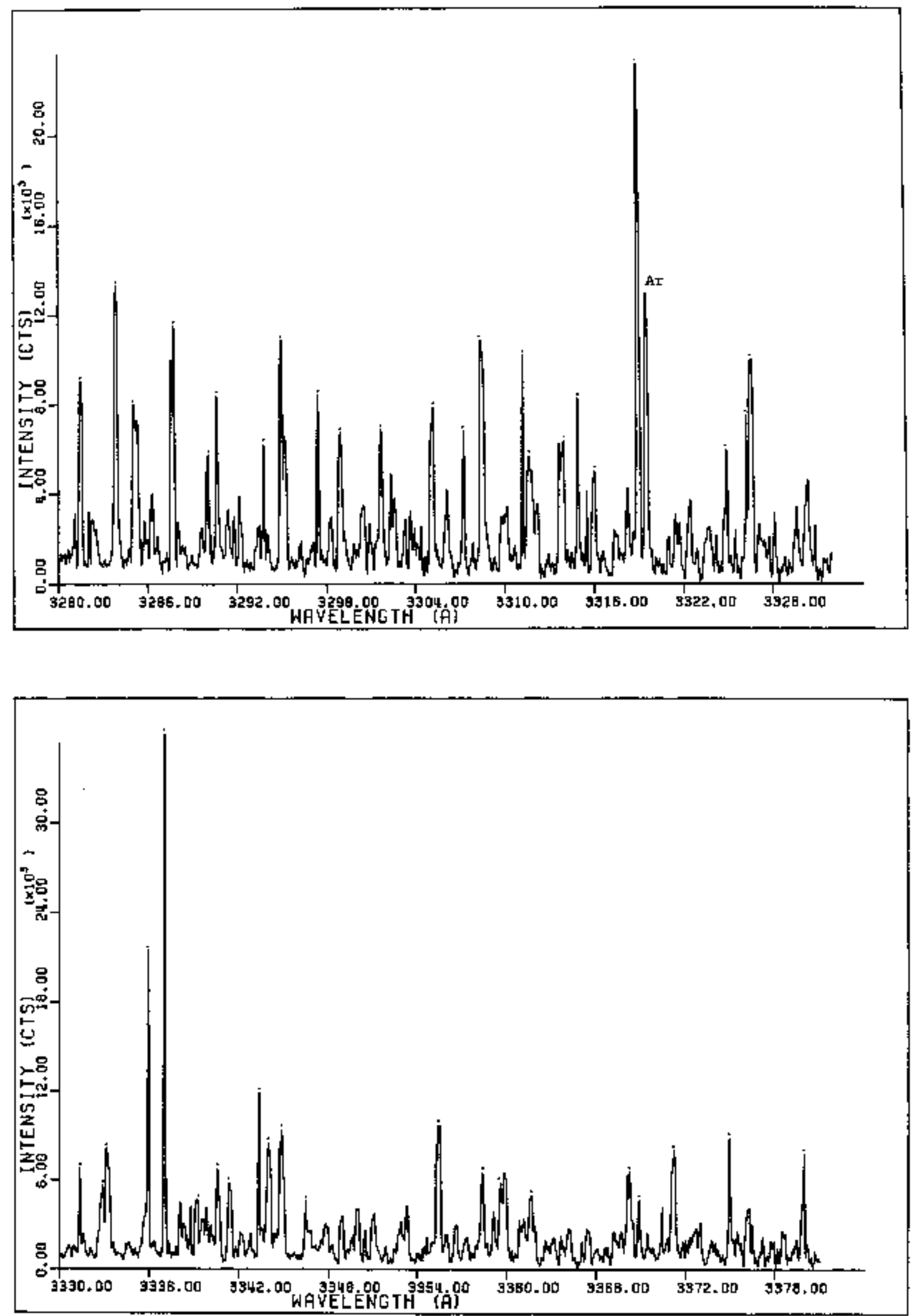


\begin{tabular}{|c|c|}
\hline$\lambda(A)$ & $I$ \\
\hline $\begin{array}{l}3333.5142 \\
3396,0461 \\
3399.6992\end{array}$ & $\begin{array}{r}7599 \\
5435\end{array}$ \\
\hline 3389.6992 & 10962 \\
\hline $\begin{array}{l}391,3564 \\
3392,4063\end{array}$ & $\begin{array}{l}4352 \\
5622\end{array}$ \\
\hline 3593.3525 & 19524 \\
\hline 3402,6567 & 59115 \\
\hline $303.45 \%$ & 1466 \\
\hline 3404,632 & 10263. \\
\hline 3407,3499 & $\mathbf{4 5 3 8}$ \\
\hline 308.4612 & 9063 \\
\hline 3409.5728 & 5060 \\
\hline 3411,1762 & 1107 \\
\hline $\begin{array}{l}3412,4751 \\
3415.9331\end{array}$ & $\begin{array}{l}7849 \\
449\end{array}$ \\
\hline 316.1182 & An12 \\
\hline $3416+6741$ & 6404 \\
\hline $3,17,0444$ & 12033. \\
\hline 317,4768 & 6222 \\
\hline $3+17,8472$ & 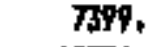 \\
\hline 326,0601 & 10354 \\
\hline $\begin{array}{l}3420,2369 \\
3429,6416\end{array}$ & $\begin{array}{l}\$ B 46, \\
4505 .\end{array}$ \\
\hline 345.0701 & 5559 \\
\hline 3433.6936 & 795 \\
\hline 3434.3706 & 5104 \\
\hline $\begin{array}{l}3134,6169 \\
3436,7712\end{array}$ & $\begin{array}{l}6219 \\
5902\end{array}$ \\
\hline $3437+1+04$ & 6320 \\
\hline 369,4177 & 6087 \\
\hline 3440.2100 & 564 \\
\hline 341.6951 & 11749 \\
\hline $\begin{array}{l}346,1267 \\
3446,4346\end{array}$ & $\begin{array}{l}5176 \\
5648\end{array}$ \\
\hline 3450,4969 & 5023 \\
\hline 3453.2666 & $2875 \%$ \\
\hline $\begin{array}{l}3455.3591 \\
3459.5477\end{array}$ & 5647 \\
\hline 365, 0994 & 969. \\
\hline 3464.5994 & 6730 \\
\hline 3466,4362 & 4993. \\
\hline $\begin{array}{l}3470.7468 \\
3471.4691\end{array}$ & $90 \%$. \\
\hline $\begin{array}{l}3471,4654 \\
347,0080\end{array}$ & $\begin{array}{l}1724, \\
6742\end{array}$ \\
\hline & 60 \\
\hline & 690 \\
\hline
\end{tabular}



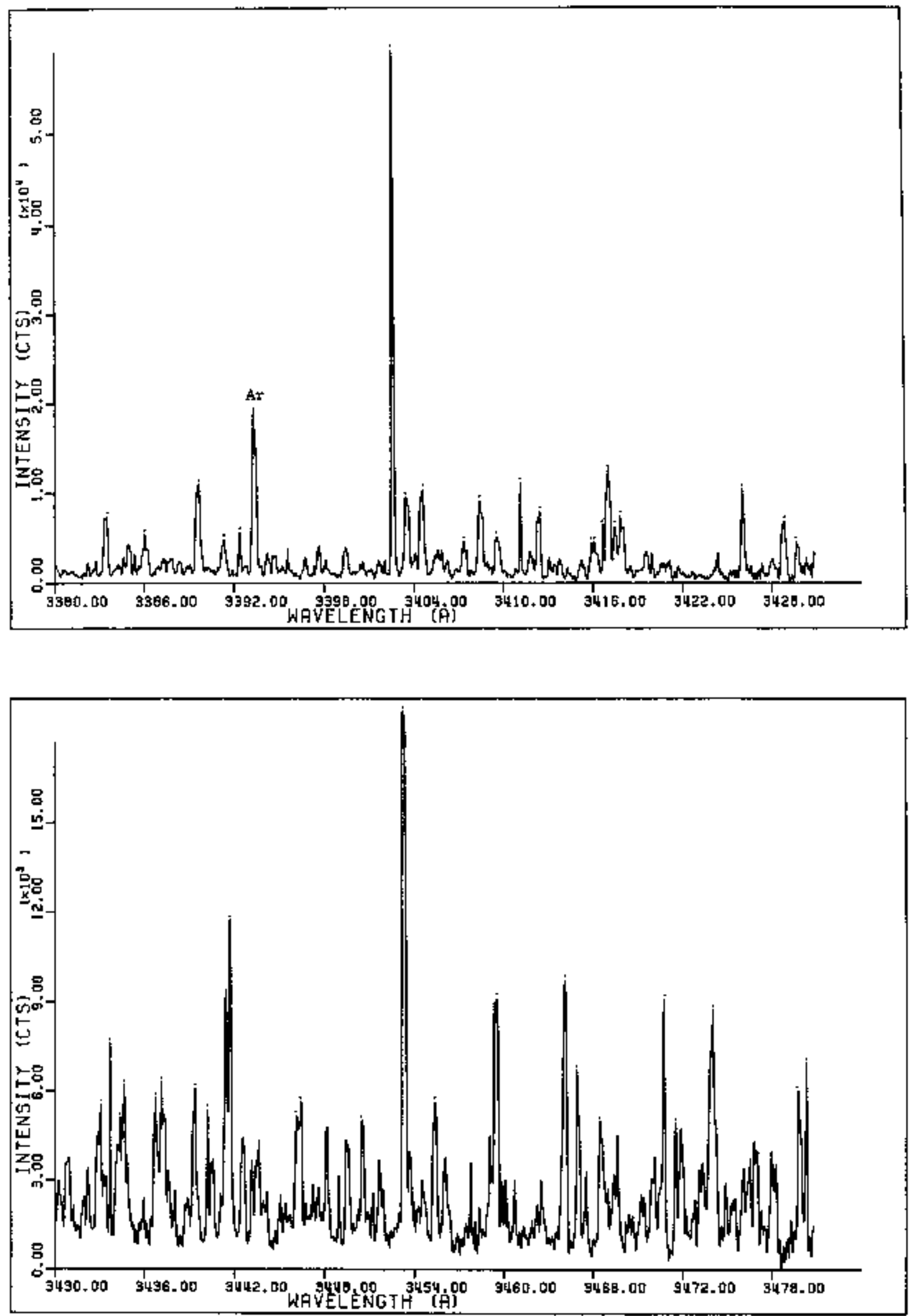


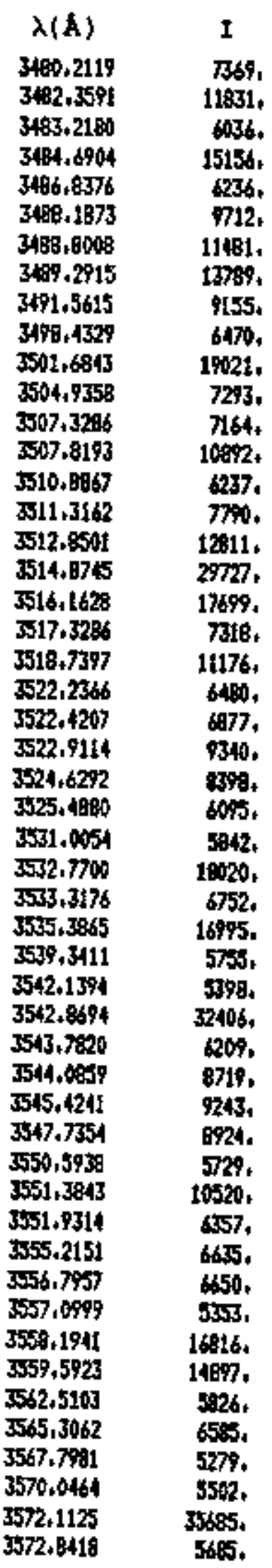



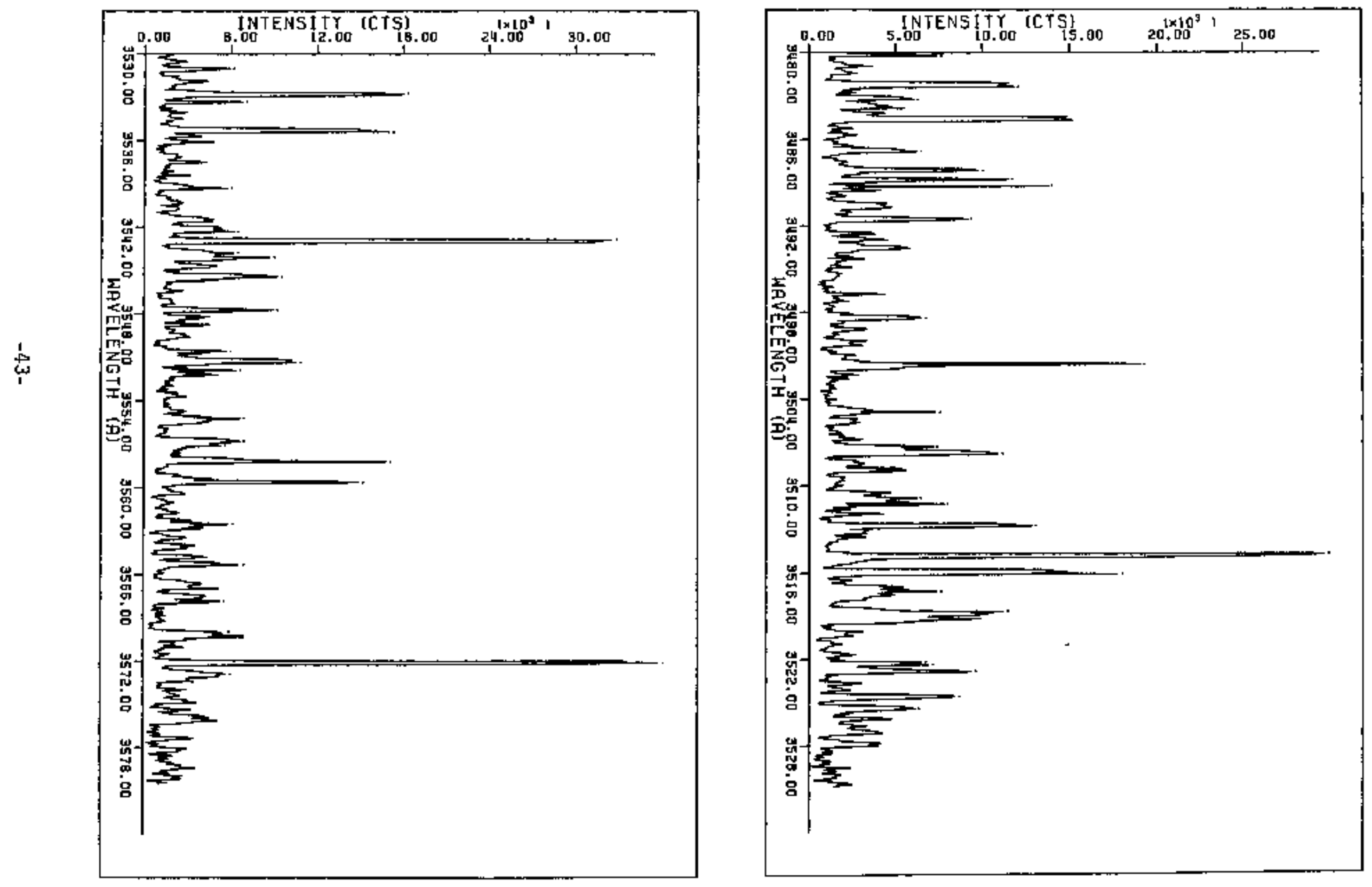


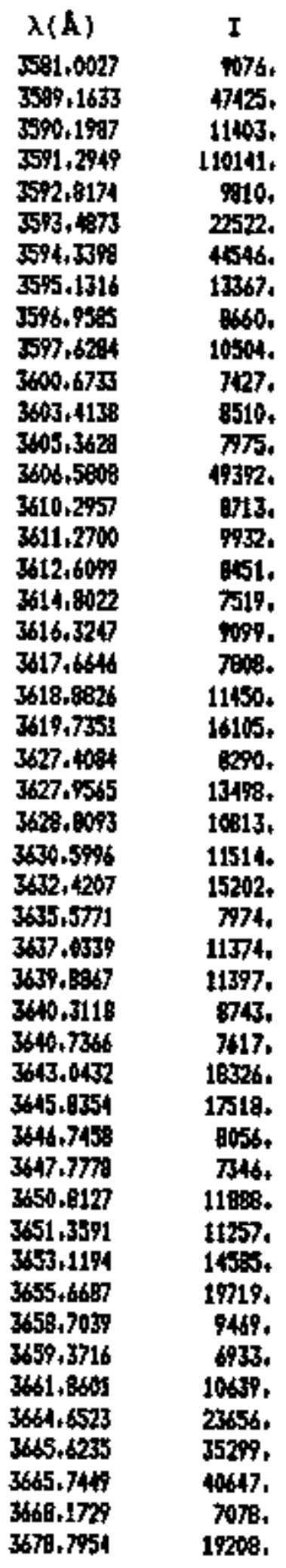



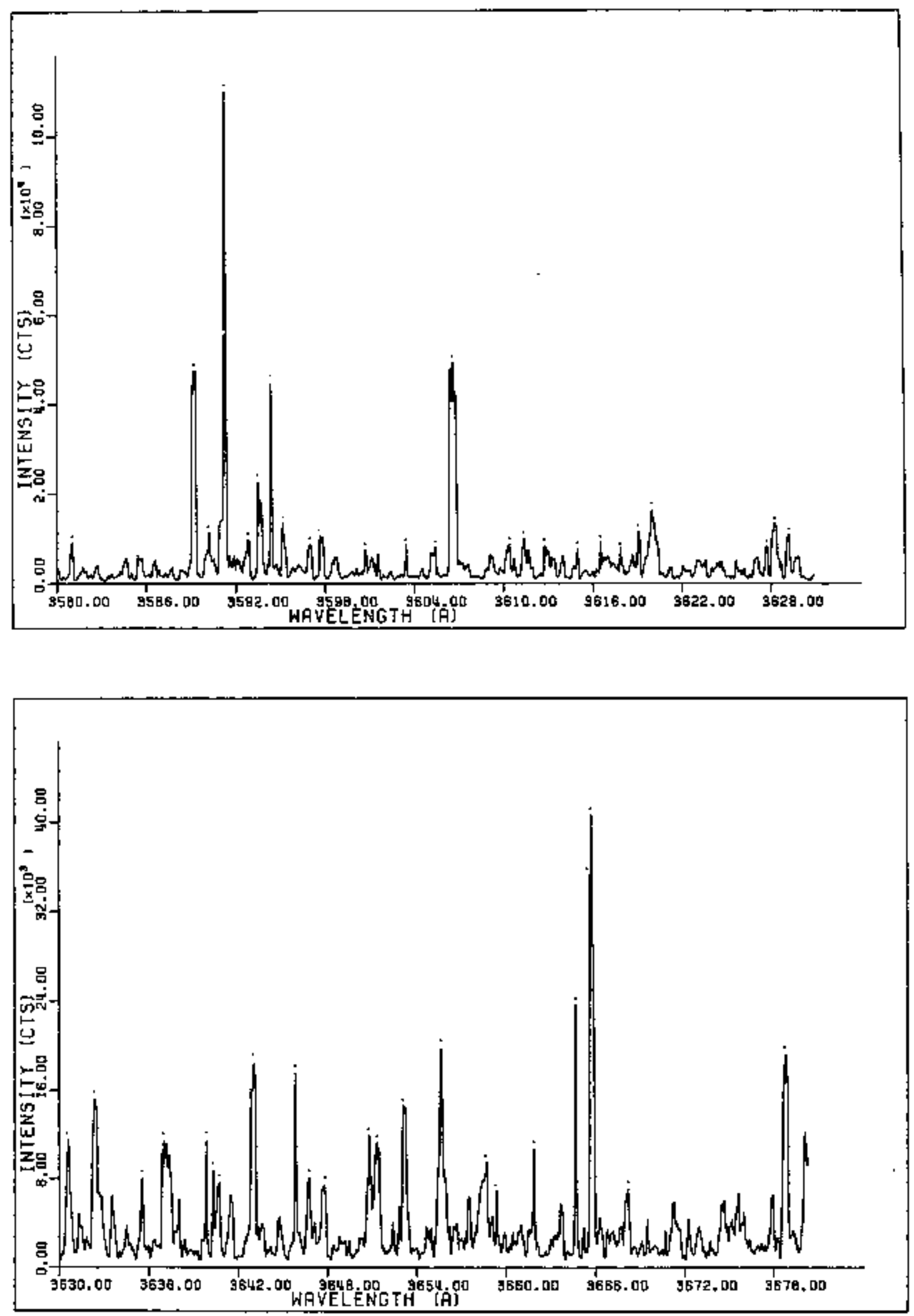


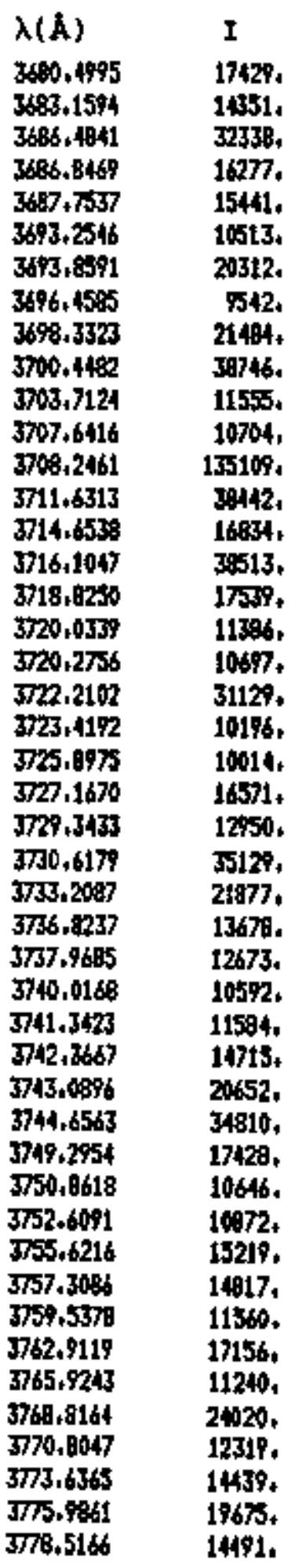



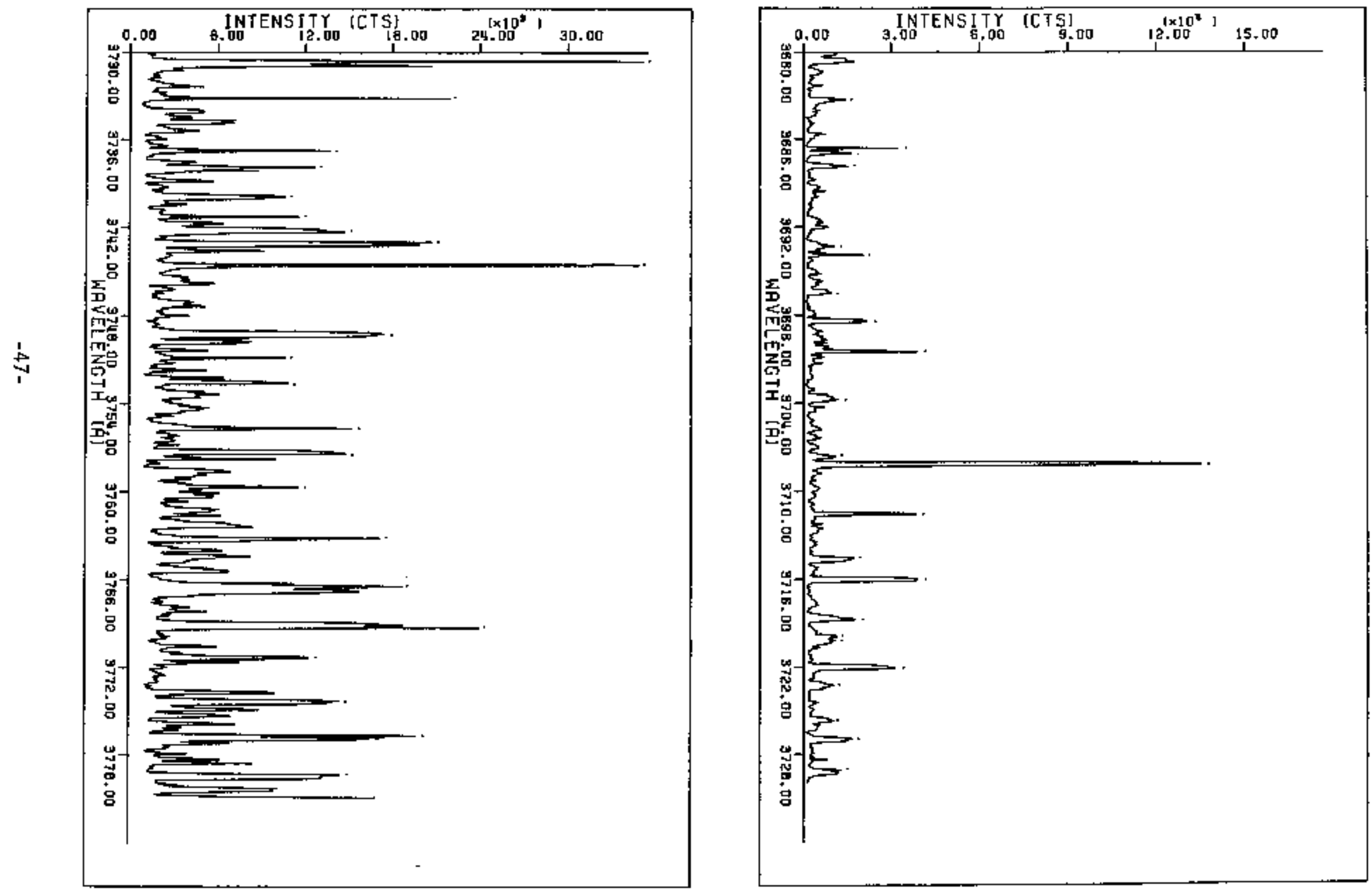


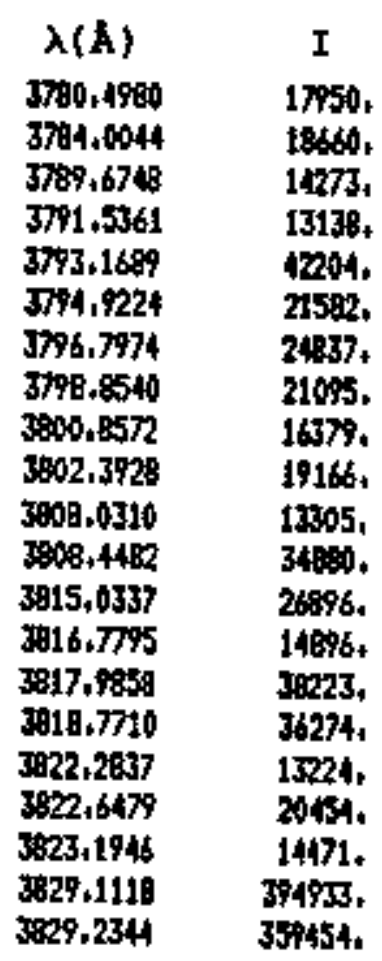



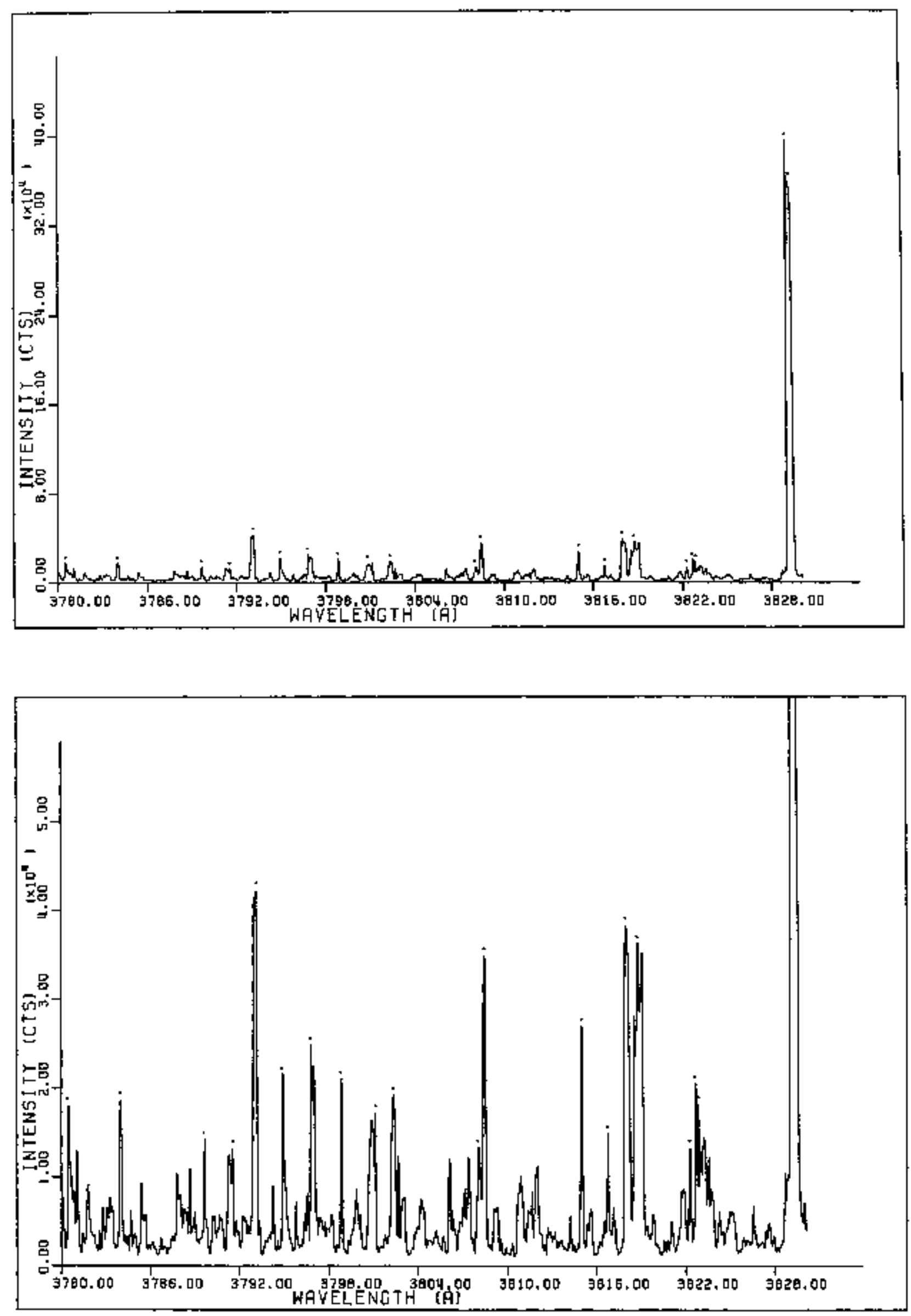


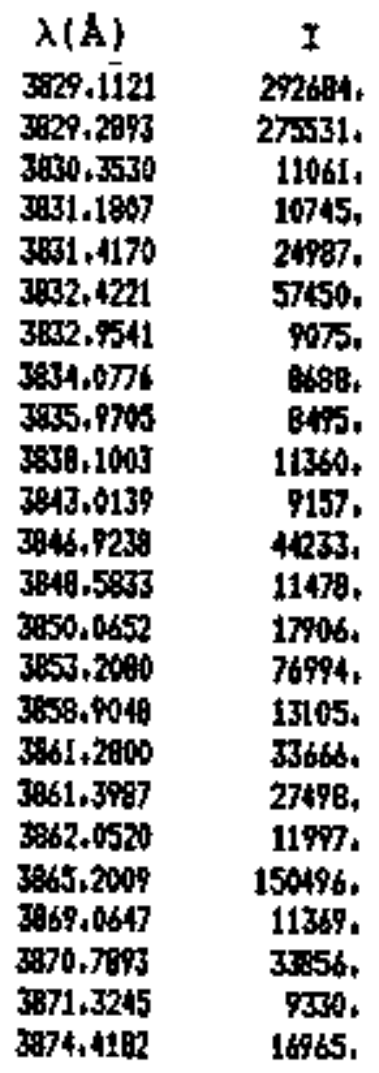



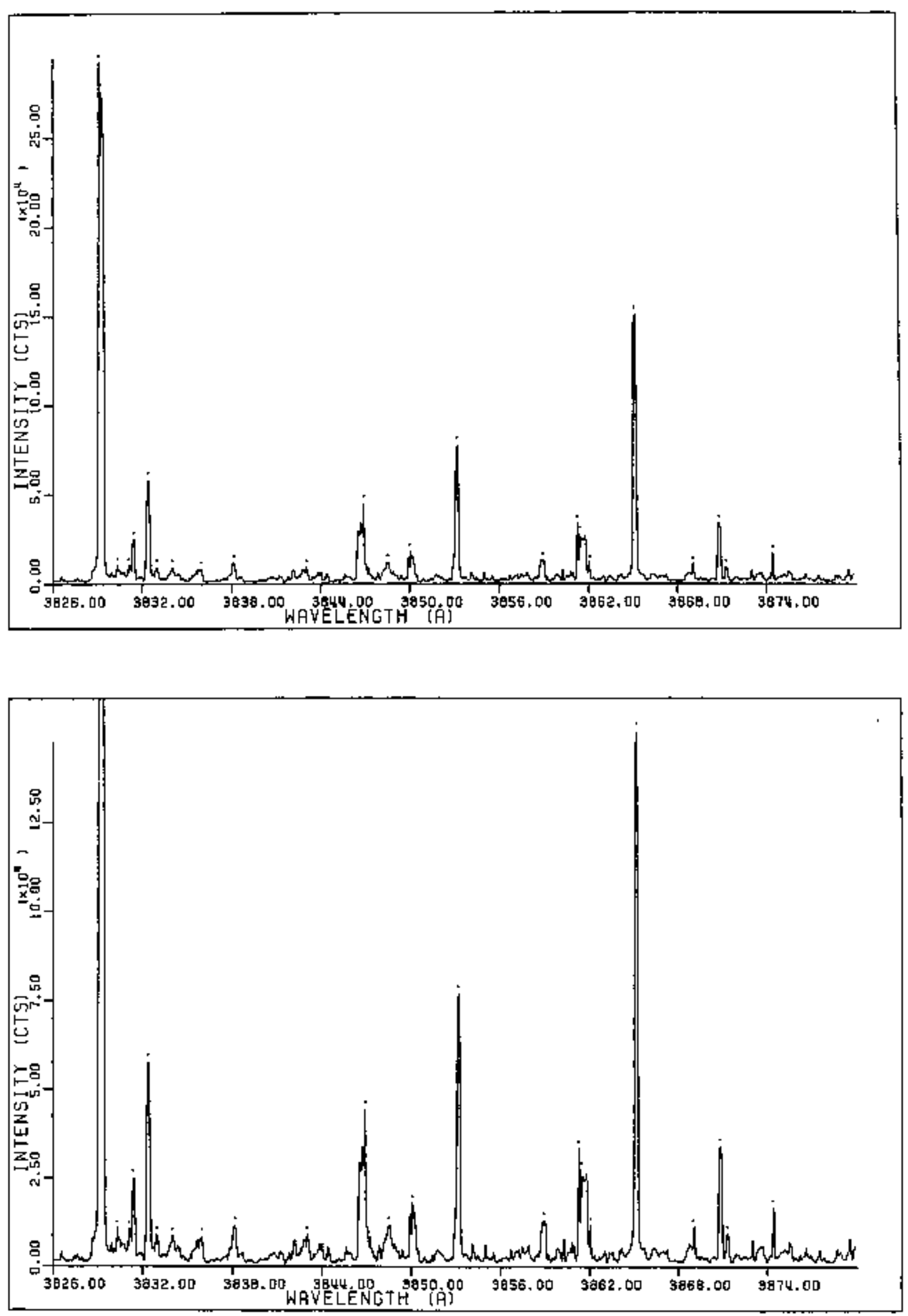


\begin{tabular}{|c|c|}
\hline$\lambda(A)$ & I \\
\hline $\begin{array}{l}3892.7944 \\
3892,9136\end{array}$ & $\begin{array}{l}76503 \\
6965 .\end{array}$ \\
\hline $3887 \cdot 4392$ & P30s. \\
\hline 3899.1539 & 87497. \\
\hline 3991 , 3101 & 28518 \\
\hline 3991,9651 & $24123+$ \\
\hline 7992,7393 & 10725. \\
\hline 3094.7013 & 11469, \\
\hline $3 \% 6,4314$ & 16866. \\
\hline 3902,2076 & \$1710. \\
\hline 3504,815 & 13094, \\
\hline 396.1975 & 16303 \\
\hline 3909.3538 & 1774 \\
\hline 3910.6042 & $50 \%$ \\
\hline 3913,1649 & 18726. \\
\hline 3910.4053 & 11099 \\
\hline 3718.5810 & 1153\%. \\
\hline 3918.7625 & $\$ 321$. \\
\hline 3719.8940 & 14407. \\
\hline 392,9907 & 18741. \\
\hline $\begin{array}{l}3923,7053 \\
3927,1592\end{array}$ & $\begin{array}{l}17445, \\
14276,\end{array}$ \\
\hline 3932.0564 & 20635, \\
\hline 3932,5901 & 11242, \\
\hline 3933,6575 & 10964. \\
\hline 3935,6145 & 20255. \\
\hline 3736.2660 & 11222, \\
\hline $397+0969$ & 18975. \\
\hline 396.0624 & 13169. \\
\hline 3941.0107 & 11013. \\
\hline 3941.260 & 19192. \\
\hline $\begin{array}{l}3946.2386 \\
3949.7197\end{array}$ & $\begin{array}{l}1420 \% \\
24109 .\end{array}$ \\
\hline 3949.5094 & 16304 . \\
\hline 3950,9138 & 53637 , \\
\hline $\begin{array}{l}3954,2939 \\
3959,3345\end{array}$ & 26168. \\
\hline $\begin{array}{l}3939.3315 \\
3 \% 63.8413\end{array}$ & $\begin{array}{l}\text { 15its. } \\
17951 .\end{array}$ \\
\hline $\begin{array}{l}395 \\
7766,45044\end{array}$ & 13215 \\
\hline $\begin{array}{l}3866,4504 \\
3 \% 67,4589\end{array}$ & $12476+$ \\
\hline $\begin{array}{l}3967,4585 \\
3967,6957\end{array}$ & $\begin{array}{l}33535, \\
12013 .\end{array}$ \\
\hline 3968,0515 & 15610 , \\
\hline $\begin{array}{l}3778.4290 \\
3978.6069\end{array}$ & $41738+$ \\
\hline & 46243. \\
\hline
\end{tabular}



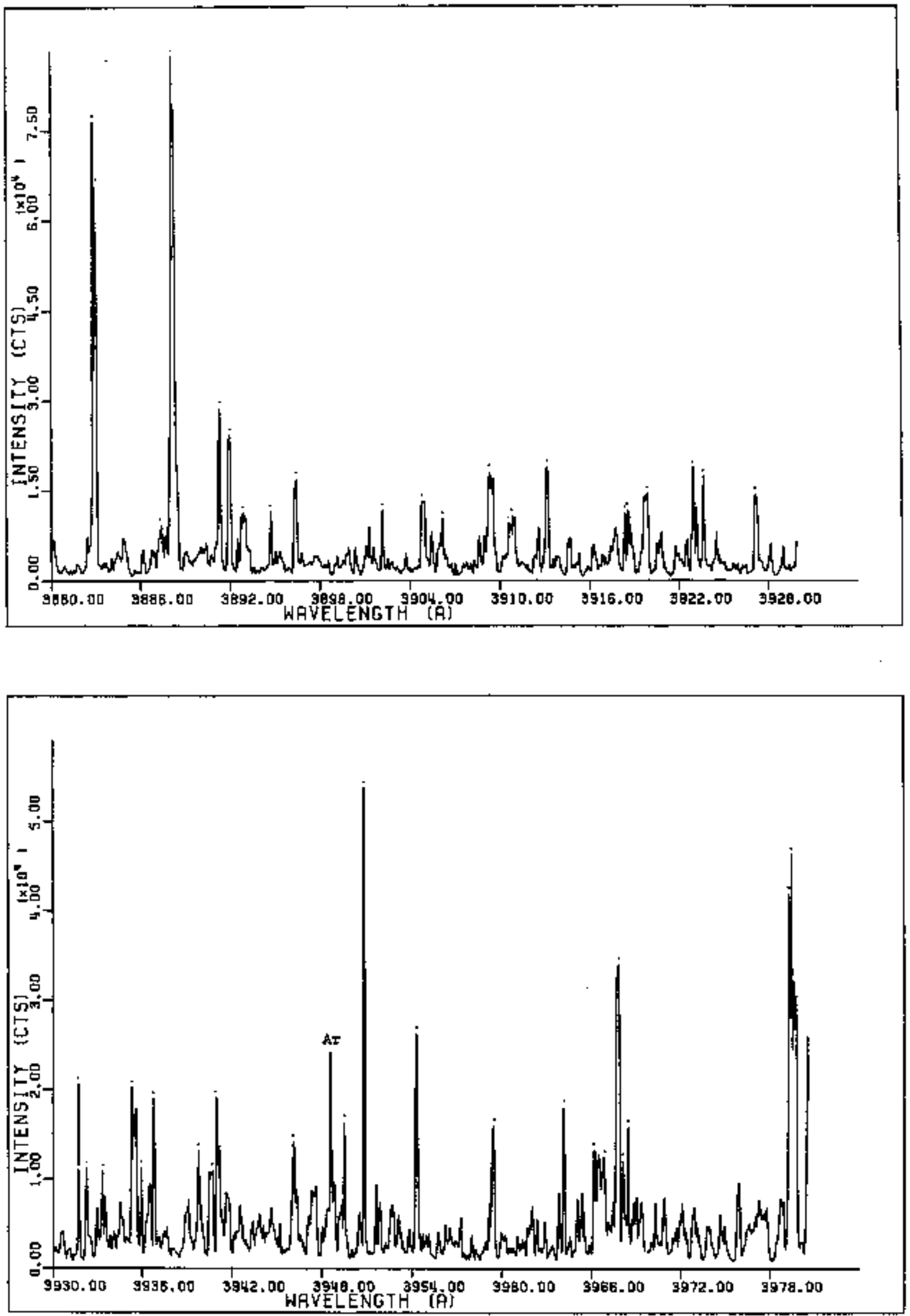


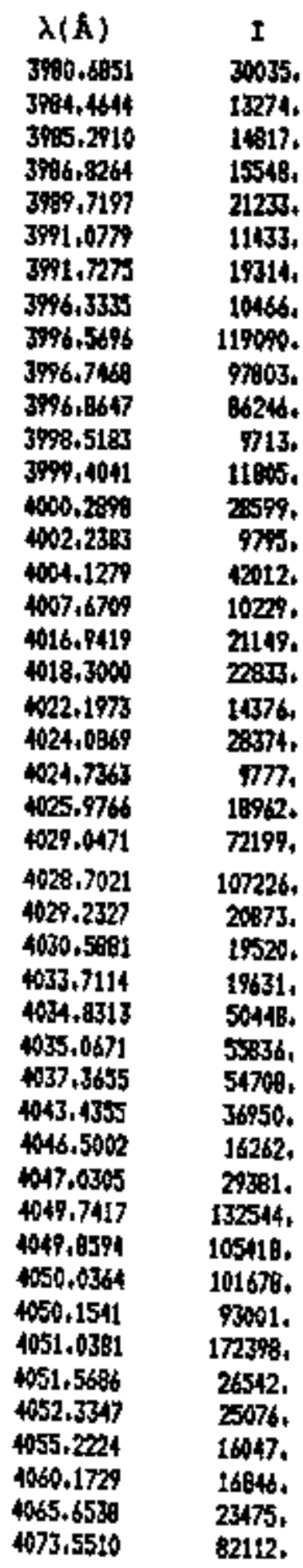



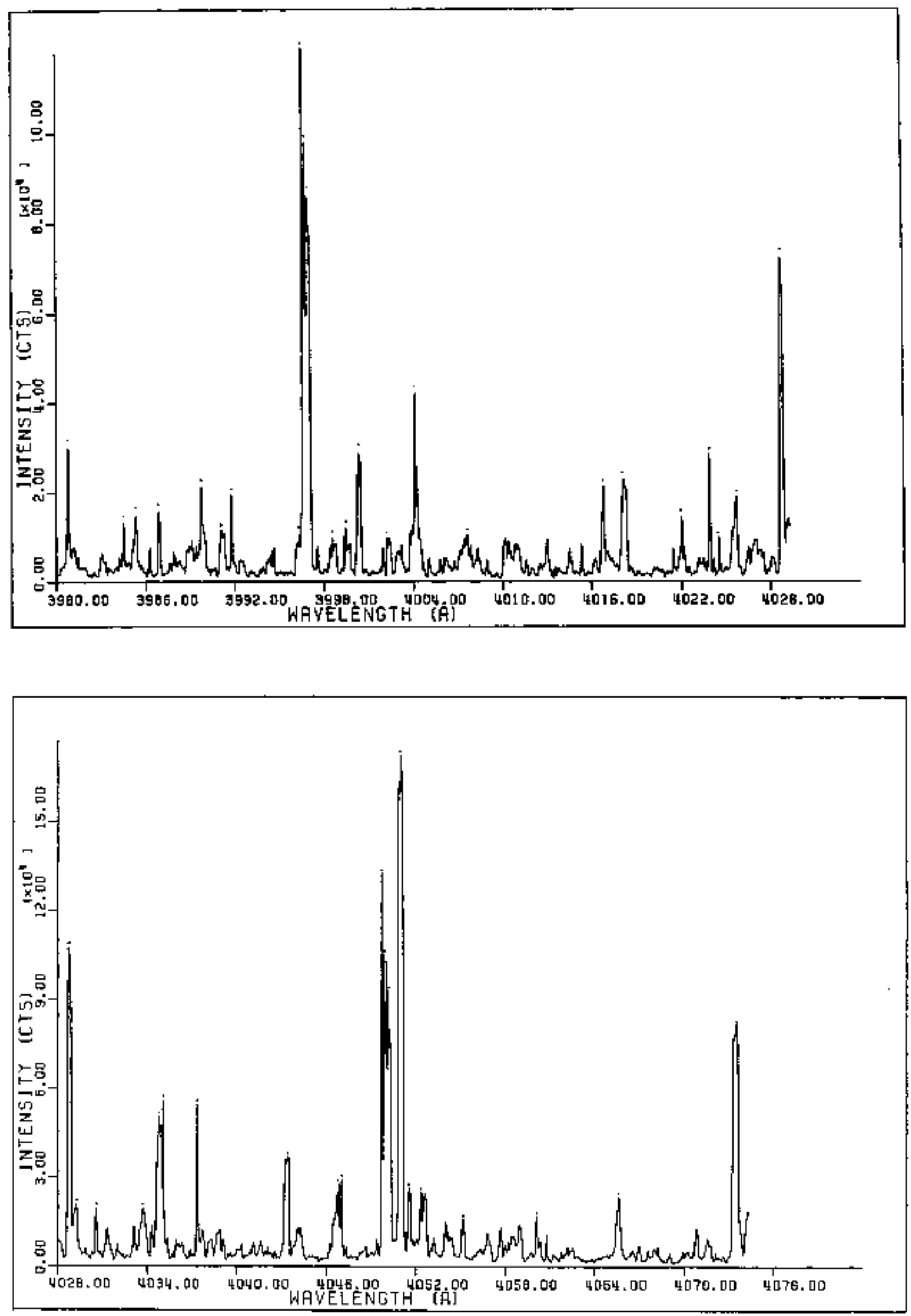


\begin{tabular}{|c|c|}
\hline$\lambda(\mathbf{A})$ & I \\
\hline 4074.0500 & 2430 \\
\hline 4075.5737 & 48027. \\
\hline $\begin{array}{l}4077,2732 \\
4079,3028\end{array}$ & $\begin{array}{l}16732, \\
14792,\end{array}$ \\
\hline 490.1415 & 17830. \\
\hline 4081,4724 & 17793, \\
\hline 4001,9026 & 1905 \\
\hline 4065.2427 & 24:123, \\
\hline 4087.0593 & 23136. \\
\hline 492,8608 & 30537. \\
\hline 4095.6736 & 30652 , \\
\hline 4098.7207 & 141941, \\
\hline 4100.2441 & 20761 \\
\hline $410 \mathrm{I}$ ،1821 & 28721 \\
\hline 4102,0610 & 19900 \\
\hline 4103.4673 & 14876 \\
\hline 4103.6431 & 115793. \\
\hline $4103.810 B$ & 15306. \\
\hline 4104.1704 & 29056 \\
\hline 4105,0498 & $\begin{array}{l}21831 \\
181039\end{array}$ \\
\hline $\begin{array}{l}4106+3996 \\
4109.7964\end{array}$ & $\begin{array}{l}181038 . \\
14605 .\end{array}$ \\
\hline 1121,0356 & 47154. \\
\hline 4123.9673 & 21164 \\
\hline $4125+3169$ & 15210 . \\
\hline $128.723 t$ & 19914 \\
\hline 4132,0747 & 55679 \\
\hline 4133,3696 & 21630. \\
\hline $4136+8452$ & 36630. \\
\hline 4137.4937 & 16513, \\
\hline $\begin{array}{l}1139.2011 \\
4143.6919\end{array}$ & $\begin{array}{l}\$ 100 . \\
26096 .\end{array}$ \\
\hline 4145.9385 & 38576 \\
\hline 4147.1807 & 24694, \\
\hline 450.3779 & 18030 \\
\hline $\begin{array}{l}4152,4521 \\
4156,7246\end{array}$ & $\begin{array}{l}21784 . \\
406 \%\end{array}$ \\
\hline $4157,310 \mathrm{~A}$ & 17194. \\
\hline 4159,1602 & 33320 \\
\hline 4160.1113 & 28173 \\
\hline 4160,4683 & $\mathbf{5 3 7 2 8}$ \\
\hline 4160.3864 & 51162 \\
\hline & 5014 \\
\hline
\end{tabular}



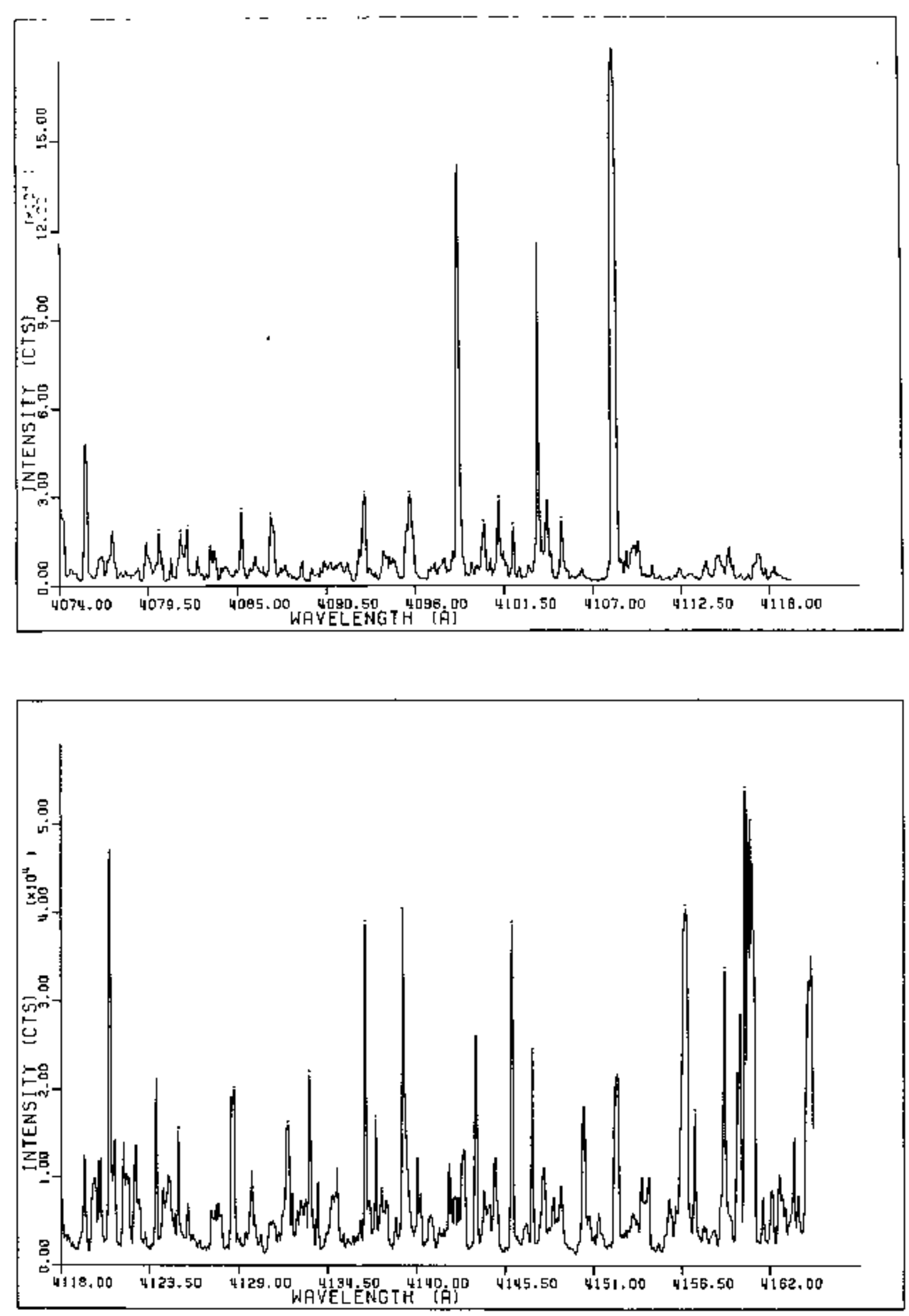


\begin{tabular}{|c|c|}
\hline$\lambda(A)$ & I \\
\hline $\begin{array}{l}1164,3950 \\
4164,8000\end{array}$ & $\begin{array}{r}394503 . \\
31 \pm 73\end{array}$ \\
\hline 4165.2769 & 101675, \\
\hline $\begin{array}{l}4166,4658 \\
4168,0674\end{array}$ & $\begin{array}{l}19544 . \\
37081 .\end{array}$ \\
\hline 4169,6006 & $\begin{array}{l}3 \% 01 . \\
23252 .\end{array}$ \\
\hline $\begin{array}{l}4171,9097 \\
4172,0273\end{array}$ & $\begin{array}{l}114203, \\
101164 .\end{array}$ \\
\hline $\begin{array}{l}4172,[453 \\
4172,2632\end{array}$ & $\begin{array}{l}80500 . \\
8576 \% .\end{array}$ \\
\hline $\begin{array}{l}4172.5576 \\
4180.6401\end{array}$ & $\begin{array}{l}20+29 . \\
30026 .\end{array}$ \\
\hline $\begin{array}{l}1182.1523 \\
4186.037\end{array}$ & \\
\hline 9192,8774 & 64830 . \\
\hline $4197,15 \mathrm{AB}$ & 37060. \\
\hline $\begin{array}{l}4199,7060 \\
4200,7032\end{array}$ & 49670. \\
\hline 4206.6938 & 6655. \\
\hline 4207.3662 & 29169. \\
\hline $4208+4795$ & IB119, \\
\hline 4209.6675 & 21865 \\
\hline 4211,7676 & 14231. \\
\hline 4212.9321 & 18491. \\
\hline 4216.3574 & t6513. \\
\hline 4217.6313 & 24611. \\
\hline 4221.3833 & 20283. \\
\hline 424.2002 & $14702+$ \\
\hline $\begin{array}{l}4227+5226 \\
4229,6047\end{array}$ & 74350 \\
\hline $\begin{array}{l}4229,6047 \\
4234.6914\end{array}$ & $\begin{array}{l}19289, \\
89976 .\end{array}$ \\
\hline 4235.9365 & 18345. \\
\hline 1236.3007 & 14372. \\
\hline 4237.2925 & 31032. \\
\hline 4237.9697 & 50900 . \\
\hline 4230,1372 & 39399 \\
\hline $4213.590 \mathrm{~A}$ & 16012. \\
\hline 4245.1572 & 15269 \\
\hline 247,811 & 31909. \\
\hline $\begin{array}{l}4250,0374 \\
4201.5029\end{array}$ & 2060: \\
\hline 4201.5029 & \\
\hline
\end{tabular}

$$
-58-
$$



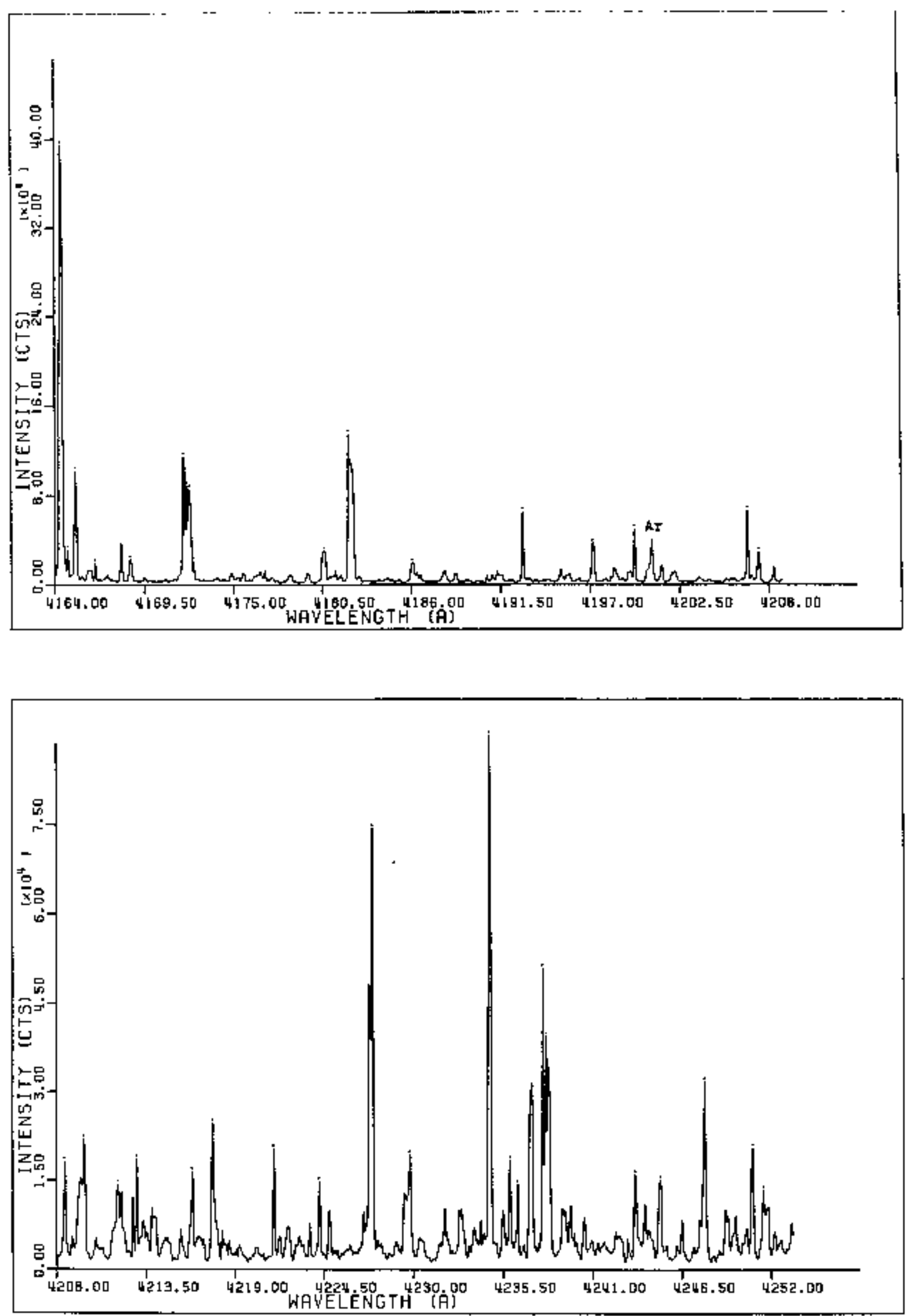


\begin{tabular}{|c|c|}
\hline$\lambda(\mathbf{A})$ & I \\
\hline 1256.6108 & 142099. \\
\hline 4258,0537 & 10571 , \\
\hline $4258+6304$ & 15729, \\
\hline $4257+3804$ & 15977 , \\
\hline 4261.5137 & 35439. \\
\hline 4265.6611 & 15715. \\
\hline 4269,0557 & 23383 \\
\hline 4269,7461 & 51312. \\
\hline 4272,1602 & 19202 \\
\hline 4273,1367 & 122961, \\
\hline 4273.8262 & 22954 \\
\hline 1274,6299 & 16717. \\
\hline 4276.1226 & 17658. \\
\hline 4277,4995 & 21250 \\
\hline 4279.4497 & 6771 \\
\hline 1282.3154 & 10000, \\
\hline 2882,5444 & 40702 . \\
\hline 4286,4386 & 52623. \\
\hline 4297.8682 & 25593. \\
\hline 4289.5935 & 105352, \\
\hline 4290.7837 & 469546. \\
\hline 4293.2402 & $1369 \%$ \\
\hline 1295,9805 & 93702. \\
\hline 4301.6870 & 13659 \\
\hline $4302+9478$ & 30252 \\
\hline 1304,6665 & 25734. \\
\hline 4307,1865 & 12670 \\
\hline $\begin{array}{l}4307,7017 \\
4307,9878\end{array}$ & $\begin{array}{r}136644 \\
12774\end{array}$ \\
\hline $\begin{array}{l}5197,4876 \\
4312.1357\end{array}$ & 2790, \\
\hline 4311.5361 & 159 15. \\
\hline 1313.1377 & 17228, \\
\hline 4314,2817 & 23403 \\
\hline 4316,4541 & 31381. \\
\hline 4318.1113 & 4089 \\
\hline 4319,6543 & 5994 \\
\hline 1321.1963 & 26905. \\
\hline 4222.7333 & $2310 \%$ \\
\hline 4325.6774 & 13650. \\
\hline 4327.5322 & 13878 \\
\hline 4332.0937 & $\$ 176 \%$ \\
\hline $4333+5 \% 7$ & 3462, \\
\hline 4336.4810 & [34701 \\
\hline
\end{tabular}



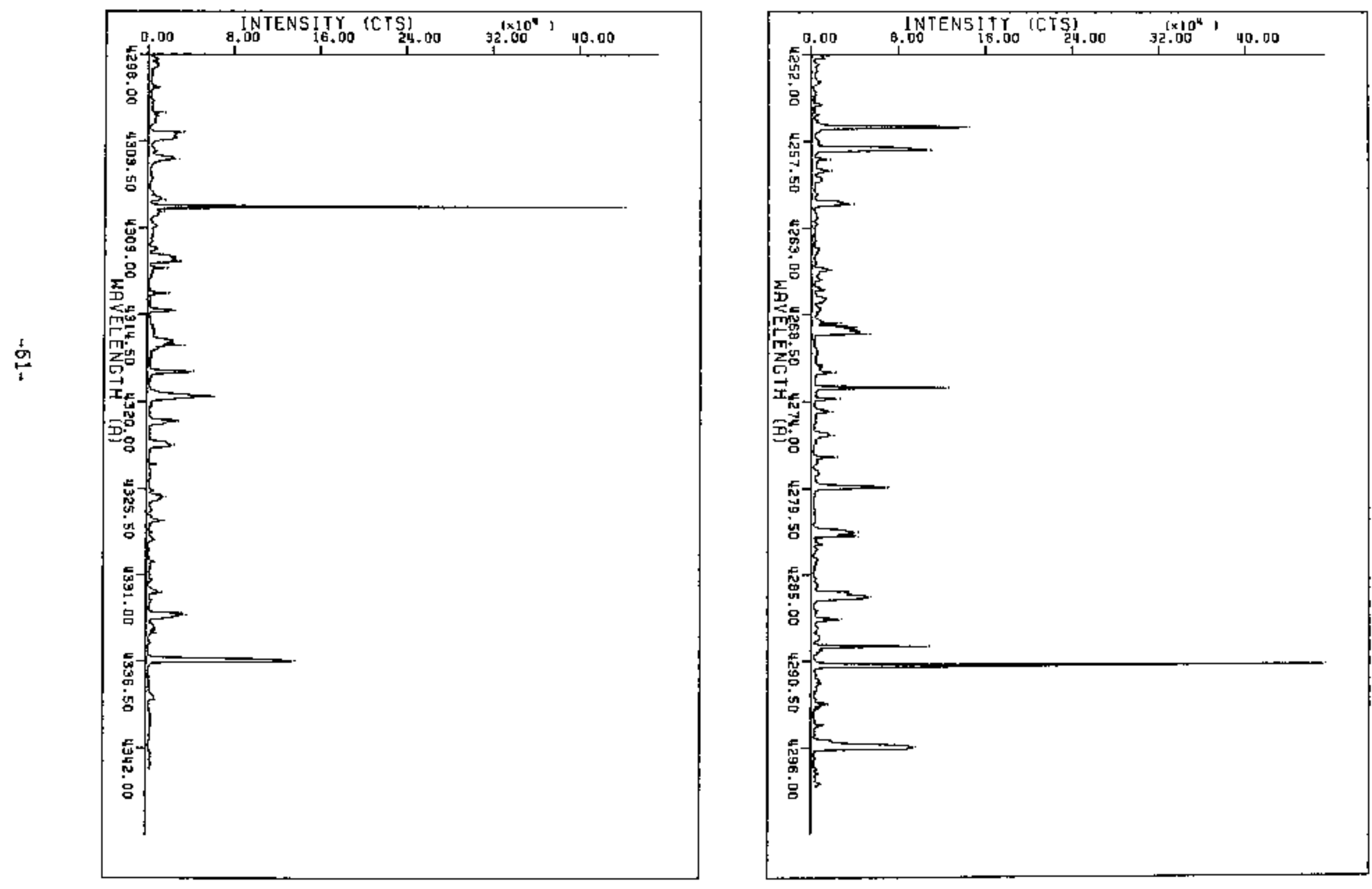


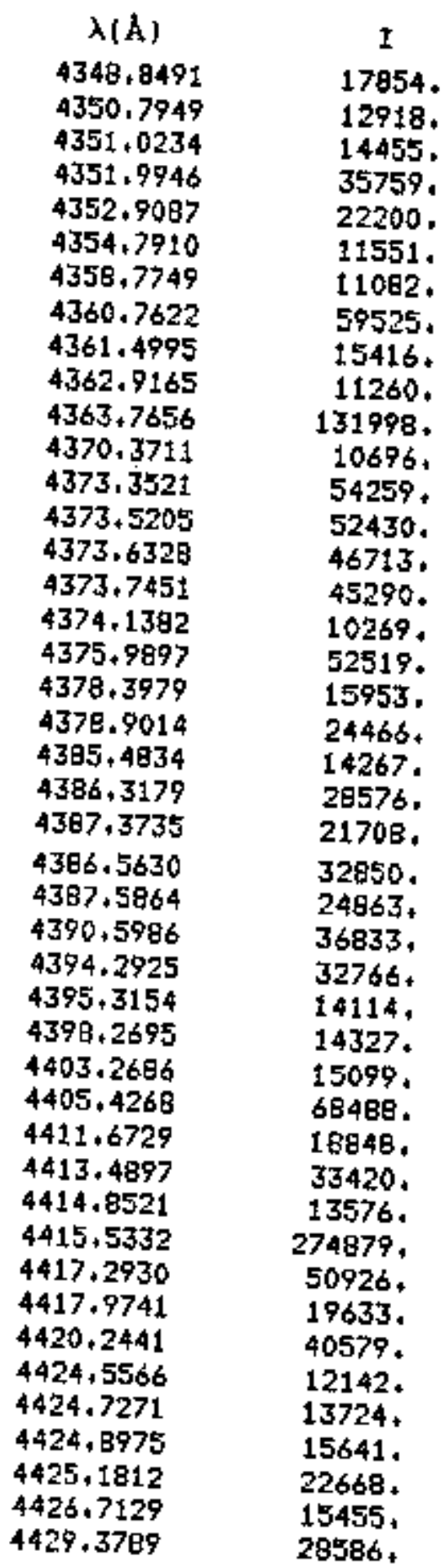



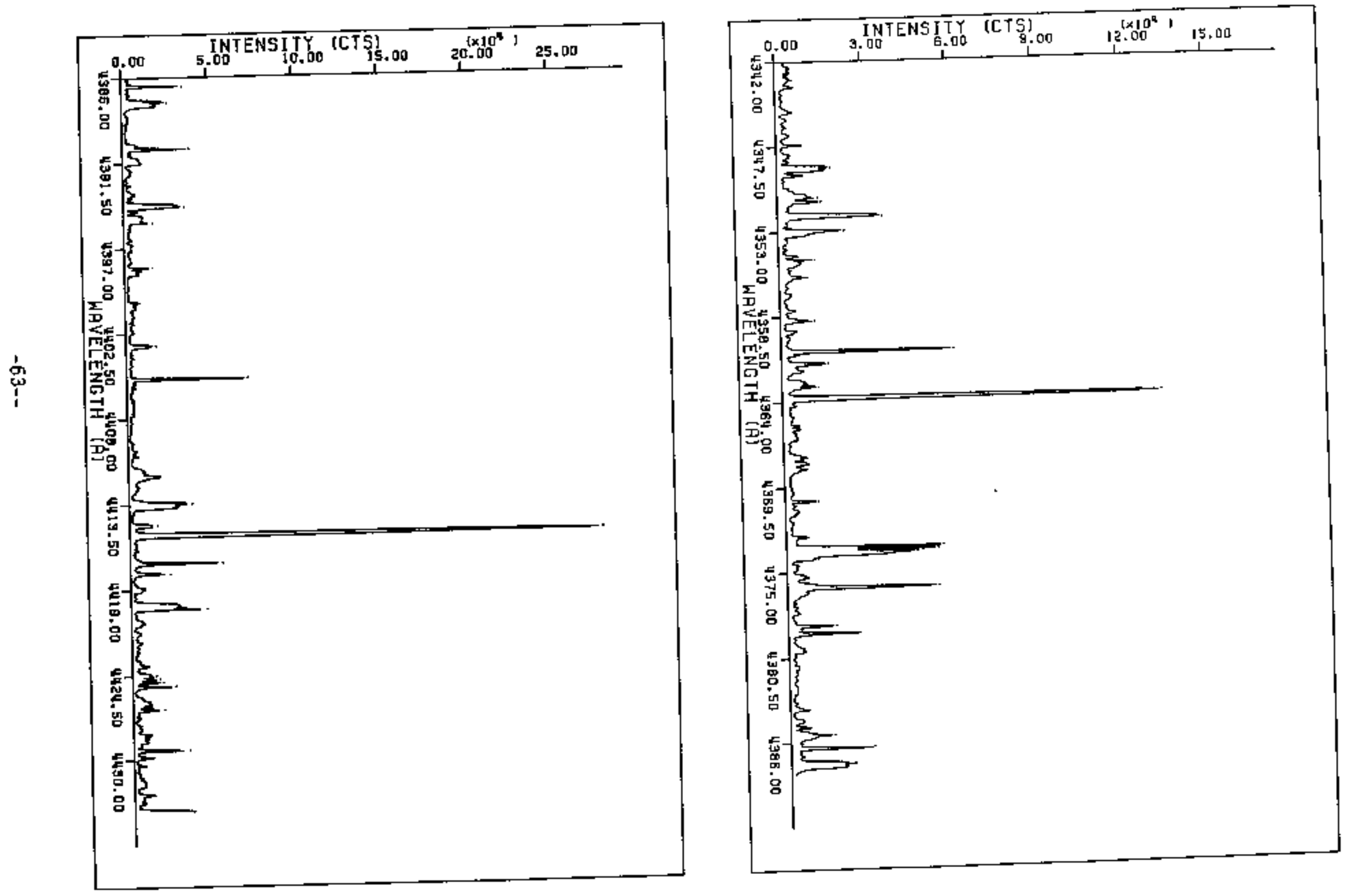


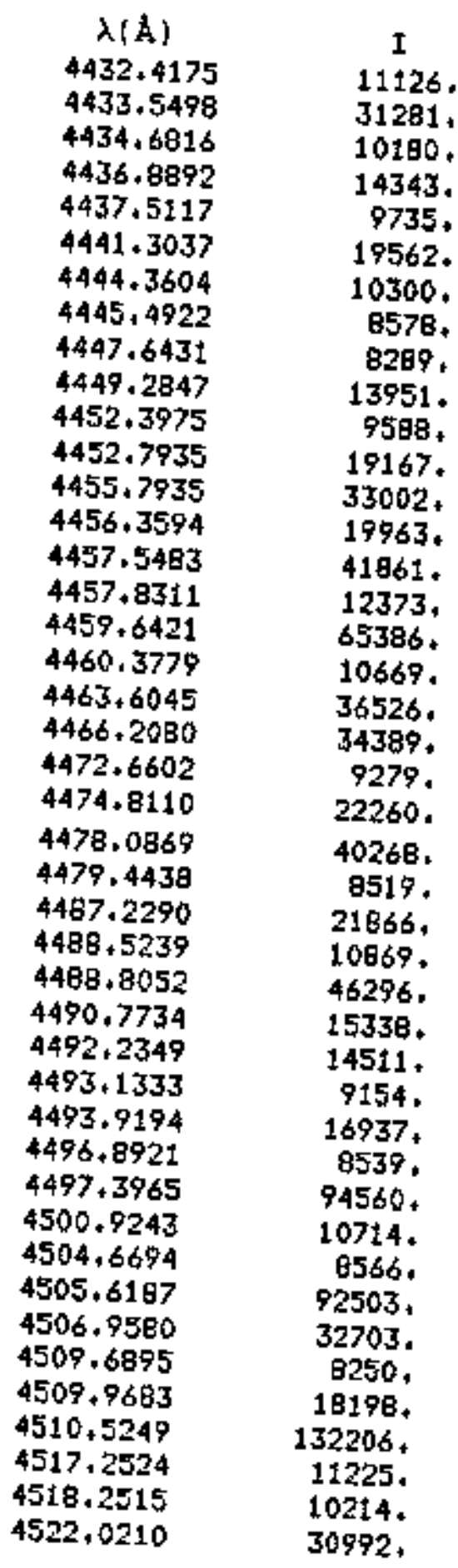



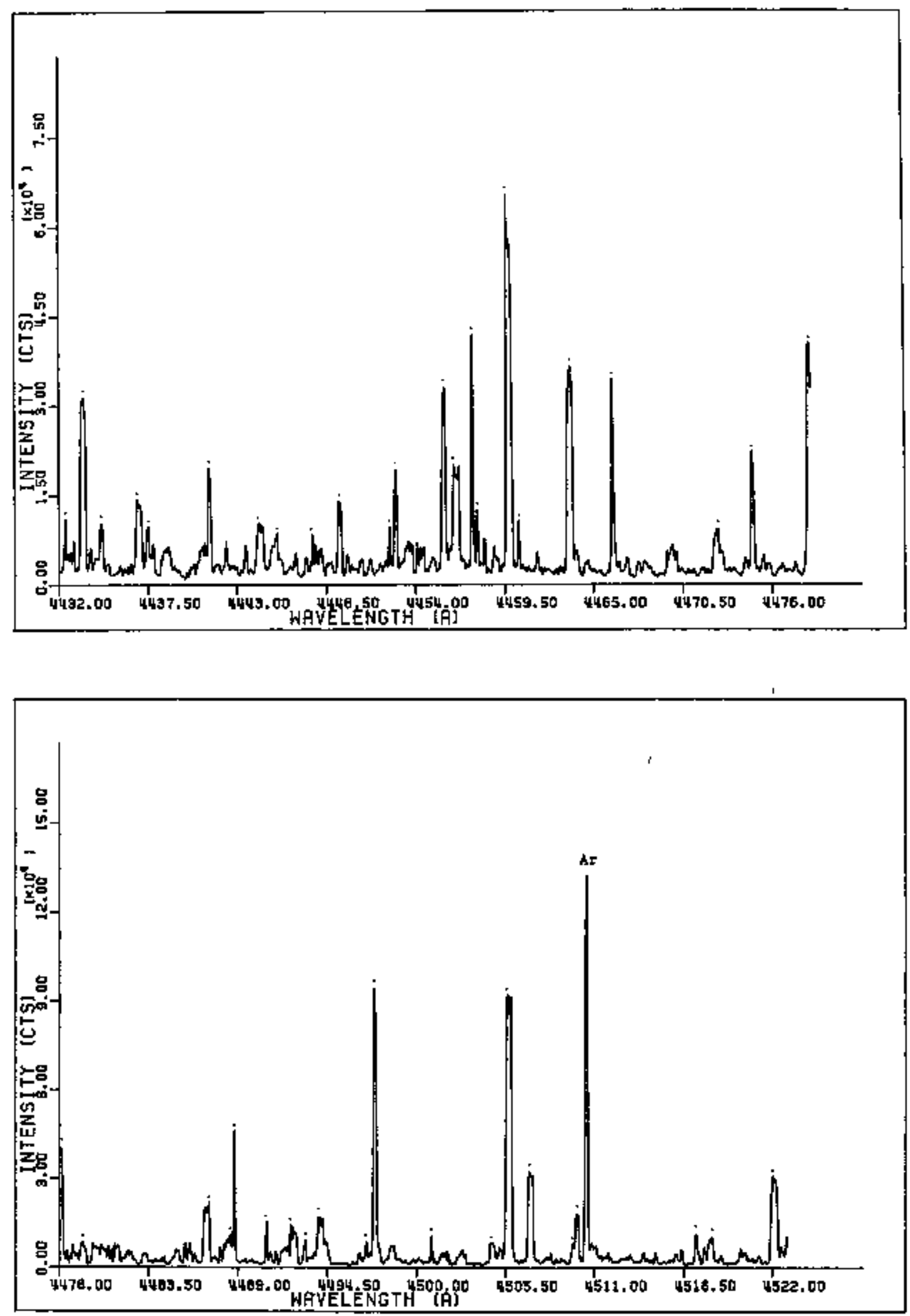


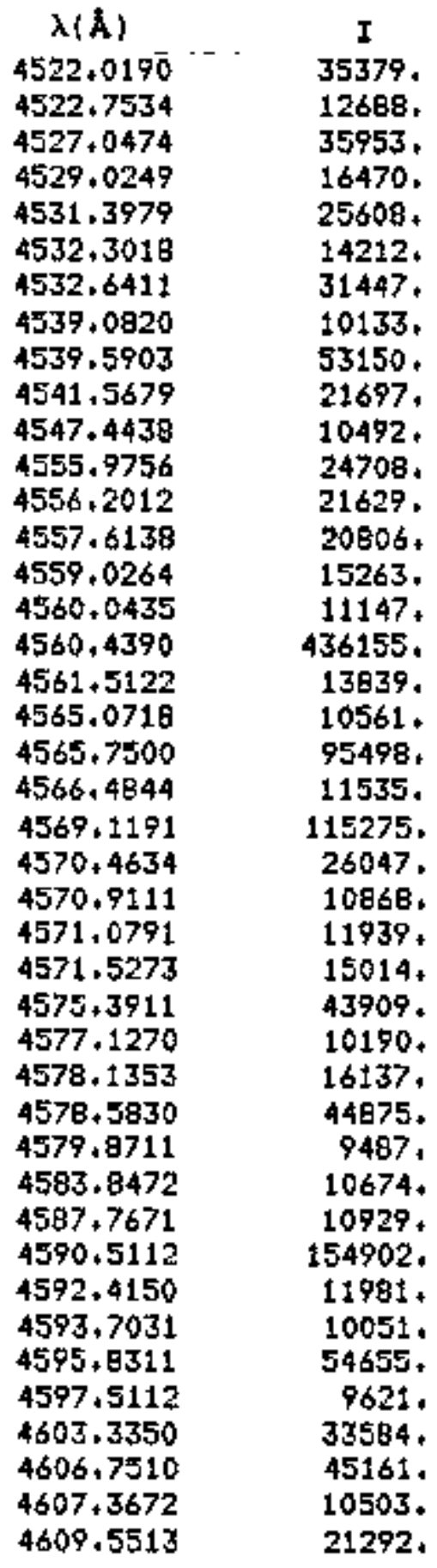



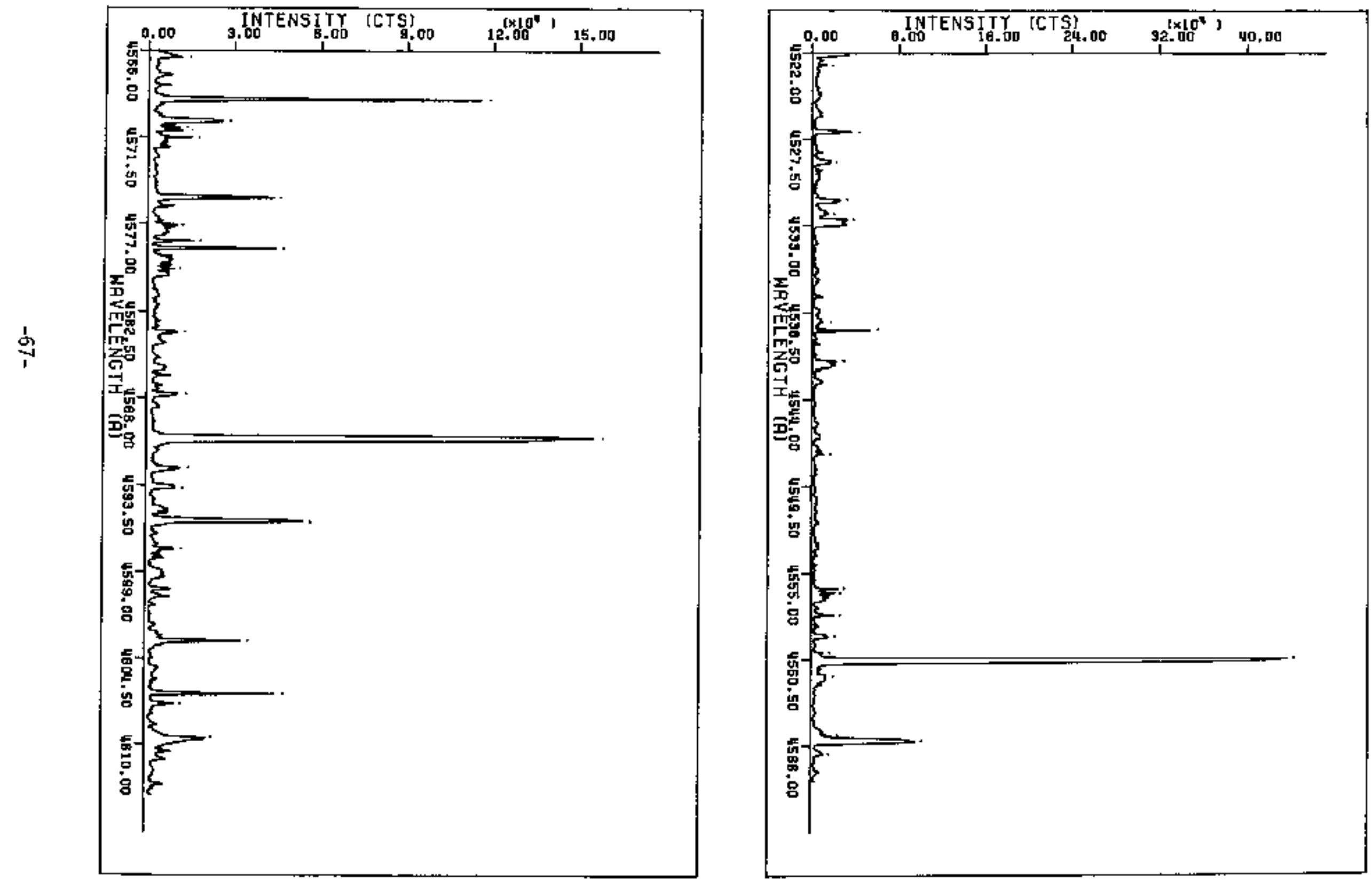


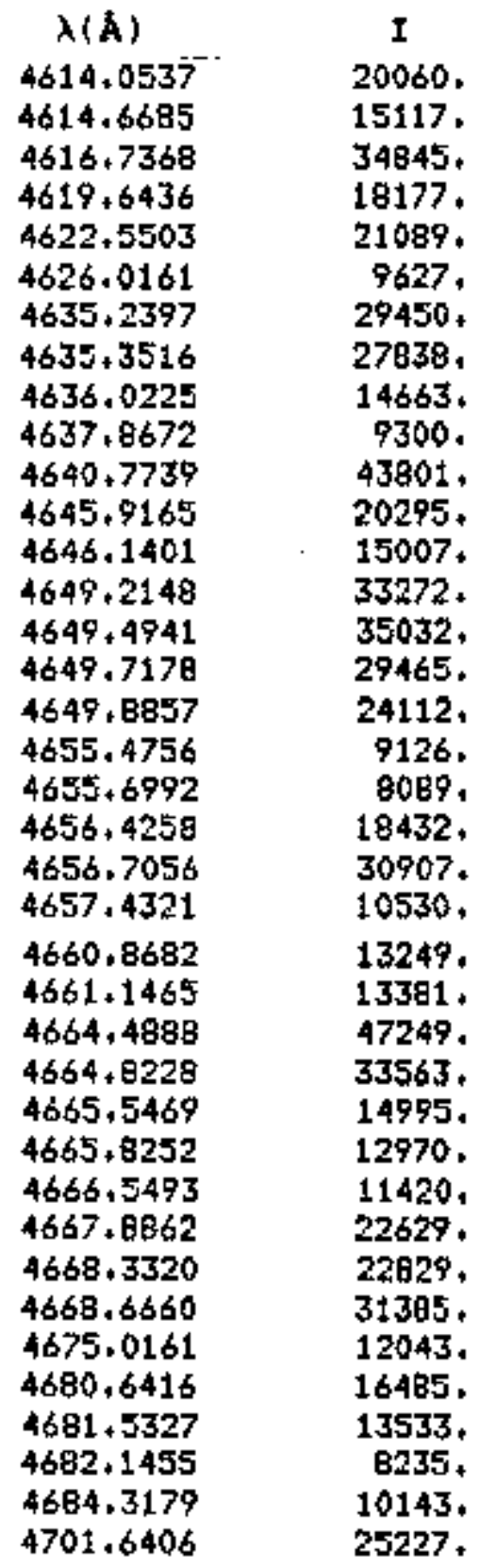



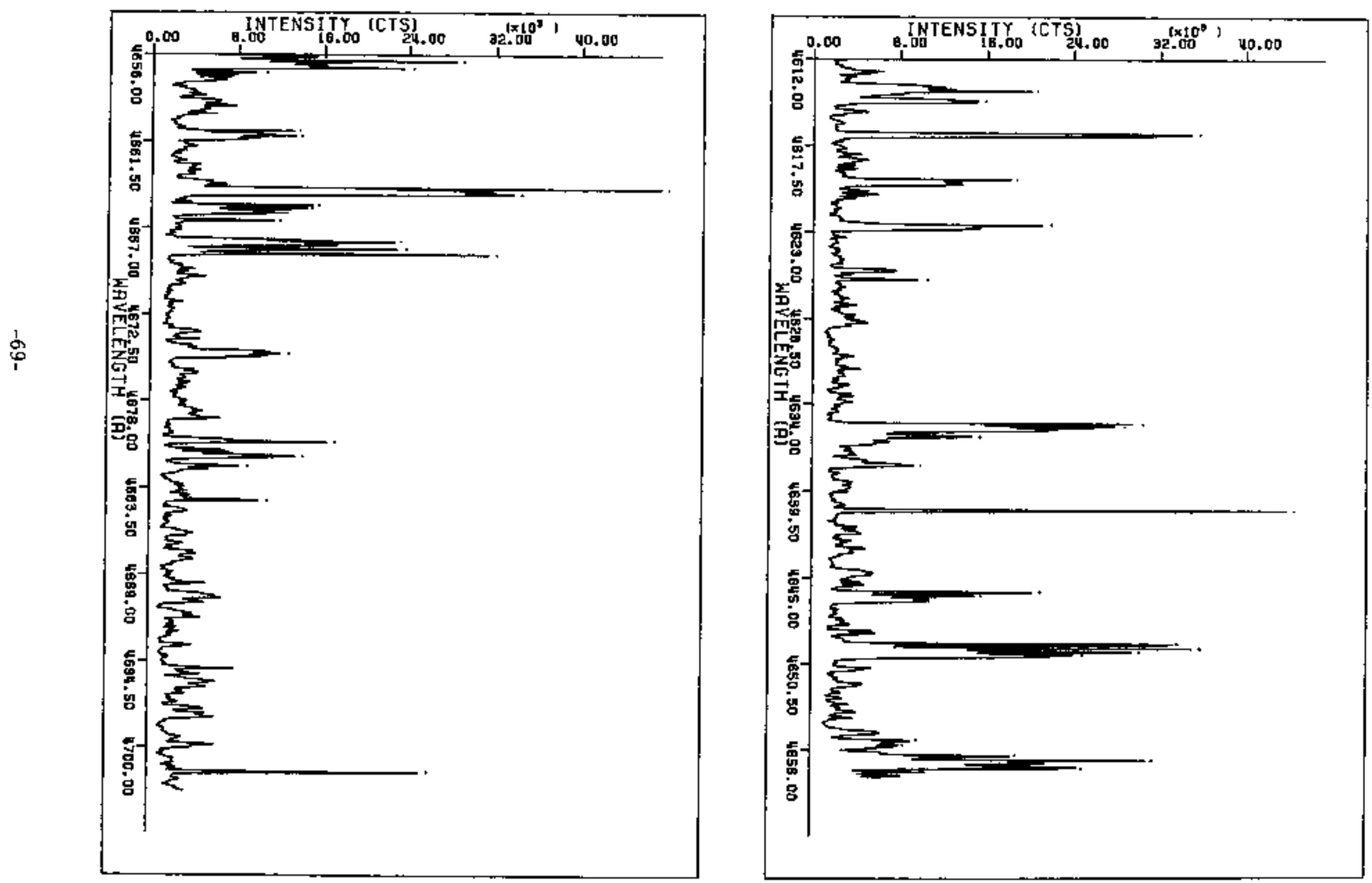


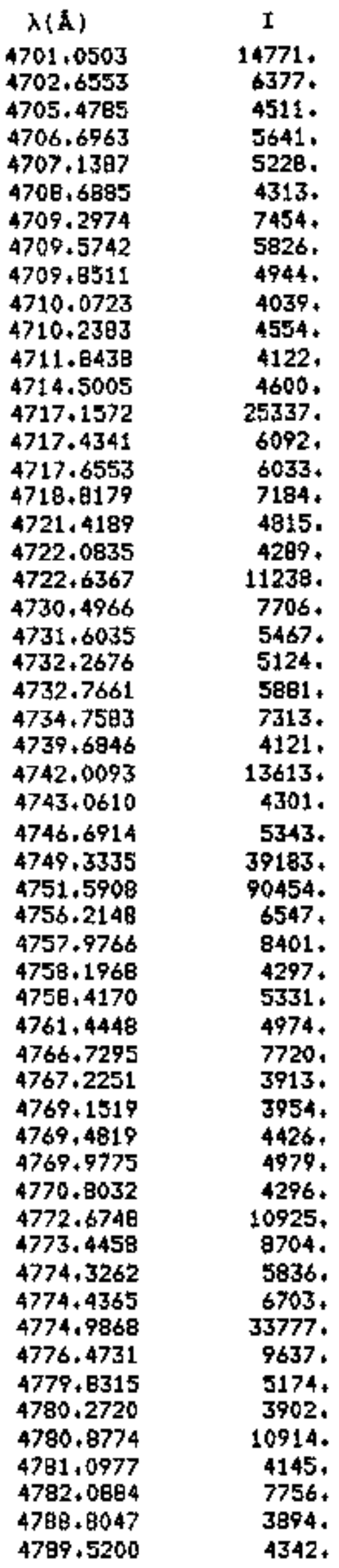



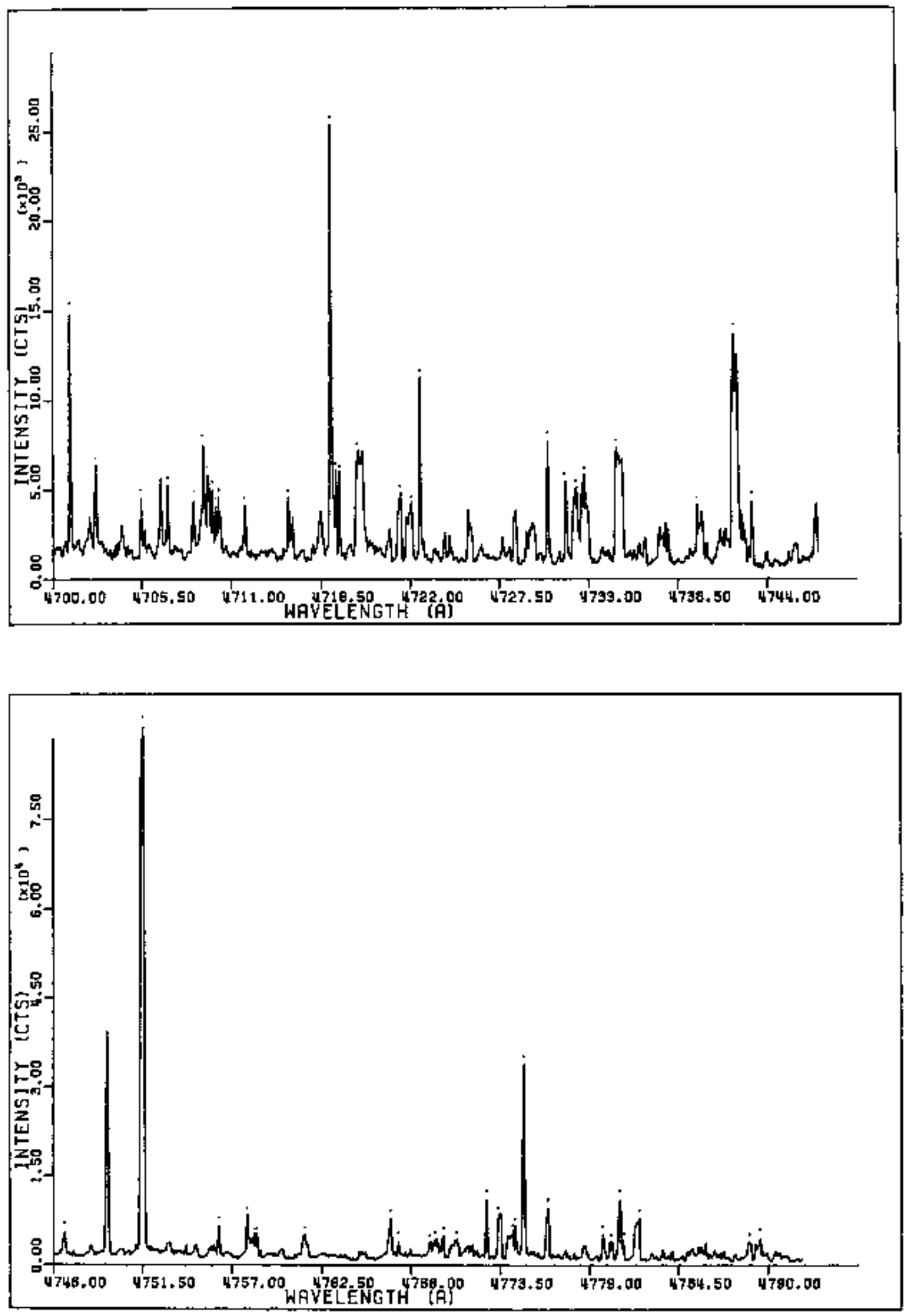


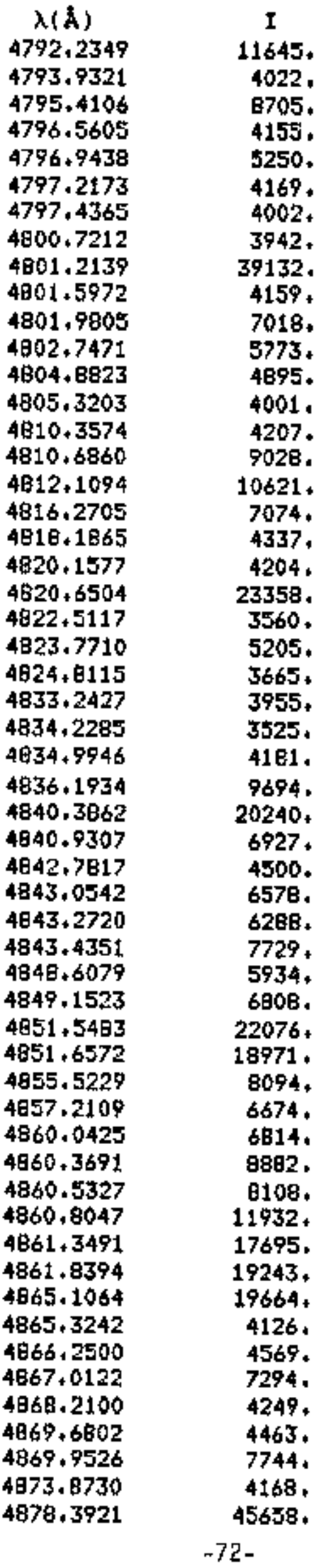



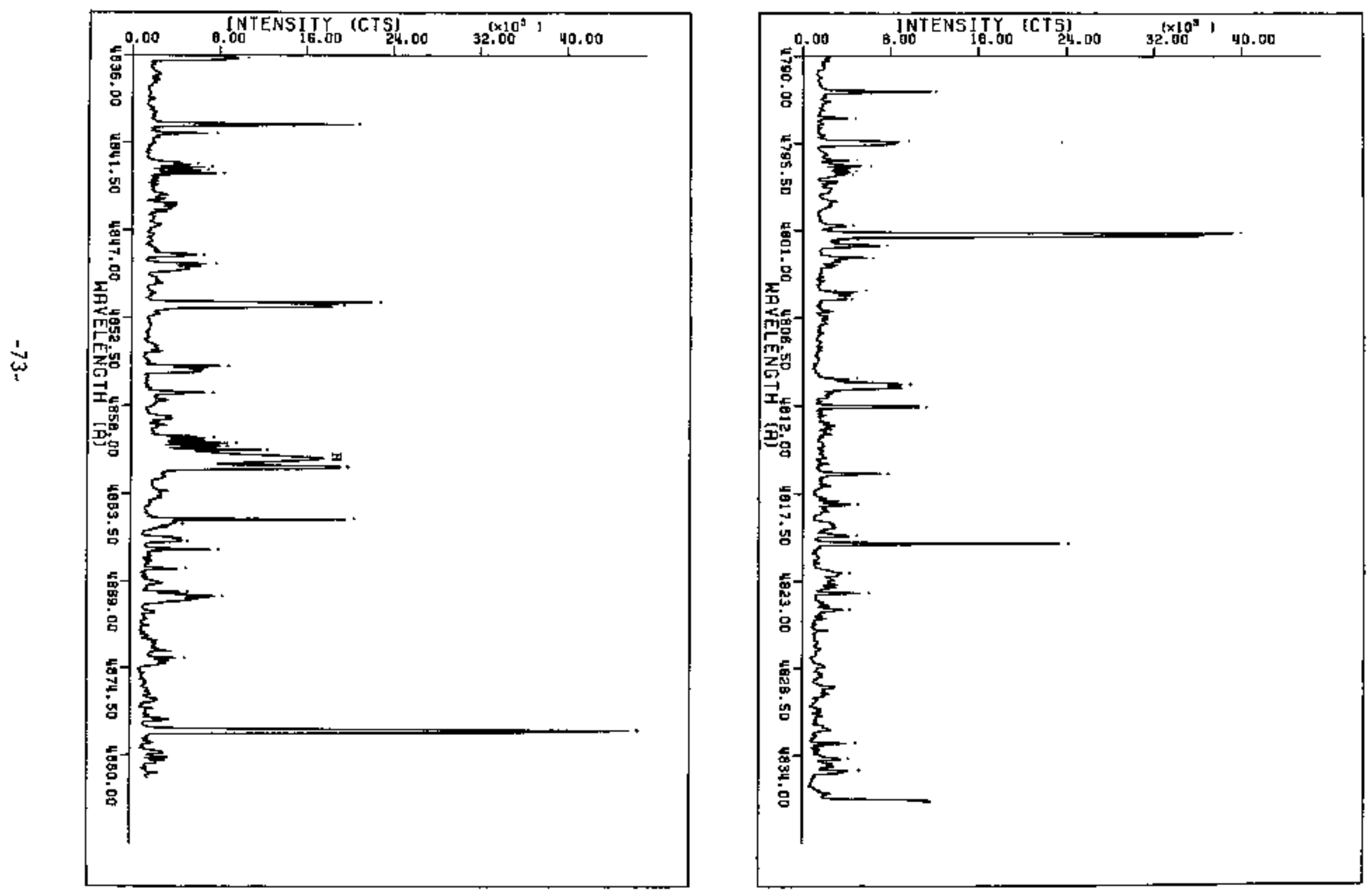


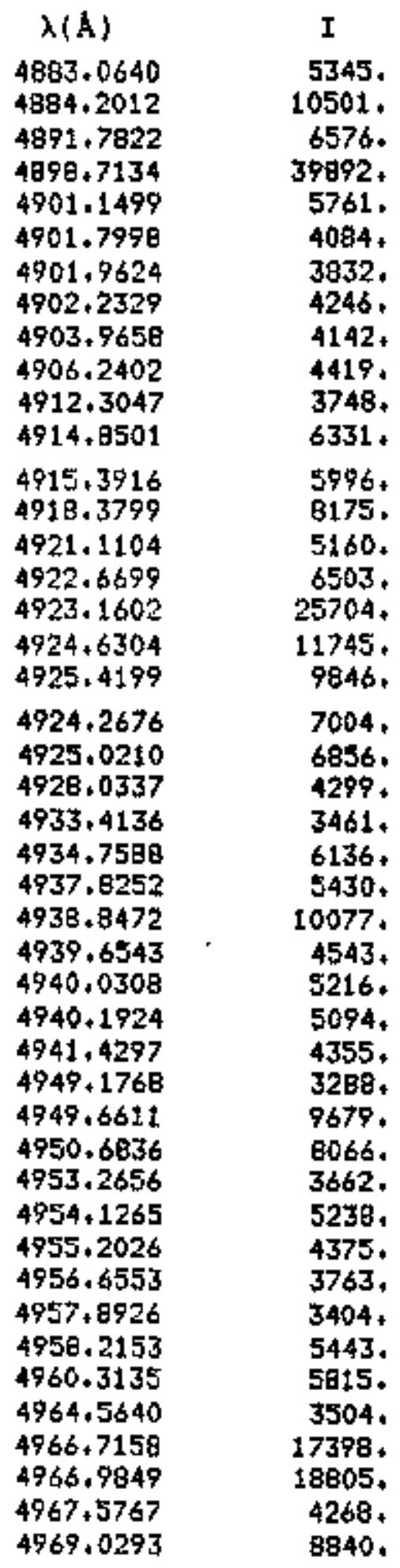



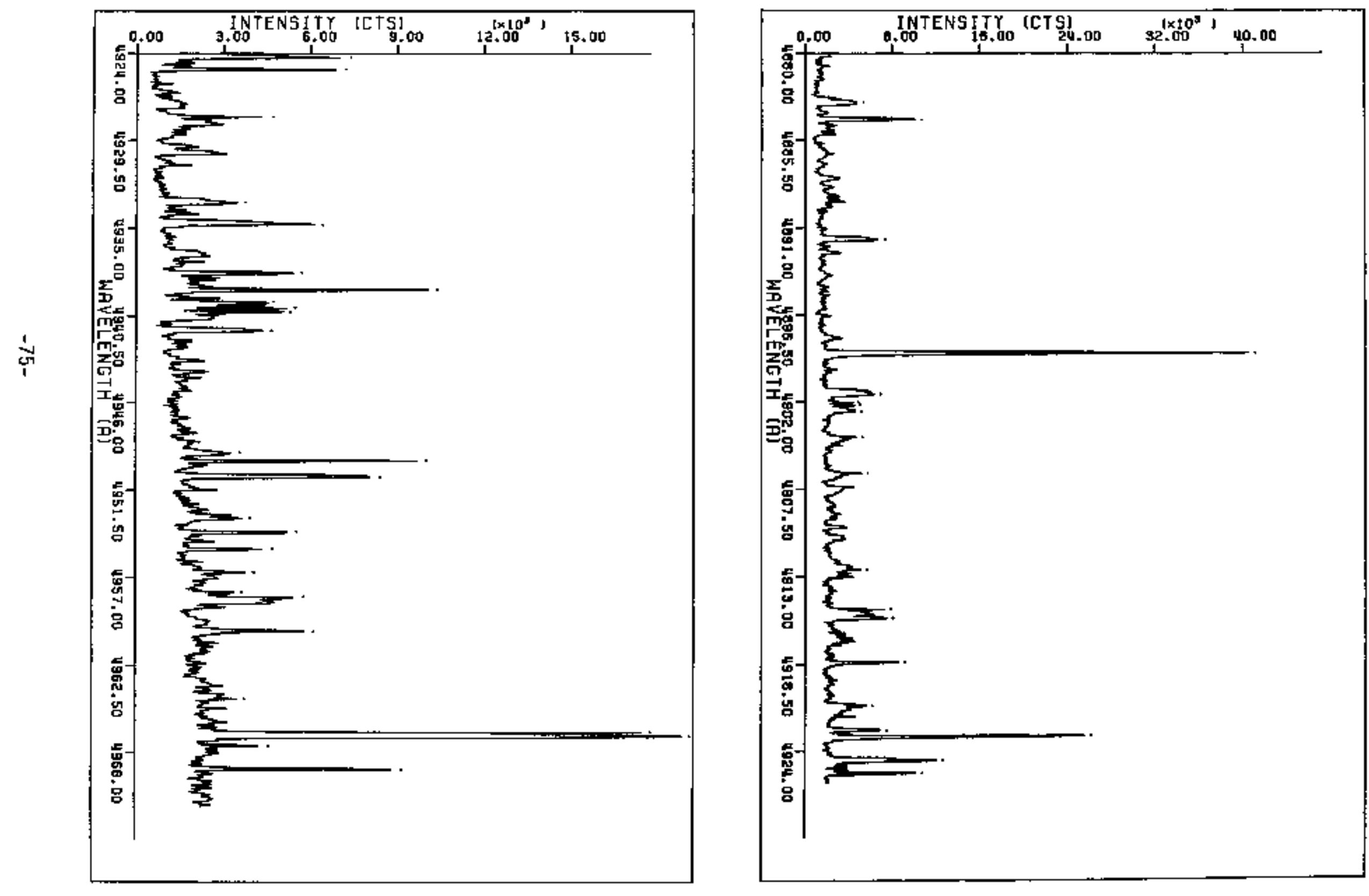


\begin{tabular}{|c|c|}
\hline$\lambda(A)$ & I \\
\hline $\begin{array}{l}4977.9292 \\
4978+0898 \\
4978.6245 \\
4991.0854\end{array}$ & $\begin{array}{l}2971 \text {. } \\
3009 . \\
4896 . \\
4369 .\end{array}$ \\
\hline $\begin{array}{l}4981.6206 \\
4986.0078 \\
4968.7363\end{array}$ & $\begin{array}{r}12779, \\
12554, \\
3761 .\end{array}$ \\
\hline $\begin{array}{l}4991.6797 \\
4992.4277 \\
4992.9629\end{array}$ & $\begin{array}{l}3116 . \\
4717 . \\
5887 .\end{array}$ \\
\hline $\begin{array}{l}4993.6582 \\
4995+7993 \\
4997+9380\end{array}$ & $\begin{array}{l}2932 . \\
3579 \text {. } \\
3591 .\end{array}$ \\
\hline $\begin{array}{l}4998.0996 \\
4999+00 \mathrm{B3} \\
5000.5596\end{array}$ & $\begin{array}{r}3491 . \\
3068, \\
11618 .\end{array}$ \\
\hline $\begin{array}{l}5002+9136 \\
5003+6626 \\
5004+7861\end{array}$ & $\begin{array}{l}4204 . \\
7752 . \\
3259 .\end{array}$ \\
\hline $\begin{array}{l}5005+1069 \\
5006+9795 \\
5010.0293\end{array}$ & $\begin{array}{l}2842, \\
5464, \\
5024 .\end{array}$ \\
\hline $\begin{array}{l}5012.0080 \\
5013.7744 \\
5013.9883\end{array}$ & $\begin{array}{l}2895 . \\
6247 . \\
2949 .\end{array}$ \\
\hline $\begin{array}{l}5014.5767 \\
5014.9512\end{array}$ & $\begin{array}{l}3661, \\
3477 .\end{array}$ \\
\hline $\begin{array}{l}5015+8174 \\
5024,2764 \\
5024.3828 \\
5024,4888 \\
5024.6484\end{array}$ & $\begin{array}{r}11407 . \\
7695 . \\
9625 . \\
8135 . \\
10911 .\end{array}$ \\
\hline $\begin{array}{l}5024.8081 \\
5026.0850 \\
5026.2979\end{array}$ & $\begin{array}{l}4325 \\
3457 \\
3479\end{array}$ \\
\hline $\begin{array}{l}5026+5107 \\
5030.6602 \\
5031.5112\end{array}$ & $\begin{array}{r}4912 . \\
5051 . \\
10533\end{array}$ \\
\hline $\begin{array}{l}5032+0435 \\
5036+4590 \\
5039,1191\end{array}$ & $\begin{array}{r}13625 . \\
3479 \\
3438 .\end{array}$ \\
\hline $\begin{array}{l}5039.7573 \\
5040.0767 \\
5040.2896\end{array}$ & $\begin{array}{l}3904+ \\
4125 . \\
4136 .\end{array}$ \\
\hline $\begin{array}{l}5040.8745 \\
5041.7780 \\
5043.8008\end{array}$ & $\begin{array}{l}3791 . \\
3424 . \\
8065 .\end{array}$ \\
\hline $\begin{array}{l}5043.9072 \\
5044.0664 \\
5044+2793\end{array}$ & $\begin{array}{l}9925 . \\
5271 . \\
7172+\end{array}$ \\
\hline $\begin{array}{l}5046+8330 \\
5048,6948 \\
5054.4937\end{array}$ & $\begin{array}{l}3447 . \\
4560 \text {. } \\
6142 .\end{array}$ \\
\hline $\begin{array}{l}5054.6001 \\
5055.5044\end{array}$ & $\begin{array}{l}8174, \\
4224 .\end{array}$ \\
\hline
\end{tabular}



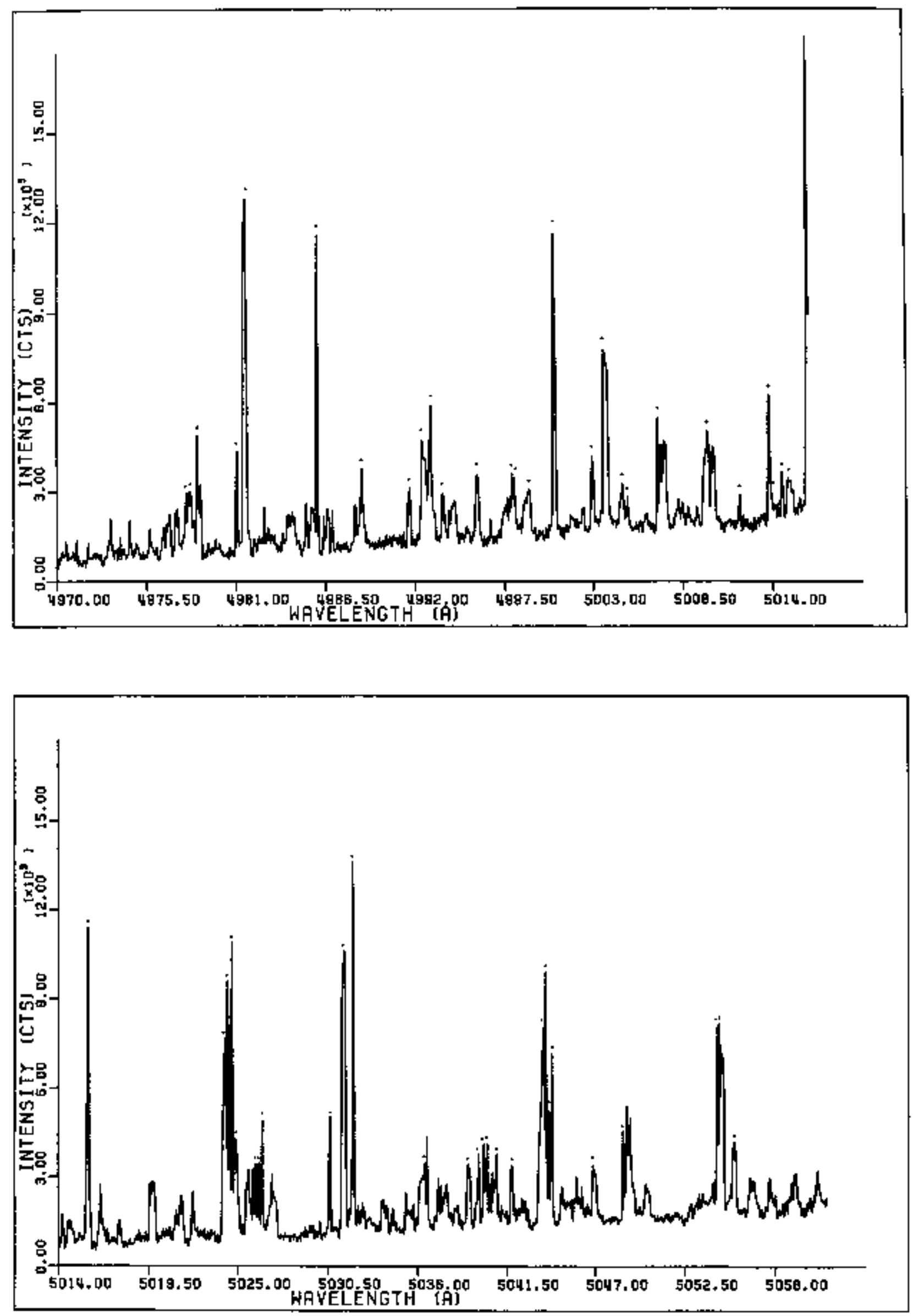


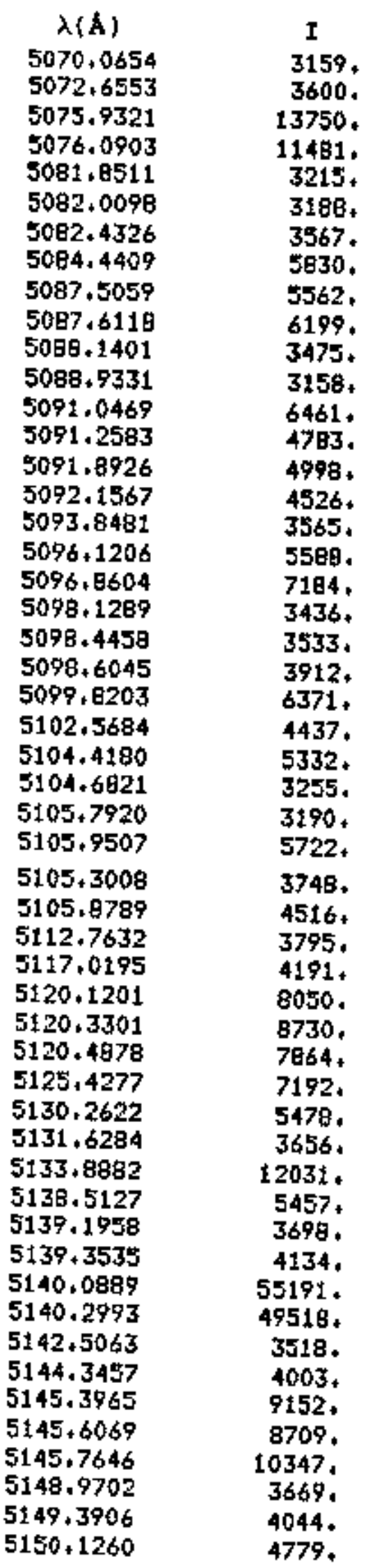



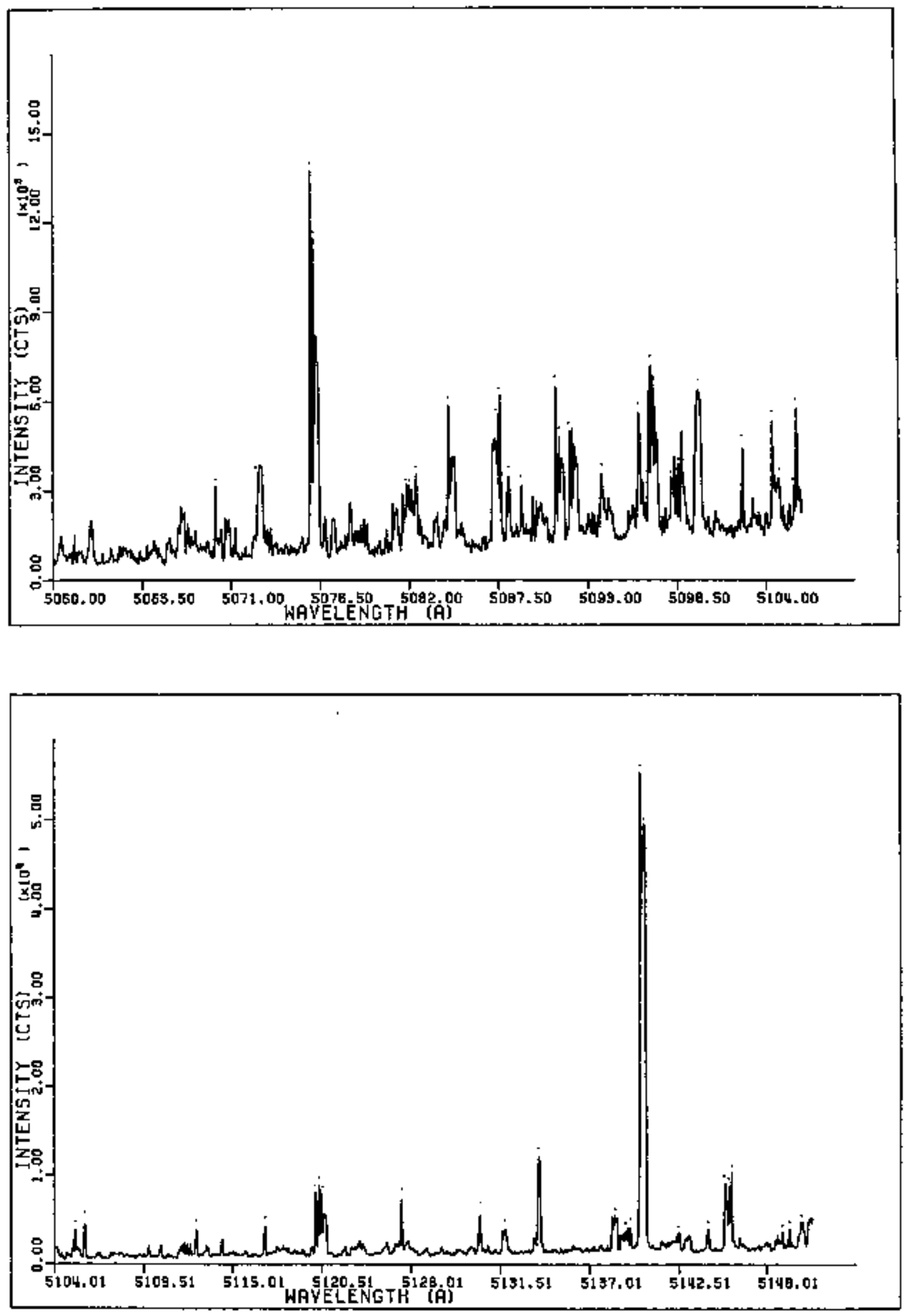


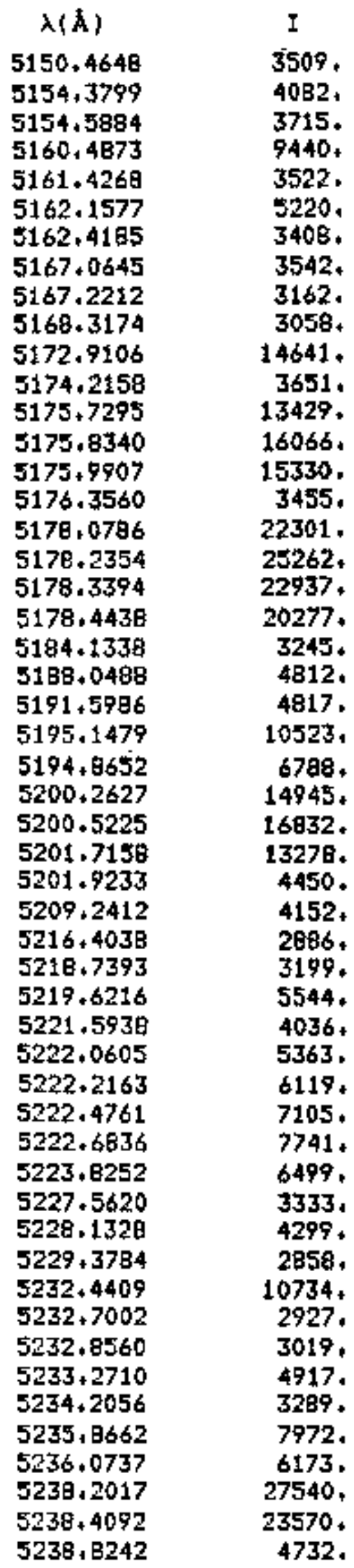



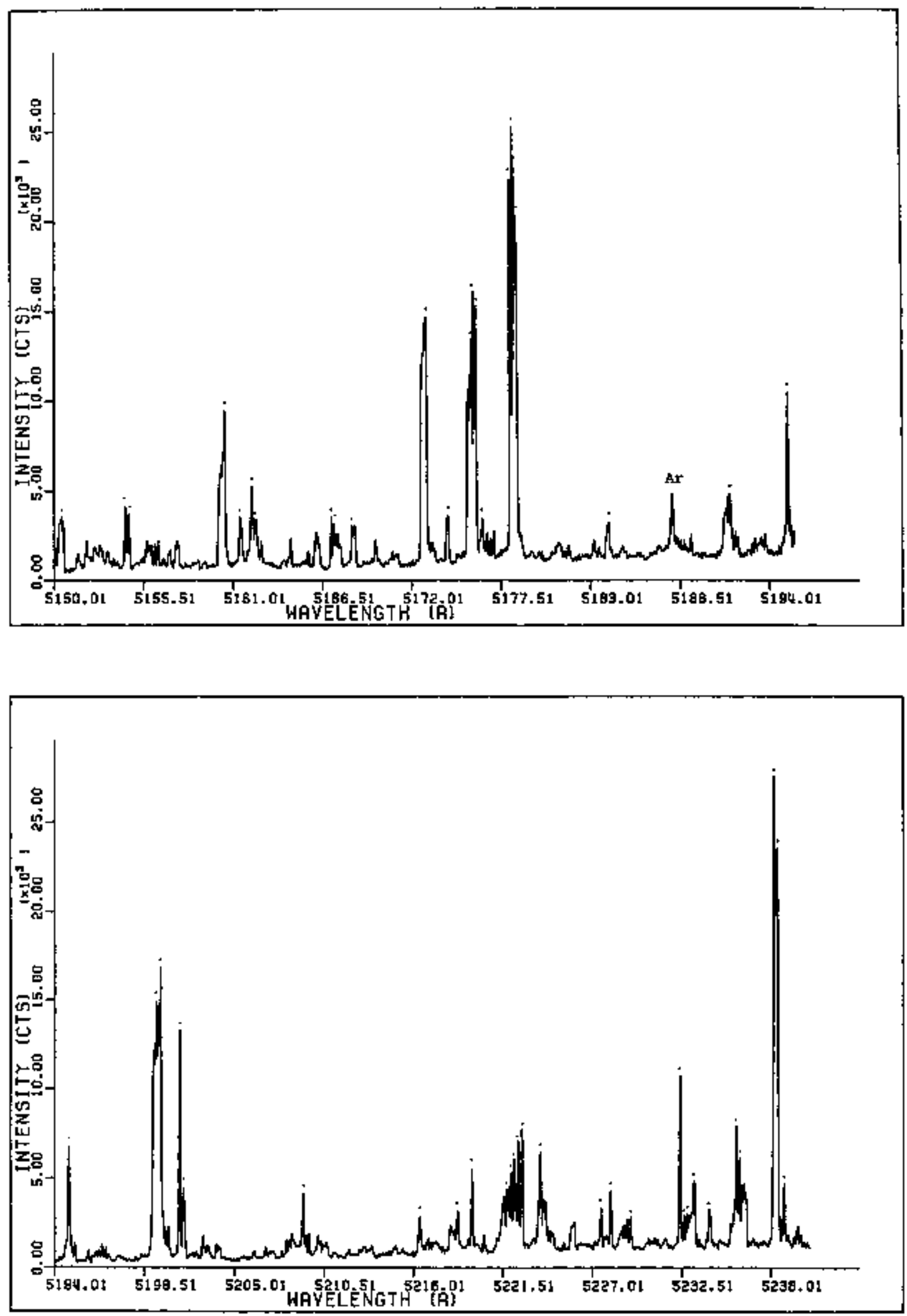


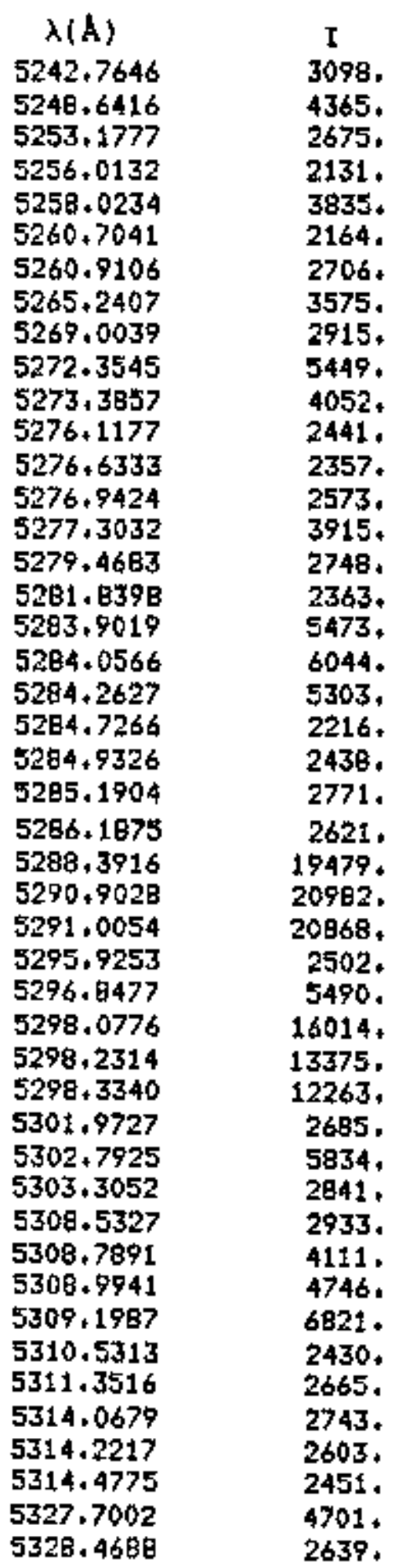



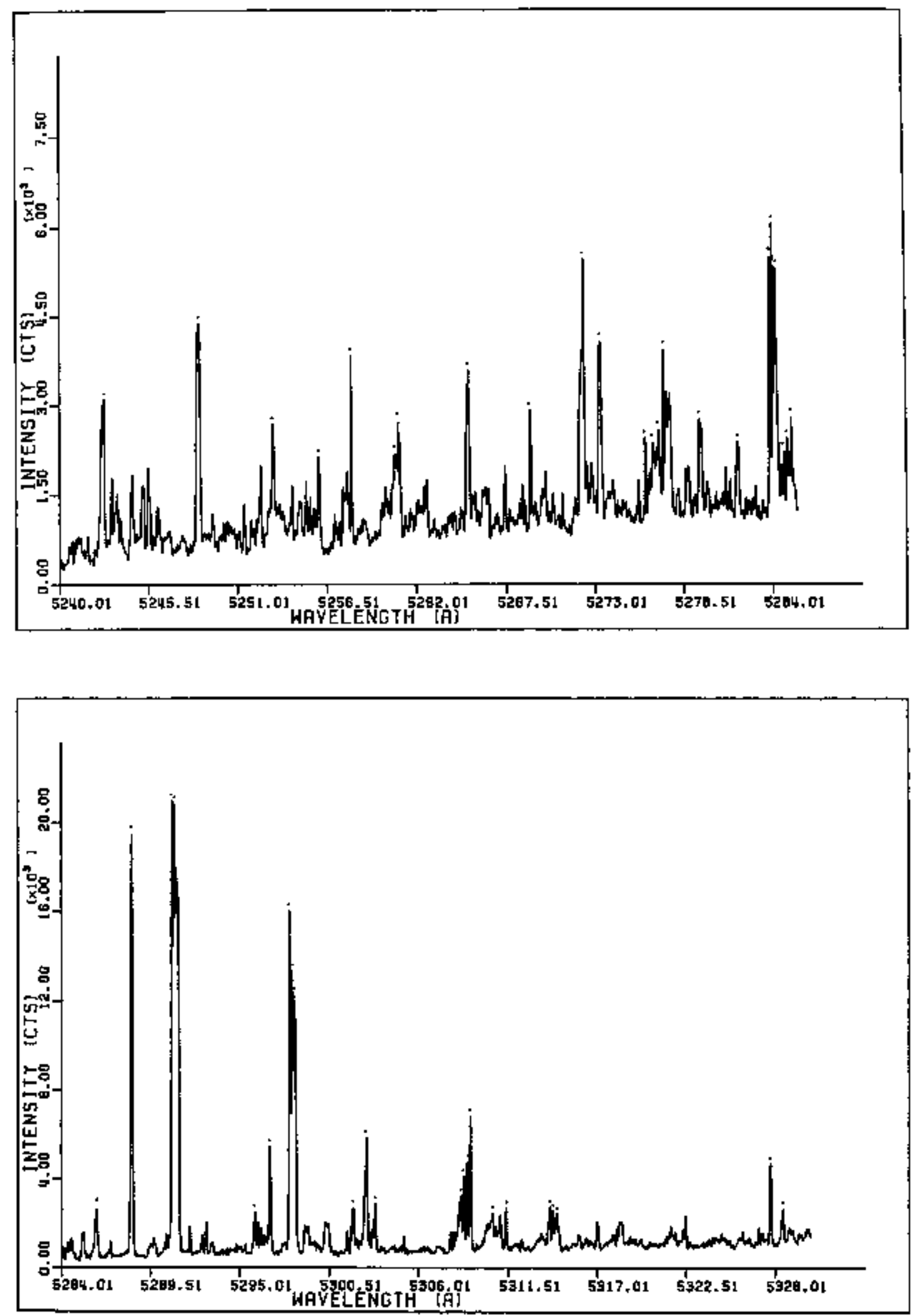


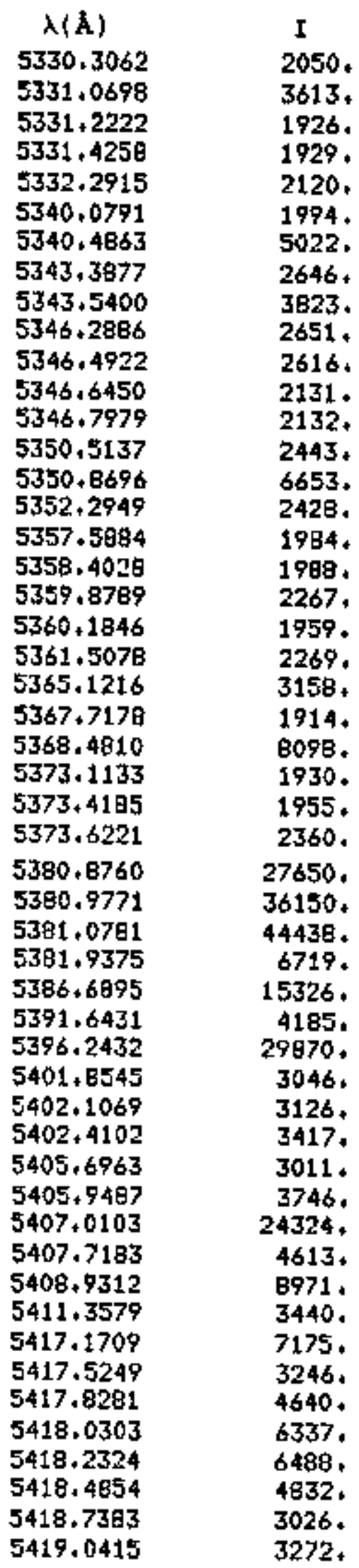



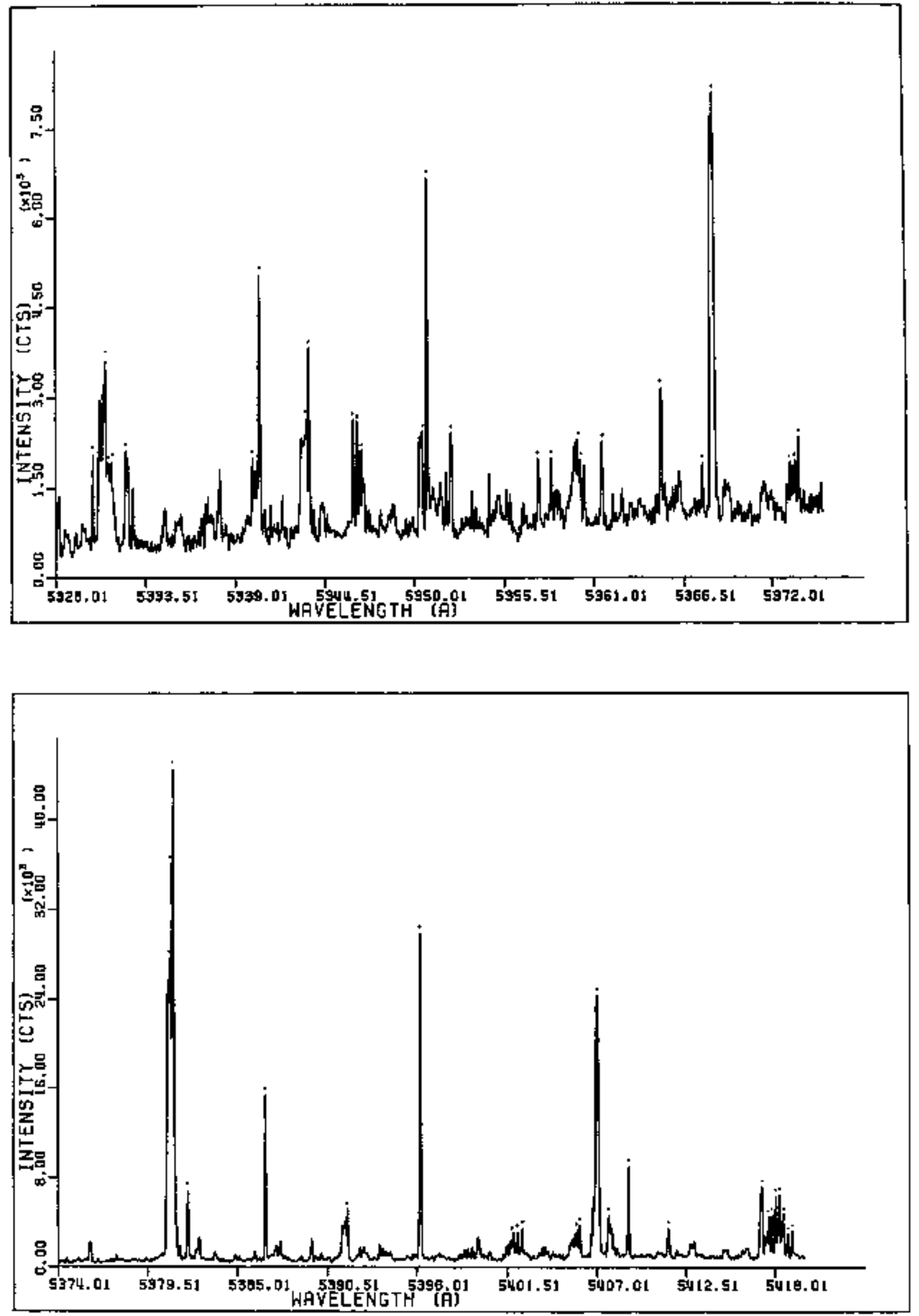


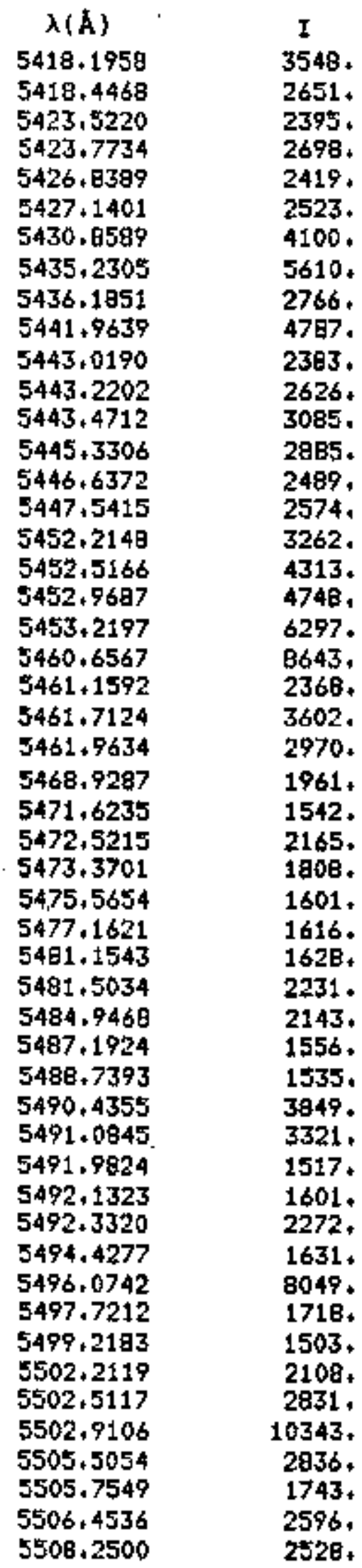



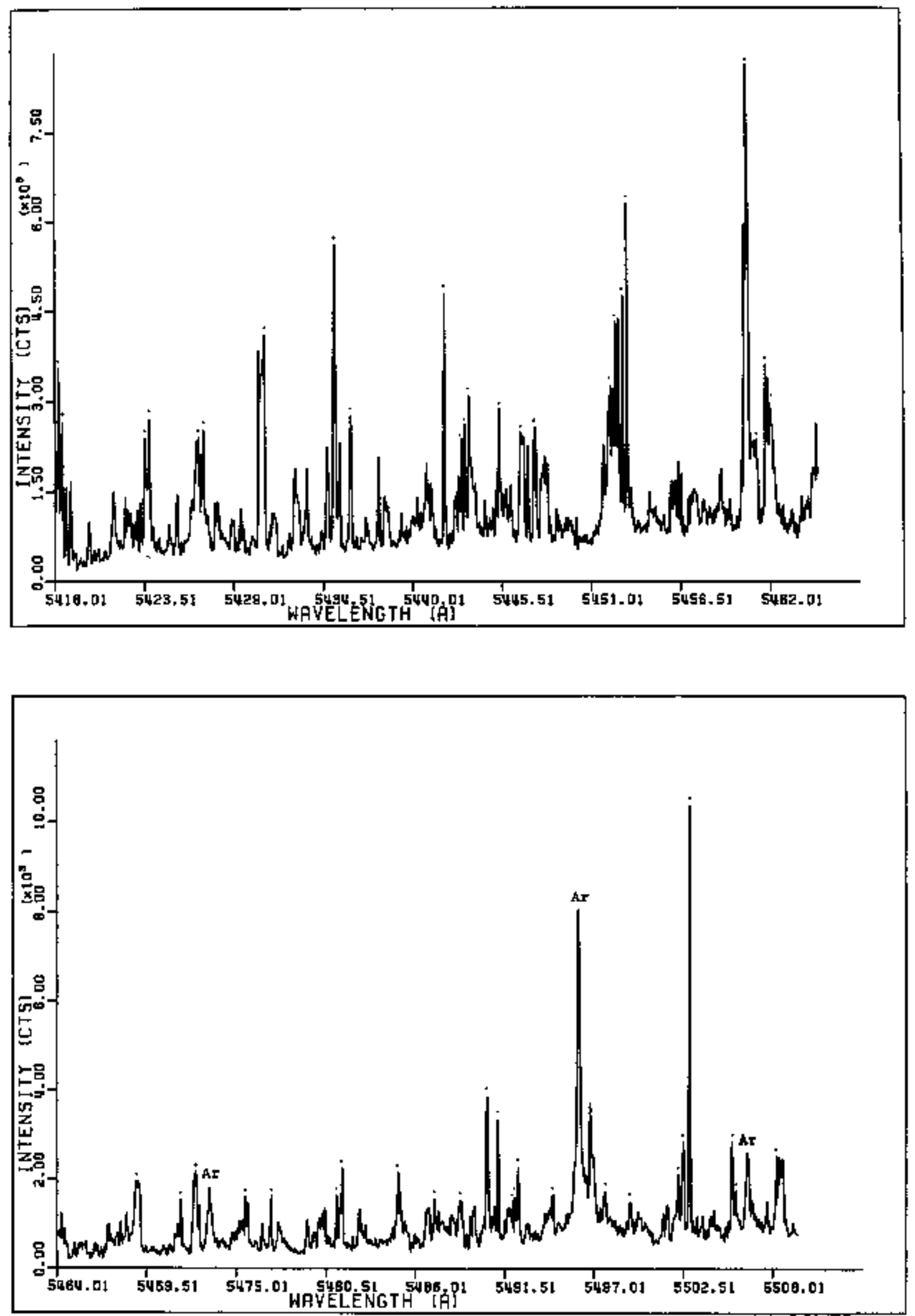


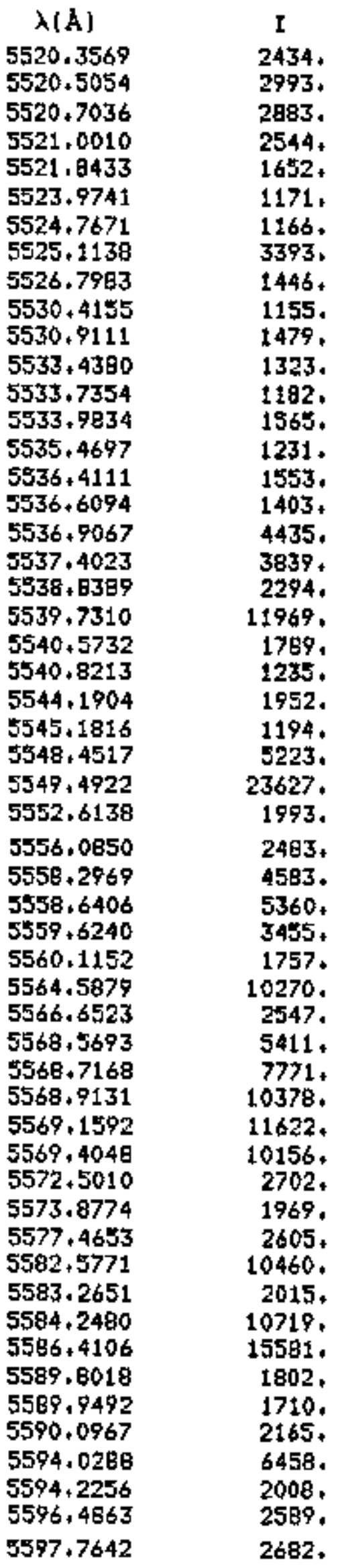



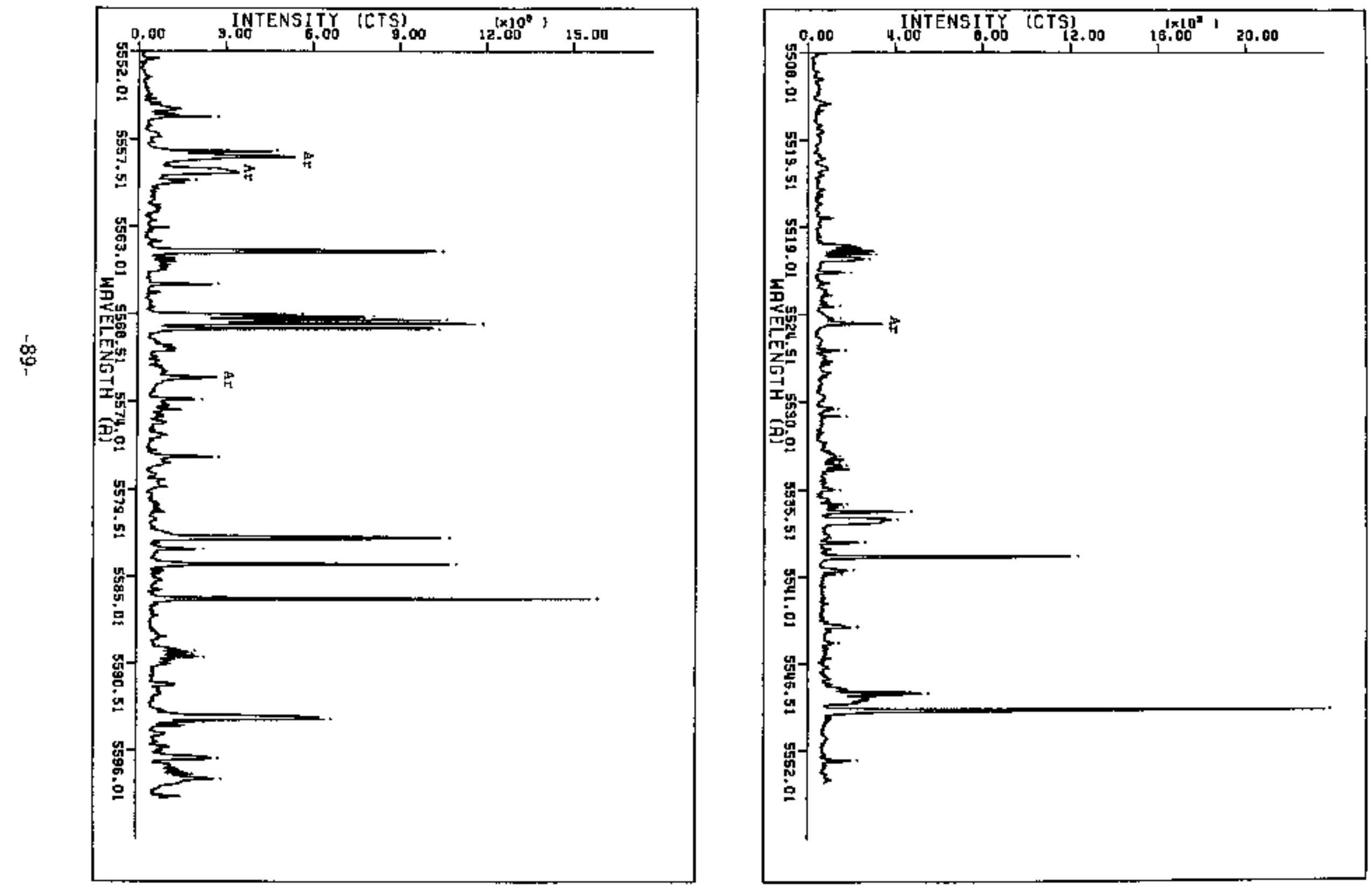


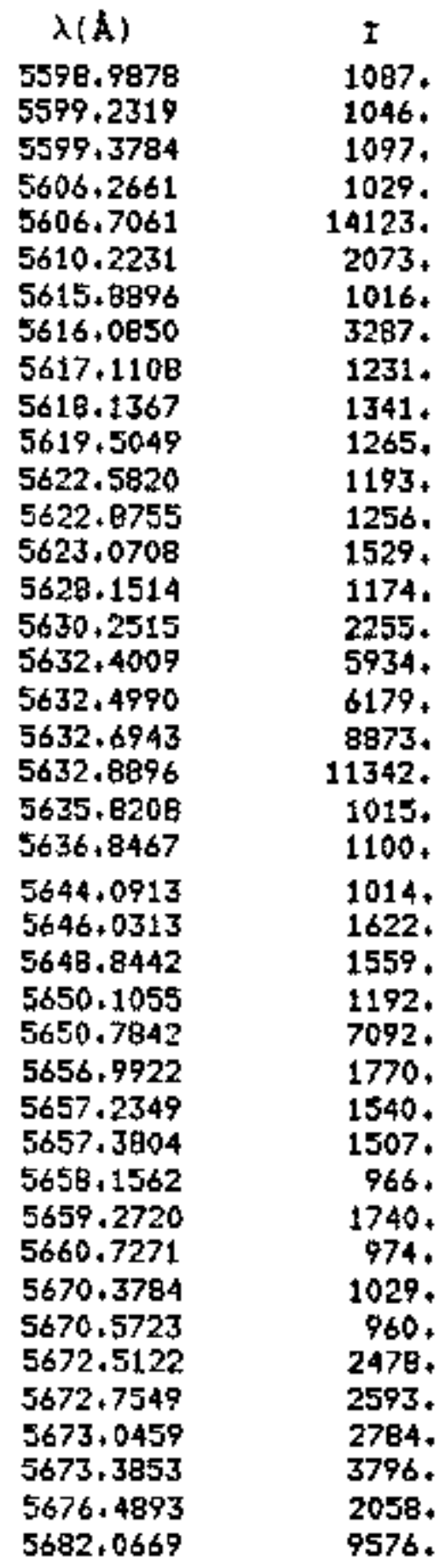



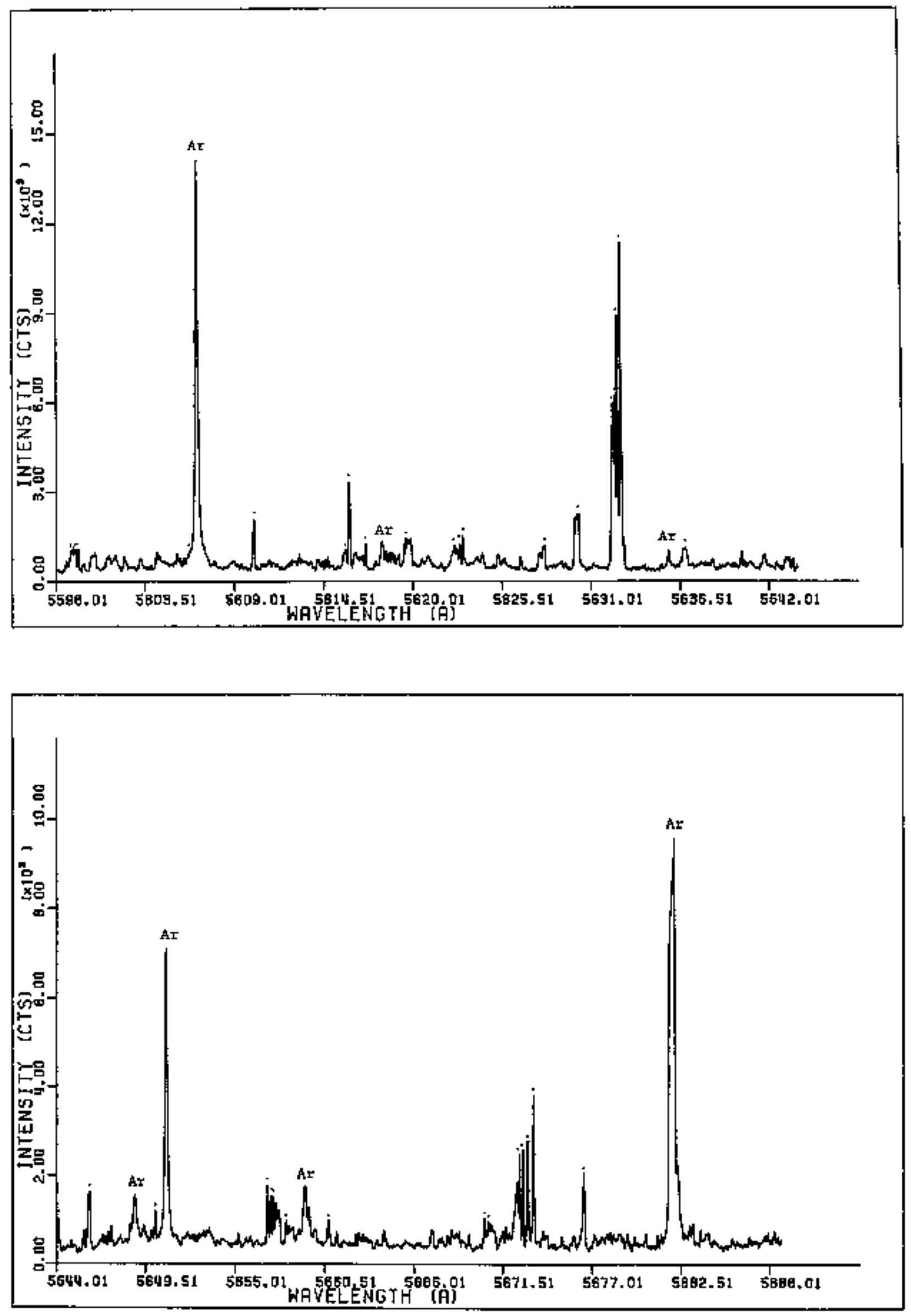


\begin{tabular}{|c|c|}
\hline$\lambda(A)$ & I \\
\hline $\begin{array}{l}5689+9008 \\
5690.5229 \\
5695.9644 \\
5700.2495 \\
5700.5396\end{array}$ & $\begin{array}{r}1657 . \\
874 \\
961 . \\
822 . \\
1393 .\end{array}$ \\
\hline $\begin{array}{l}5700.7310 \\
5700.9233 \\
5706+0757 \\
5709.2534 \\
5710.3608 \\
5712+2871\end{array}$ & $\begin{array}{r}857 . \\
1079 . \\
2116 . \\
968 . \\
1059 . \\
832 .\end{array}$ \\
\hline $\begin{array}{l}5712.8164 \\
5713.8276 \\
5714.4536 \\
5714.9351 \\
5715.2241 \\
5718.6909\end{array}$ & $\begin{array}{r}922 . \\
4706 . \\
963 . \\
1033 . \\
888 . \\
1948 .\end{array}$ \\
\hline $\begin{array}{l}5722+6392 \\
5728.3691\end{array}$ & $\begin{array}{l}849 . \\
920\end{array}$ \\
\hline $\begin{array}{l}5738.5815 \\
5739,7275 \\
5740,3960 \\
5744,4072 \\
5744.6460\end{array}$ & $\begin{array}{l}1147 . \\
5820, \\
1884 . \\
1413 . \\
1224 .\end{array}$ \\
\hline $\begin{array}{l}5744+8369 \\
5744.9800 \\
5745+1235\end{array}$ & $\begin{array}{r}1129 . \\
1025 . \\
966 .\end{array}$ \\
\hline $\begin{array}{l}5745.4575 \\
5745.6963 \\
5754.6255\end{array}$ & $\begin{array}{r}1028 . \\
974 . \\
1146 .\end{array}$ \\
\hline $\begin{array}{l}5757.1562 \\
5757.5386 \\
5758.7319\end{array}$ & $\begin{array}{l}1402 . \\
1124 . \\
1450 .\end{array}$ \\
\hline $\begin{array}{l}5772,5317 \\
5776,0654\end{array}$ & $\begin{array}{l}2242 . \\
2440\end{array}$ \\
\hline
\end{tabular}



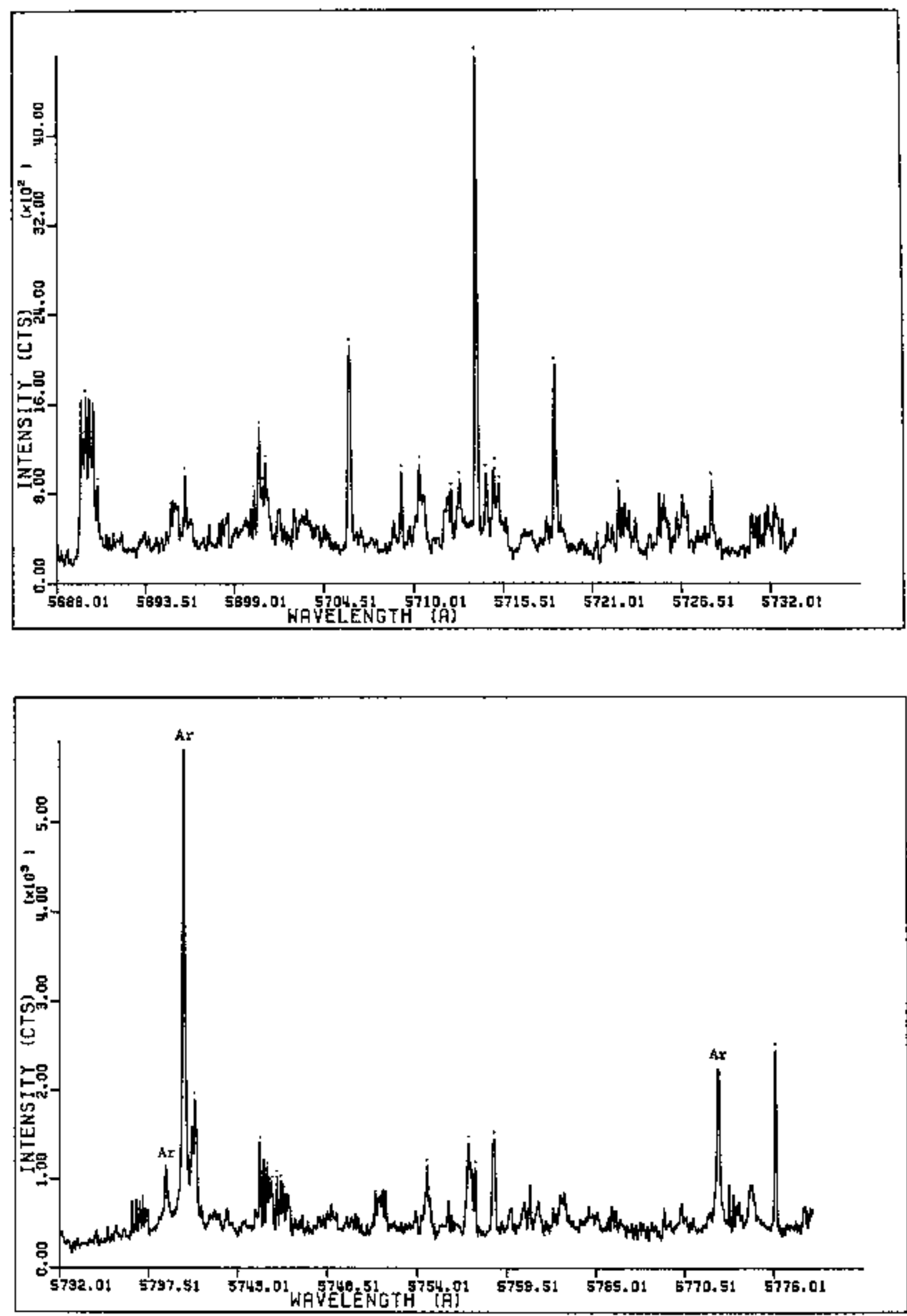

-93- 


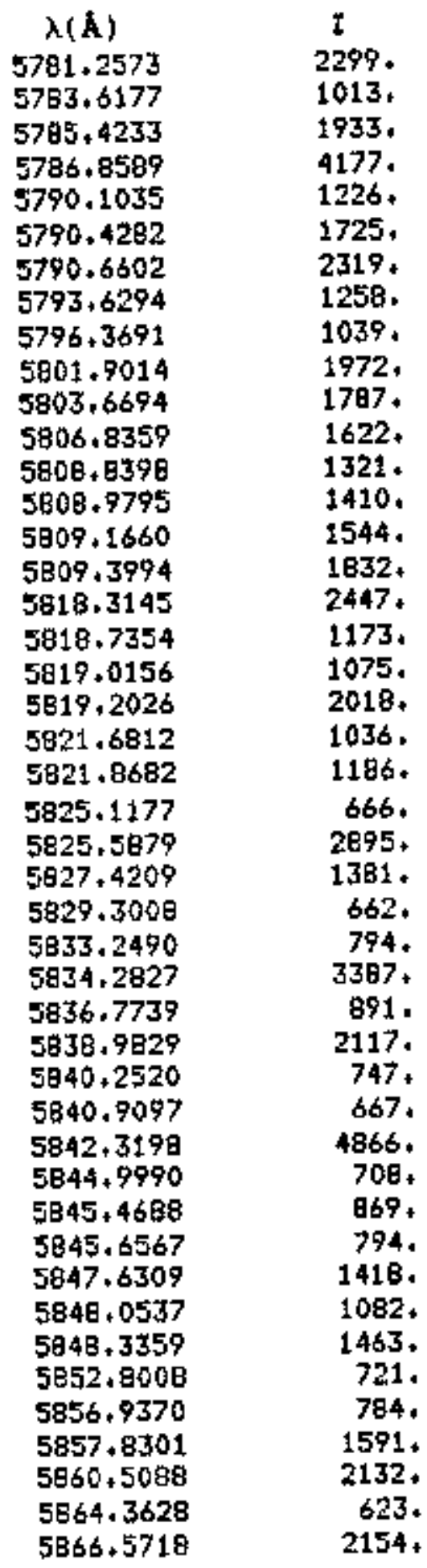



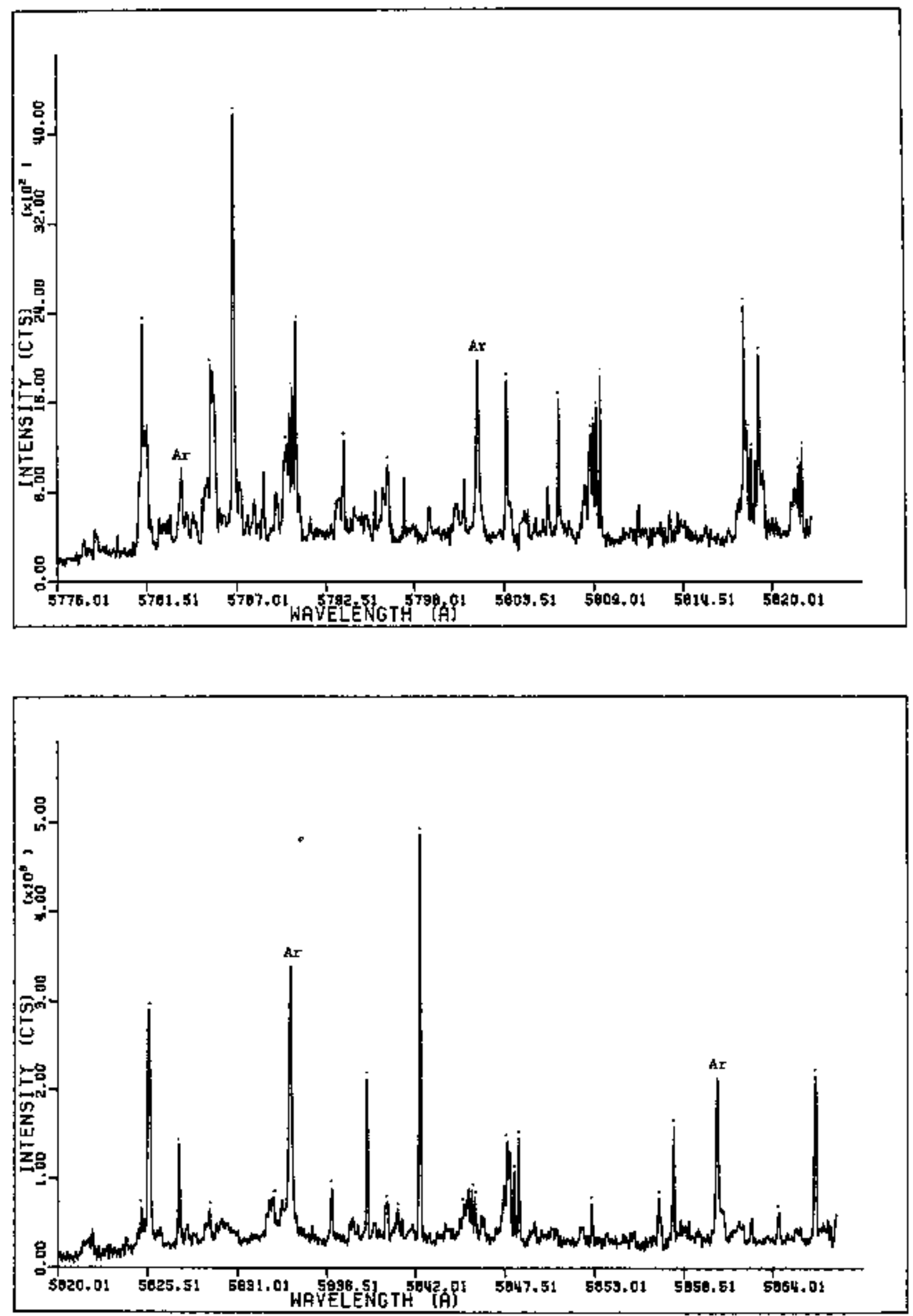


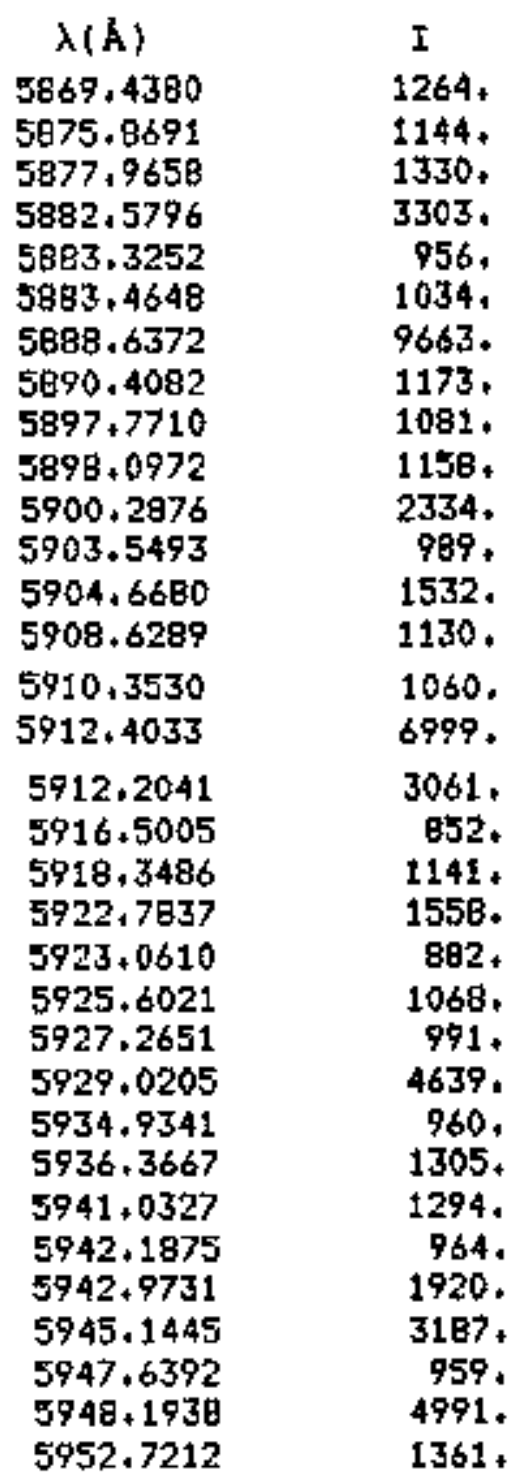



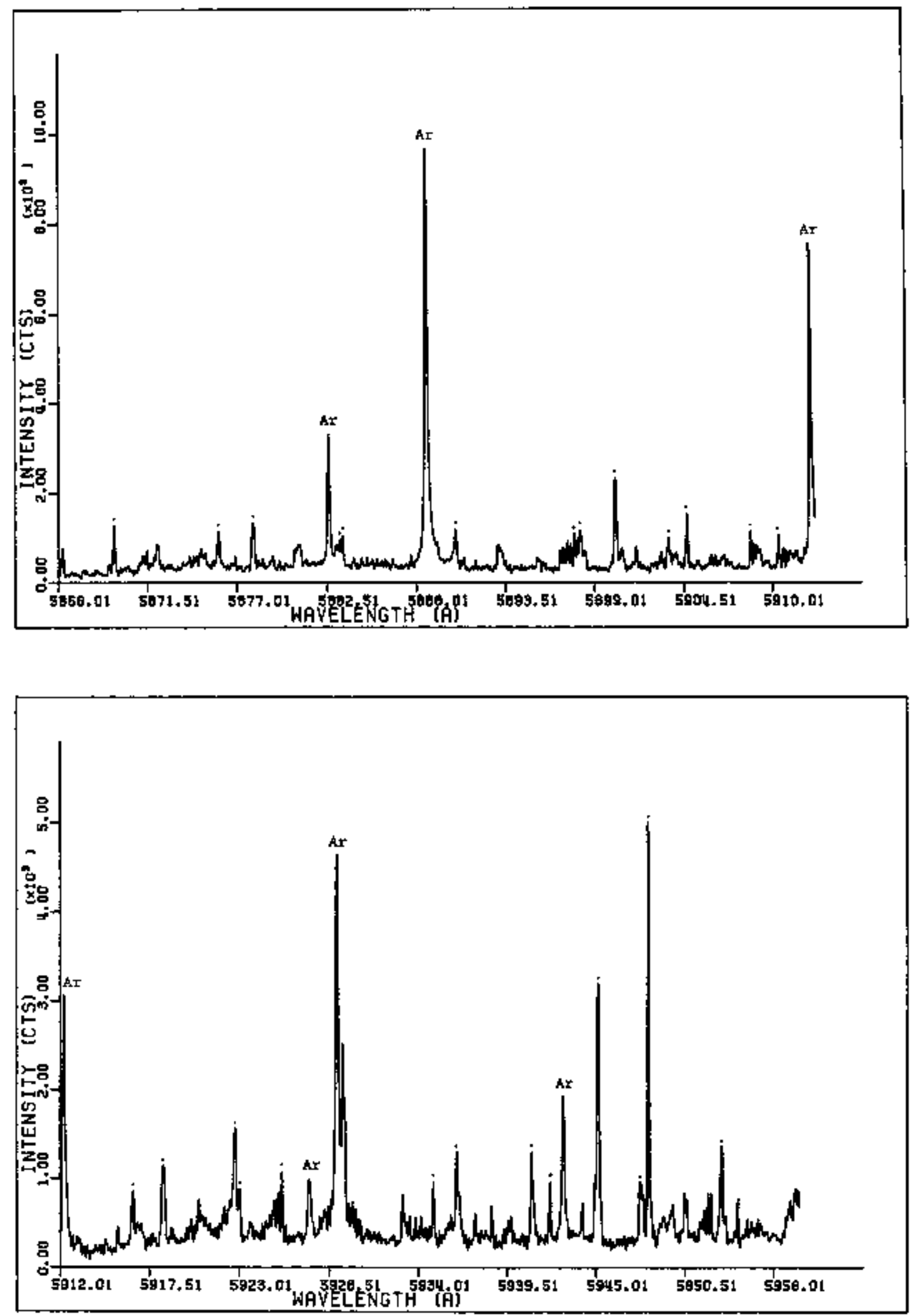

$-97$ 


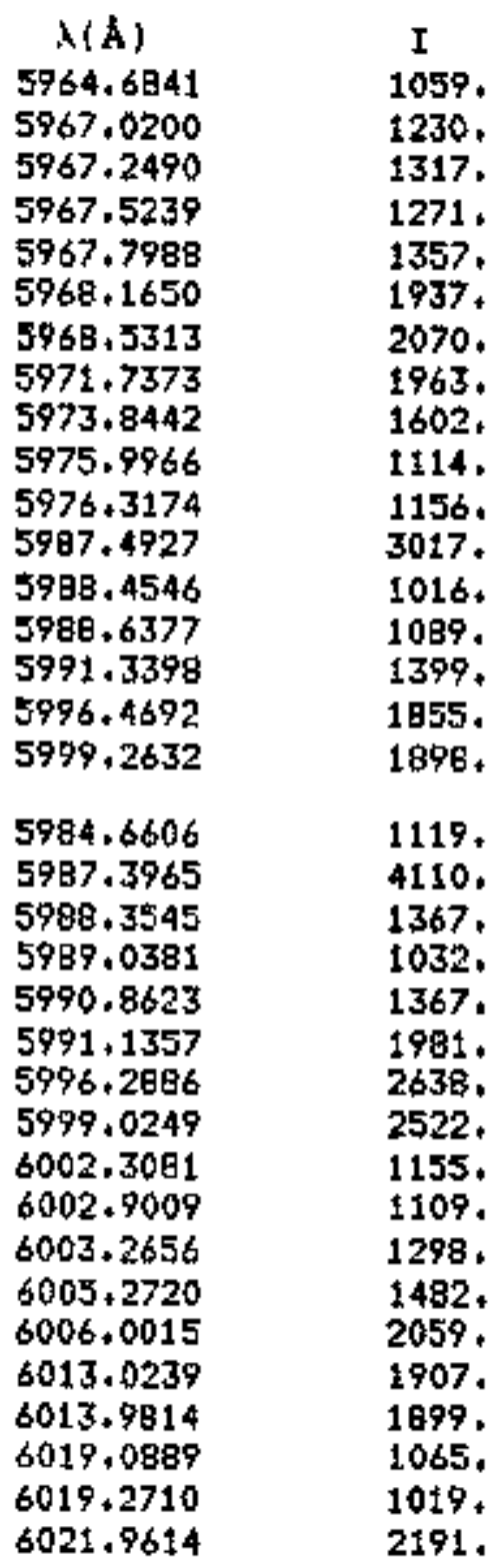



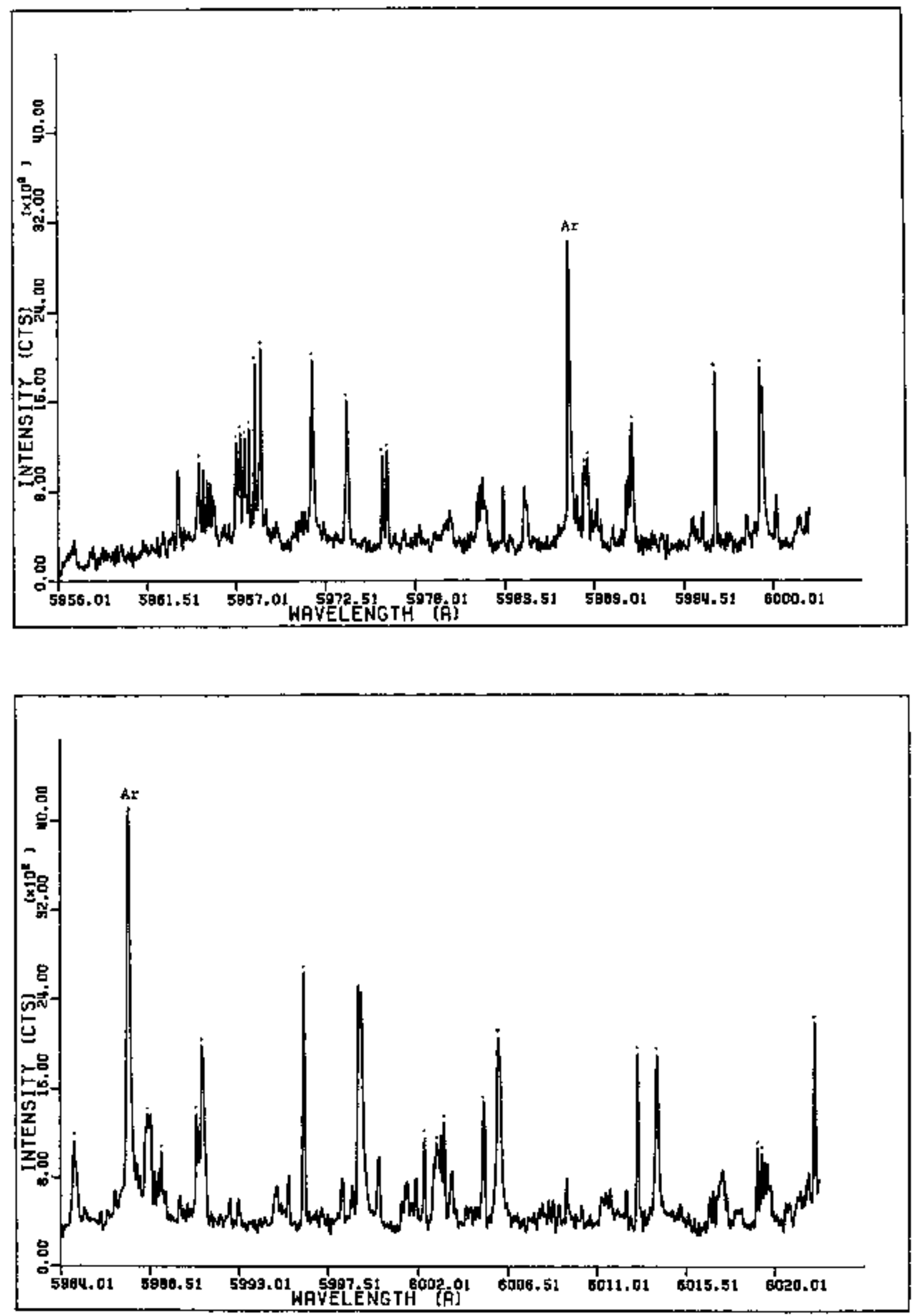


$\begin{array}{cc}\lambda(A) & I \\ 6022 . B 957 & 2265 . \\ 6023.2031 & 1687 . \\ 6023.4297 & 1407 . \\ 6023.6108 & 1145 . \\ 6025.1509 & 4375 . \\ 6032.1270 & 49236 . \\ 6041.3682 & 1280 . \\ 6043.3613 & 20067 . \\ 6046.6685 & 1480 . \\ 6051.6060 & 1265 . \\ 6051.7871 & 1662 . \\ 6052.0137 & 1494 . \\ 6052.3311 & 1646 . \\ 6052.6479 & 2153 . \\ 6052.9197 & 3965 . \\ 6056.5894 & 2219 . \\ 6056.0020 & 1267 . \\ 6056.3616 & 2024 . \\ 6059.3735 & 7345 . \\ 6064.9023 & 1739 . \\ 6074.2520 & 2313 . \\ 6076.8140 & 1301 . \\ 6077.3984 & 1990 . \\ 6092,4775 & 1052 . \\ 6082+7021 & 1450 . \\ 6082.9722 & 1605 . \\ 6083.3315 & 1647 . \\ 6091.1528 & 1901 .\end{array}$



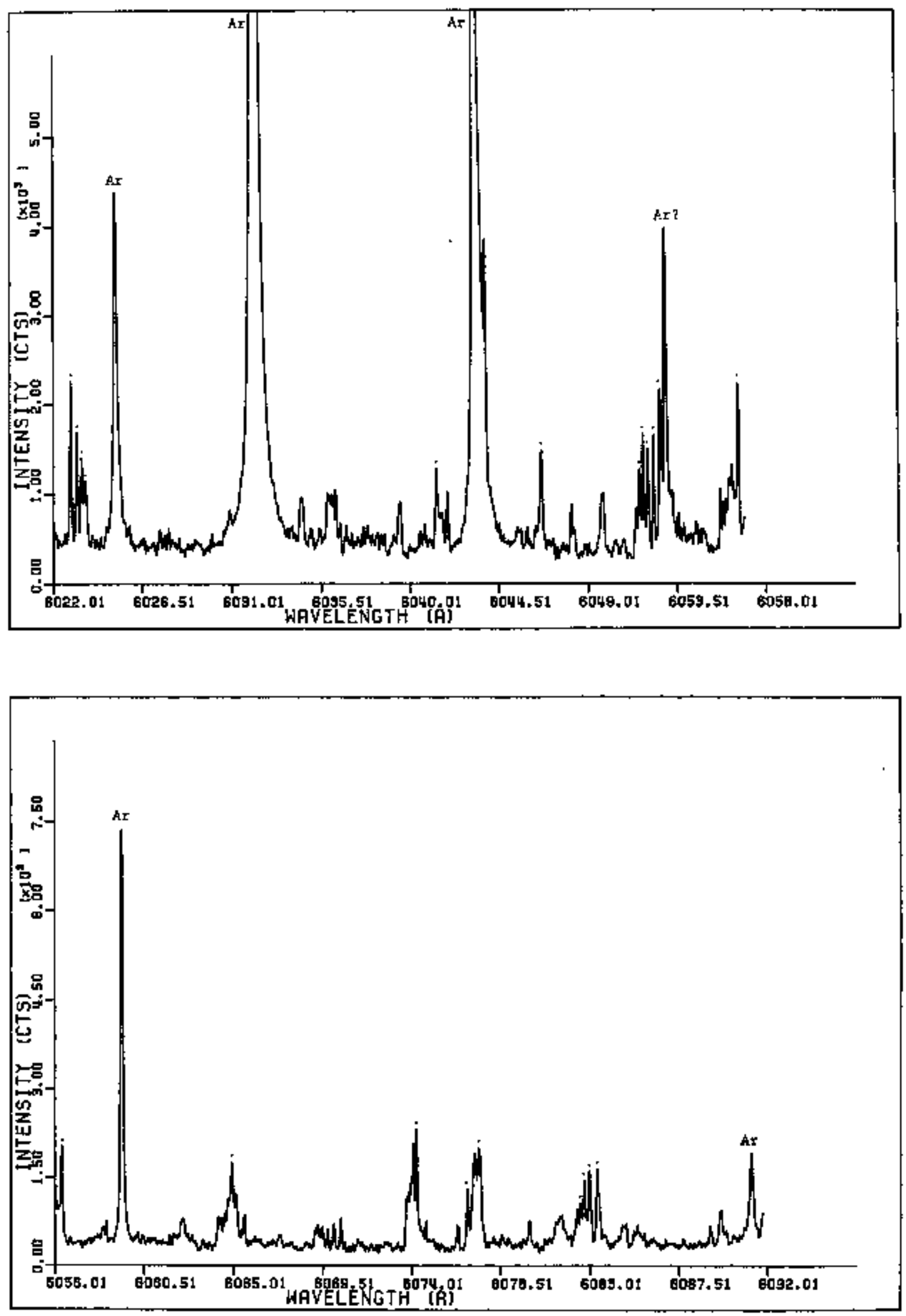


\begin{tabular}{|c|c|}
\hline$\lambda(A)$ & I \\
\hline $\begin{array}{l}6090+7837 \\
6096,8564 \\
6101+2202 \\
6102.3350 \\
6104.2974 \\
6104.6543\end{array}$ & $\begin{array}{l}1862, \\
3127 . \\
1440, \\
5464 . \\
1187 . \\
1654 .\end{array}$ \\
\hline $\begin{array}{l}6105+6802 \\
6106.4829 \\
6106+7505 \\
6107.5532\end{array}$ & $\begin{array}{l}7571 \\
1065 \\
1051 . \\
1134 .\end{array}$ \\
\hline $\begin{array}{l}6111.3442 \\
6112+9946\end{array}$ & $\begin{array}{l}7231 . \\
1000\end{array}$ \\
\hline 6113,6187 & 1125 \\
\hline $\begin{array}{l}6120.5767 \\
6123.2969\end{array}$ & $\begin{array}{l}2155 . \\
1066 .\end{array}$ \\
\hline 6123,6530 & 1134 \\
\hline $\begin{array}{l}6123.7876 \\
6123.9214\end{array}$ & $\begin{array}{l}1372 . \\
1162 .\end{array}$ \\
\hline $\begin{array}{l}6124+2783 \\
6124.9028\end{array}$ & $\begin{array}{l}1212+ \\
1228 .\end{array}$ \\
\hline $\begin{array}{l}6127+4790 \\
6128+8066 \\
6129,1162\end{array}$ & $\begin{array}{l}109 \% . \\
1034 . \\
1407 .\end{array}$ \\
\hline $\begin{array}{l}6132+5679 \\
6139 \cdot 0728 \\
6139+3923\end{array}$ & $\begin{array}{l}1344 \text {. } \\
1189 . \\
1387 .\end{array}$ \\
\hline $\begin{array}{l}6145.6216 \\
6155.5337 \\
6156.2417\end{array}$ & $\begin{array}{l}6363 . \\
2157 . \\
3312 .\end{array}$ \\
\hline $\begin{array}{l}6157.0381 \\
6158.4985\end{array}$ & $\begin{array}{l}5070 . \\
7056 .\end{array}$ \\
\hline
\end{tabular}



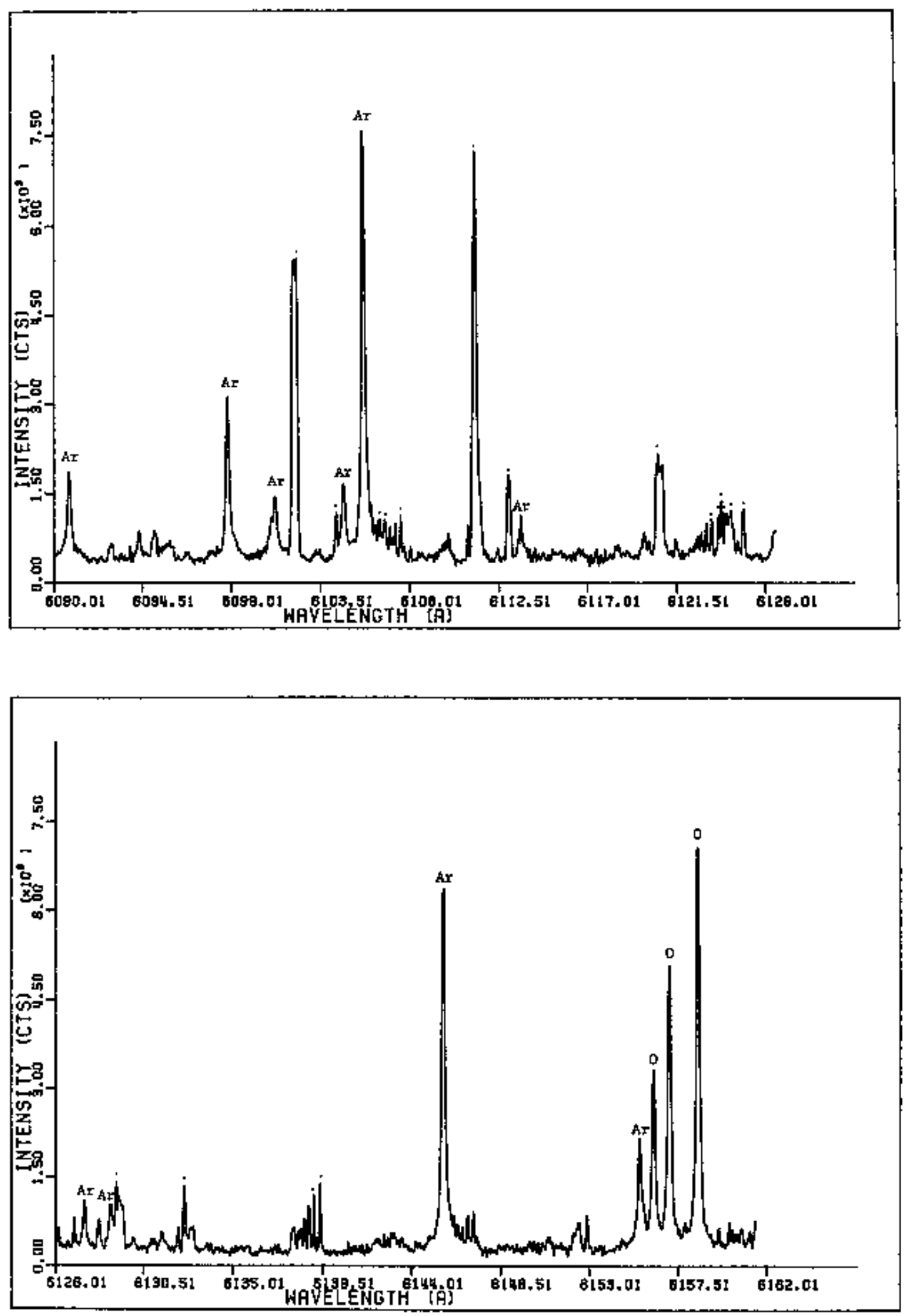


$\begin{array}{cc}\lambda(A) & I \\ 6163.5278 & 1227 . \\ 6163.6592 & 1120 . \\ 6165.0640 & 1283, \\ 6167.5664 & 124 \mathrm{.} . \\ 6170+1567 & 4265 . \\ 6172.2637 & 1099 . \\ 6172.6597 & 1202 . \\ 6173.0981 & 5374 . \\ 6191.0967 & 1214 . \\ 6191.5361 & 1265 . \\ 6194+2578 & 1136 . \\ 6194.7647 & 2337 . \\ 6194.9600 & 1114 . \\ 6206.5542 & 982 . \\ 6207.2075 & 944 . \\ 6211.7363 & 930 . \\ 6212.4331 & 5072 . \\ 6213.8271 & 1025 . \\ 6214.0884 & 1029 . \\ 6215.8735 & 4385 . \\ 6218.9658 & 1070 . \\ 6222.6675 & 957 . \\ 6226.1953 & 888 .\end{array}$



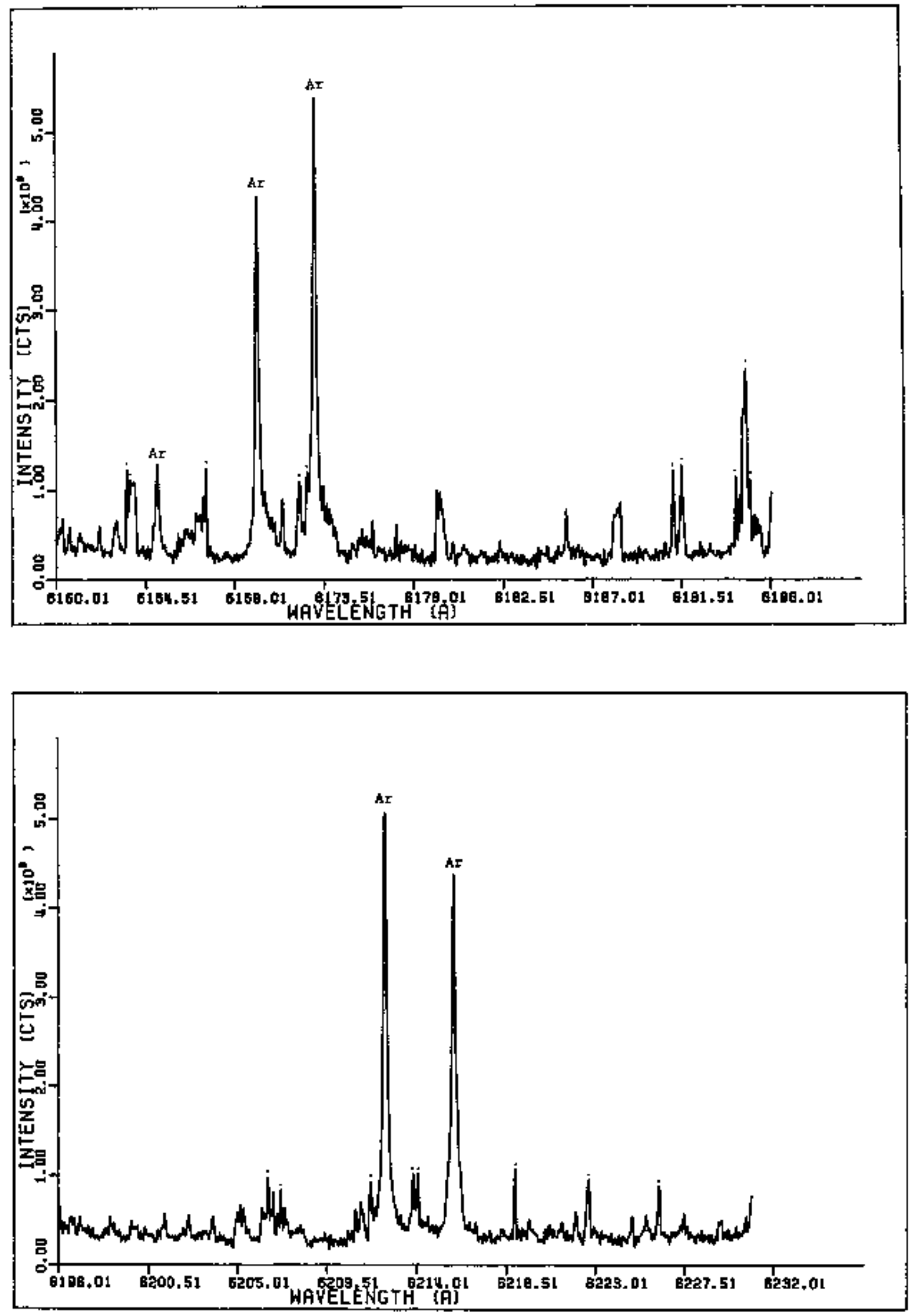


\begin{tabular}{cr}
$\lambda(A)$ & \multicolumn{1}{c}{1} \\
6231.2085 & 645. \\
6232.2437 & 1072. \\
6236.3433 & 862. \\
6236.6885 & 852. \\
6738.4575 & 1695. \\
6243.7217 & 738. \\
6244.0669 & 751. \\
6248.6841 & 1202. \\
6249.4175 & 1447. \\
6257.3574 & 1976. \\
6263.4414 & 827. \\
6263.8296 & 733. \\
6264.0884 & 727. \\
6269.9971 & 915. \\
6273.0889 & 1006. \\
6273.3008 & 666. \\
6275.9687 & 1992. \\
6276.5615 & 1234. \\
6279.3564 & 656. \\
6286.3867 & 1339. \\
6288.8008 & $1964+$ \\
6290.5371 & 955. \\
6297.1436 & 5615.
\end{tabular}



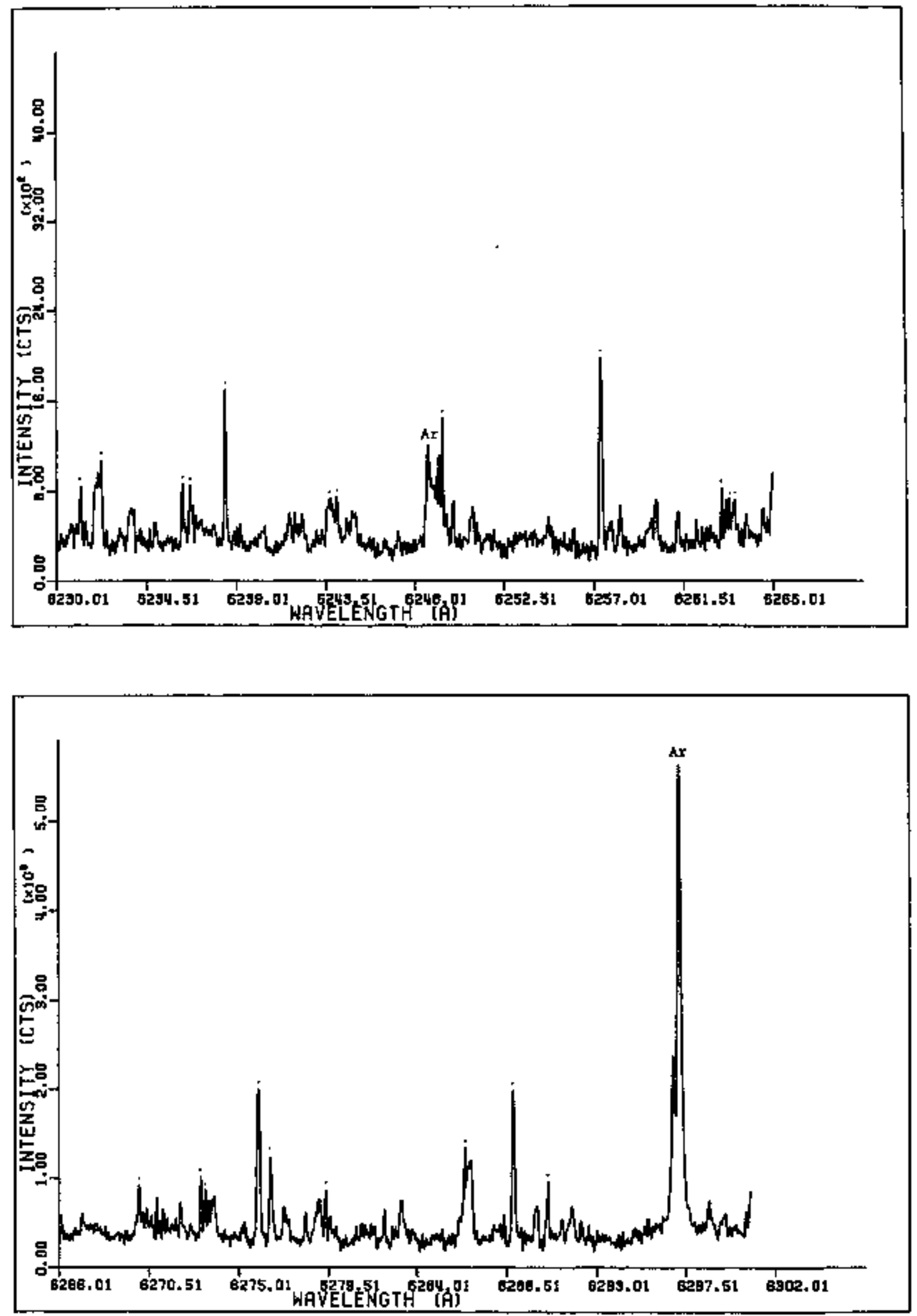


\begin{tabular}{cr}
$\lambda(A)$ & \multicolumn{1}{c}{$I$} \\
6307.6597 & 7567. \\
6309.0591 & 1332. \\
6309.5254 & 911. \\
6313.2988 & 1098. \\
6314.0195 & 813. \\
6316.4790 & 1301. \\
6316.9878 & 1372. \\
6317.4116 & 801. \\
6317.6660 & 956. \\
6317.8779 & 940. \\
6321.0581 & 1275, \\
6323.2632 & 796. \\
6329.6230 & 782. \\
6325.9624 & 1256. \\
6333.9902 & 873. \\
6334.4565 & 916. \\
6334.9653 & 848. \\
6334.1304 & 967. \\
6334.6343 & 937. \\
6337.4482 & 1641. \\
6338.2461 & 925. \\
6352.1484 & 1391, \\
6364.9320 & 1645. \\
6369.5781 & 3337.
\end{tabular}



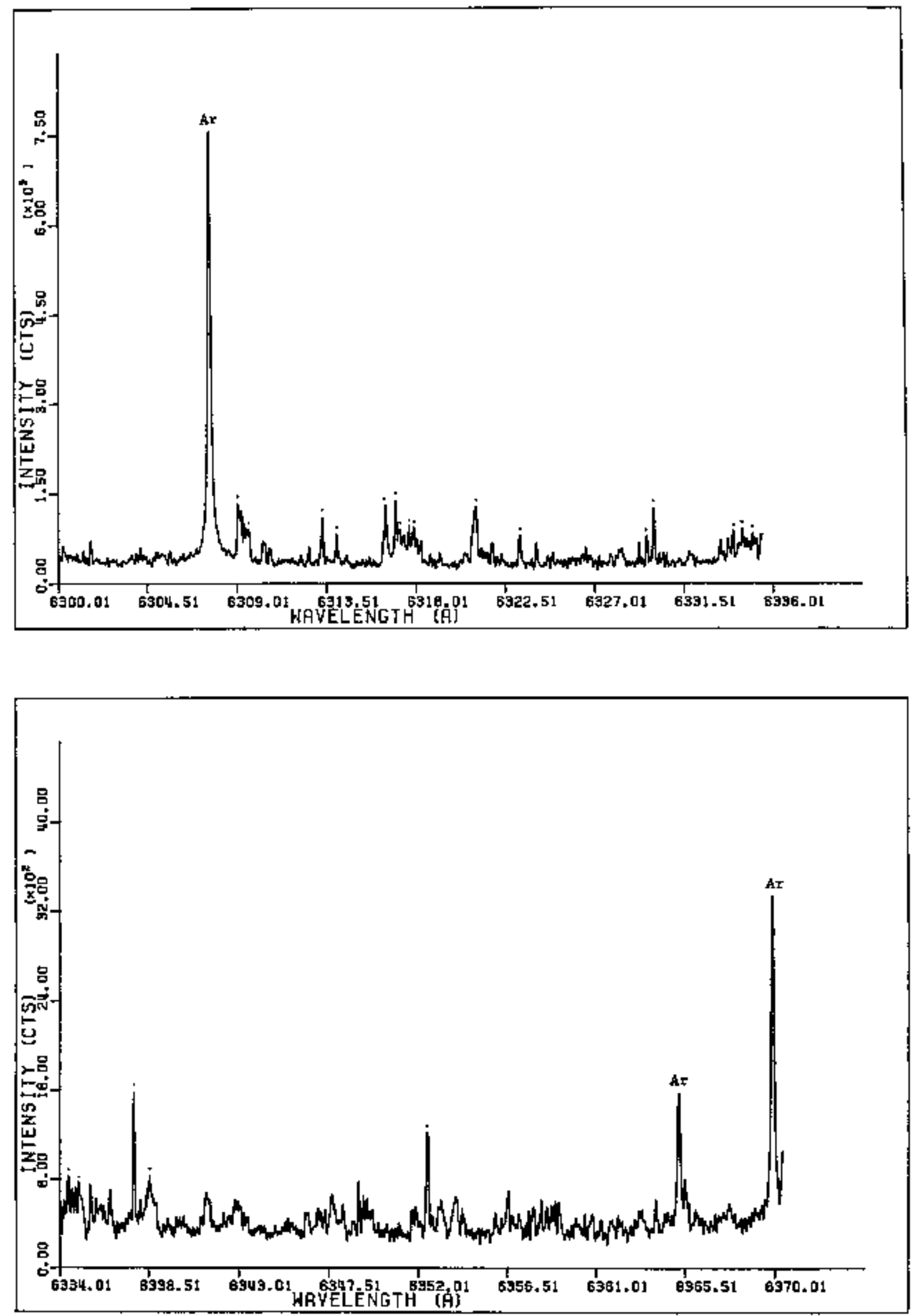


\begin{tabular}{lr}
$\lambda(A)$ & \multicolumn{1}{c}{1} \\
6375.9014 & 869. \\
6377.0654 & 1029. \\
6384.6782 & 5472. \\
6385.3022 & 857. \\
6388.0063 & 1028 \\
6389.7959 & 836. \\
6389,9614 & 877. \\
6396.1602 & 847. \\
6396.3687 & 938. \\
6397.4502 & 1034. \\
6397.7407 & 1134. \\
6400.4033 & 1933. \\
6400.9023 & 866. \\
6403.2319 & 840. \\
6407.6035 & 688 \\
6407.8101 & 602. \\
6408.0161 & 574, \\
6410.2852 & 694. \\
6416.3486 & 22118. \\
6423.4023 & 740. \\
6424.3931 & 974. \\
$6424+6812$ & 1178. \\
$6424+9697$ & 1126. \\
6430.1690 & 807. \\
6431.6934 & 1384. \\
$6433+3022$ & 646.
\end{tabular}



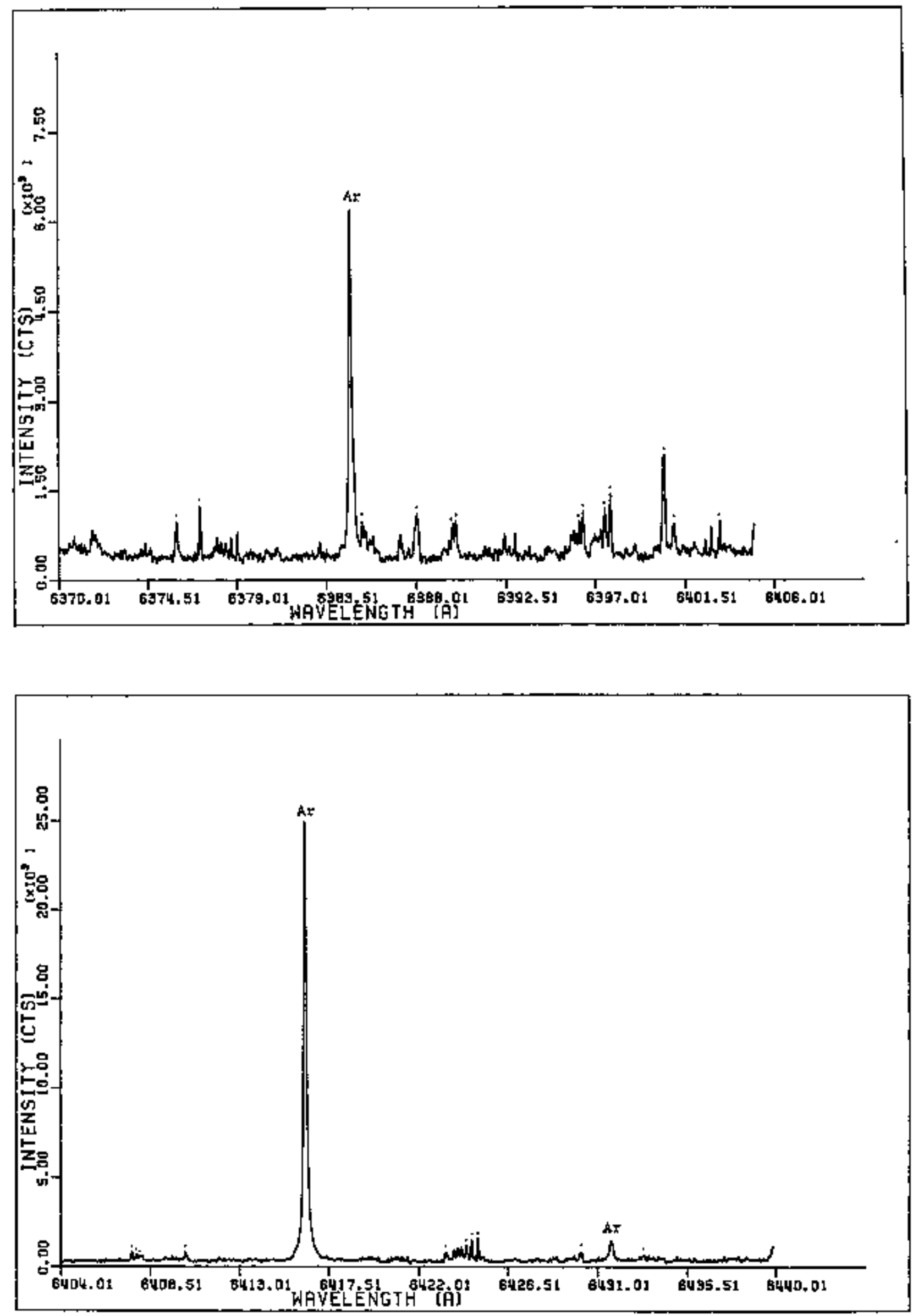


$\begin{array}{lr}\lambda(A) & I \\ 6440+1270 & 567 . \\ 6440.4141 & 792 . \\ 6444.3857 & 977, \\ 6449+7505 & 507 . \\ 6449.9146 & 590 . \\ 6453.6001 & 1092 . \\ 6454.4600 & 1589 . \\ 6455.9751 & 2166 . \\ 6466.7036 & 956 . \\ 6467.6460 & 586 . \\ 6468+1777 & 618 . \\ 6469.3823 & 932 . \\ 6468,9146 & 589 . \\ 6469.2837 & 661 . \\ 6479.6885 & 511 . \\ 6481.3511 & 557 . \\ 6483.0542 & 543 . \\ 6493.7593 & 477 . \\ 6494.1240 & 464 . \\ 6495.2188 & 400 . \\ 6495.3809 & 466 . \\ 6499.6392 & 416 . \\ 6500.0439 & 467 . \\ 6507.6270 & 397 . \\ 6509.2896 & 548 .\end{array}$



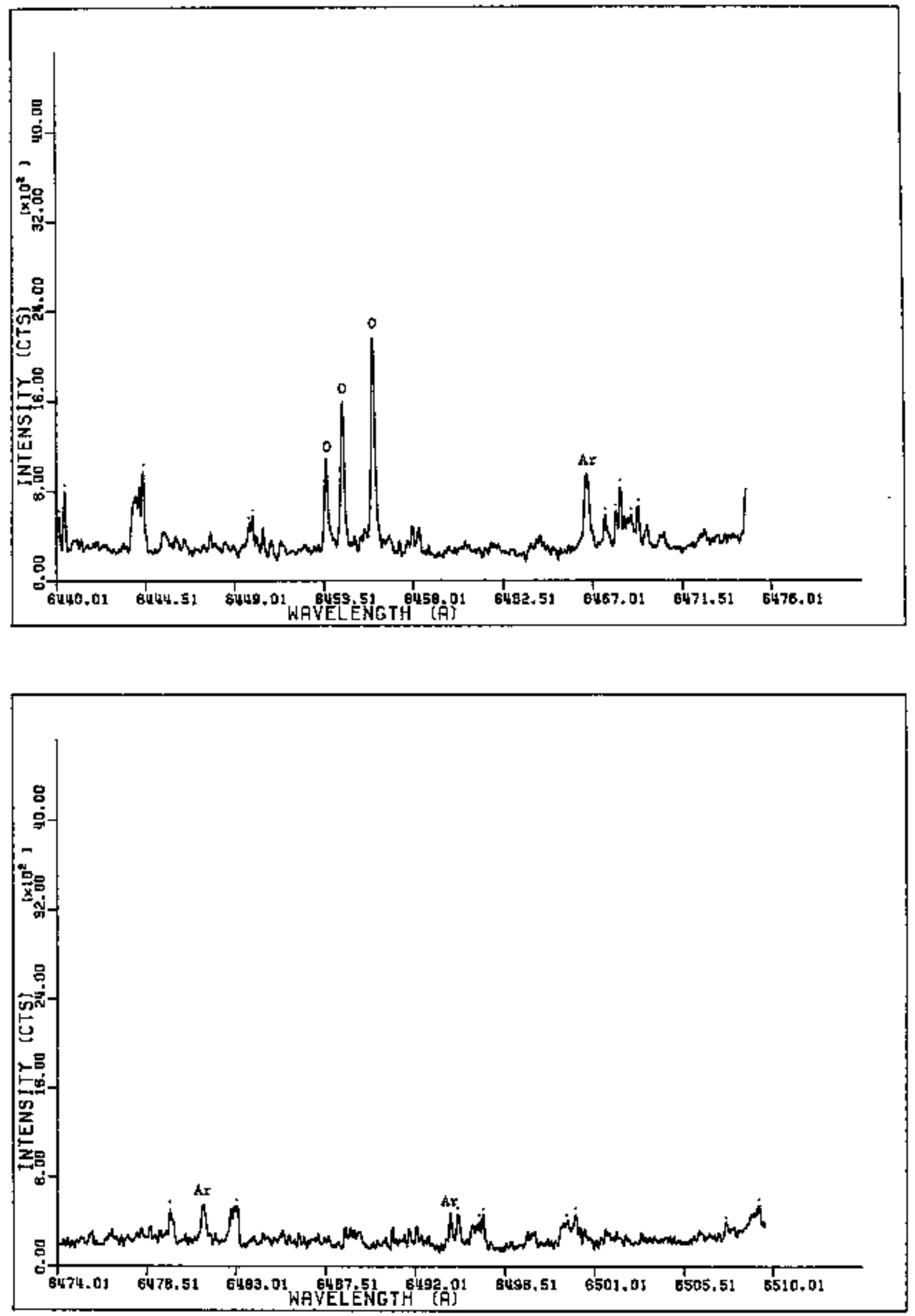


\begin{tabular}{lr}
$\lambda(\AA)$ & \multicolumn{1}{c}{$I$} \\
6508.8477 & 445. \\
6513.7925 & 497, \\
6515.7222 & 405. \\
6515.9634 & 394. \\
6517.4102 & 462. \\
6520.7471 & 402. \\
6521.2695 & 1697. \\
6530.3145 & 564. \\
6531.5210 & 453. \\
$6538+1138$ & 2361. \\
$6540+2036$ & 441 \\
6538.0737 & 2425. \\
6558.9019 & 1048. \\
6559.8989 & 1123. \\
6562.8521 & 226941. \\
6566.0840 & 1211. \\
6568.7173 & 1019.
\end{tabular}



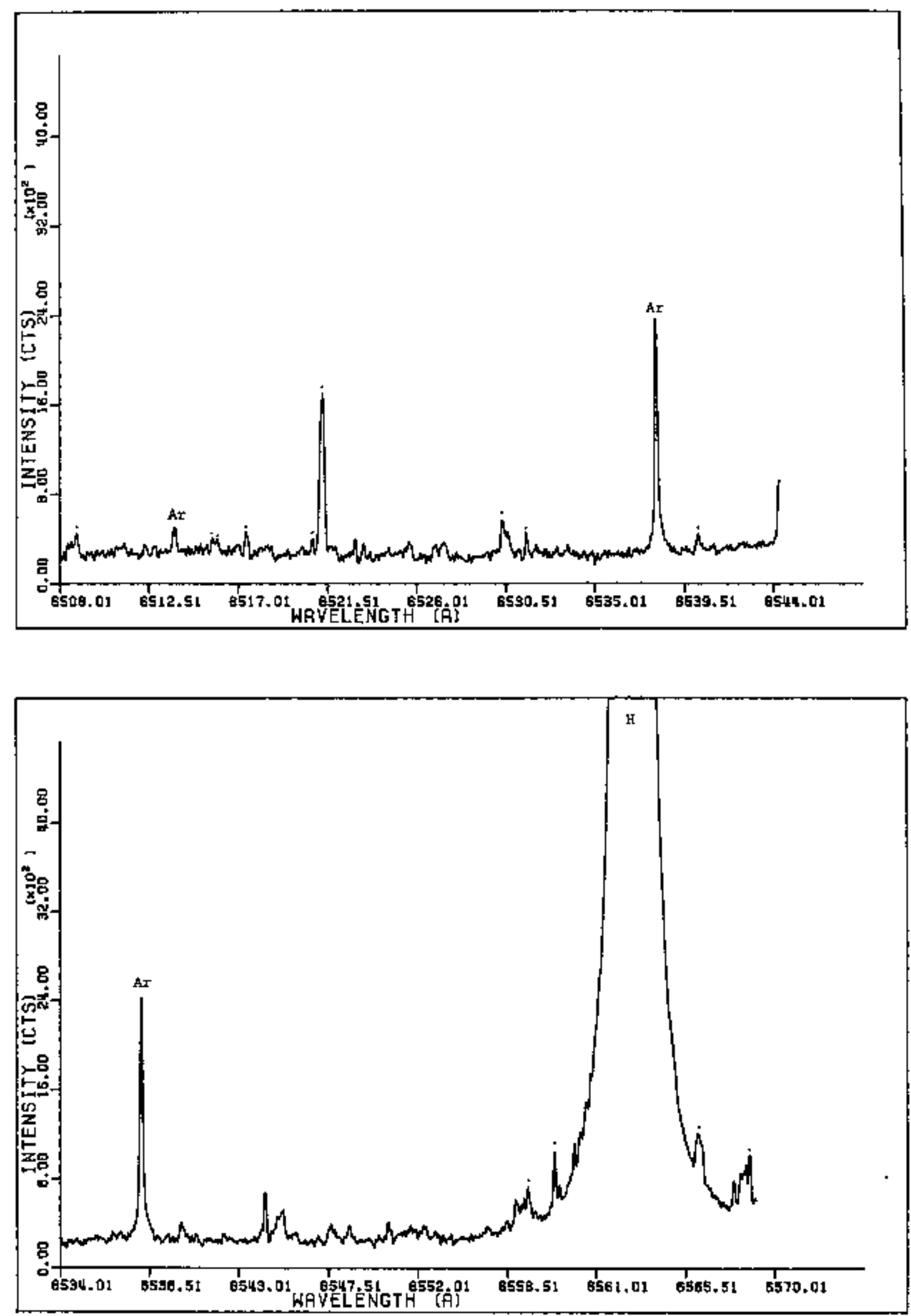


$\begin{array}{cc}\lambda(A) & I \\ 6569.3384 & 381 . \\ 6569.4966 & 370 . \\ 6570.9804 & 407 . \\ 6571.4731 & 367 . \\ 6572.0669 & 385 . \\ 6585.0000 & 370 . \\ 6590.2202 & 410 . \\ 6592.0005 & 633 . \\ 6593.9775 & 525 . \\ 6594.8086 & 804 . \\ 6596.3105 & 500 . \\ 6598.8174 & 572 . \\ 6602.6191 & 459 . \\ 6604+8936 & 4360 . \\ 6605.7168 & 467 . \\ 6606.1475 & 508 . \\ 6612.9292 & 959 . \\ 6618.8877 & 635 . \\ 6626.9233 & 702 .\end{array}$



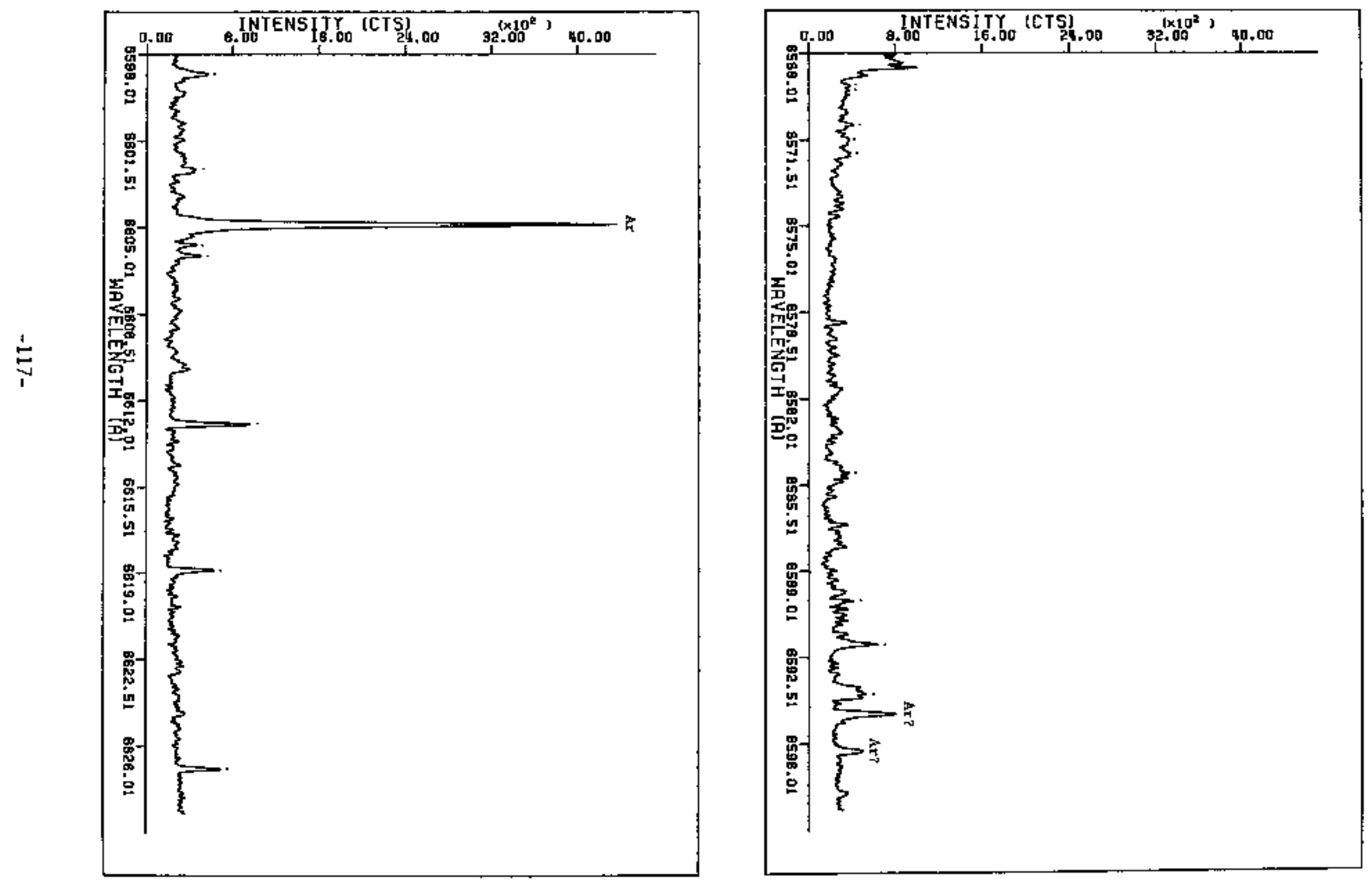


\begin{tabular}{lr}
$\lambda(A)$ & \multicolumn{1}{c}{$I$} \\
6632.3159 & 545. \\
6637.3213 & 1209. \\
6637.5933 & 891. \\
6638.3301 & 467. \\
$6638+6401$ & 462. \\
6638.7959 & 505. \\
6646.3228 & 530. \\
6649.1167 & 538. \\
$6649+3101$ & 612. \\
6651.2114 & 472. \\
$6653+3848$ & 481. \\
6657.3032 & 487. \\
6657.8853 & 501. \\
6658.0796 & 478. \\
6658.2729 & 544. \\
6660.7090 & 2199. \\
$6661+1318$ & 541. \\
6664.0542 & 2413. \\
$6669+4756$ & 1060. \\
6677.2913 & 9716. \\
6678.7908 & 506. \\
6678.9346 & 538, \\
$6684+9321$ & 654. \\
6686.2017 & 583.
\end{tabular}



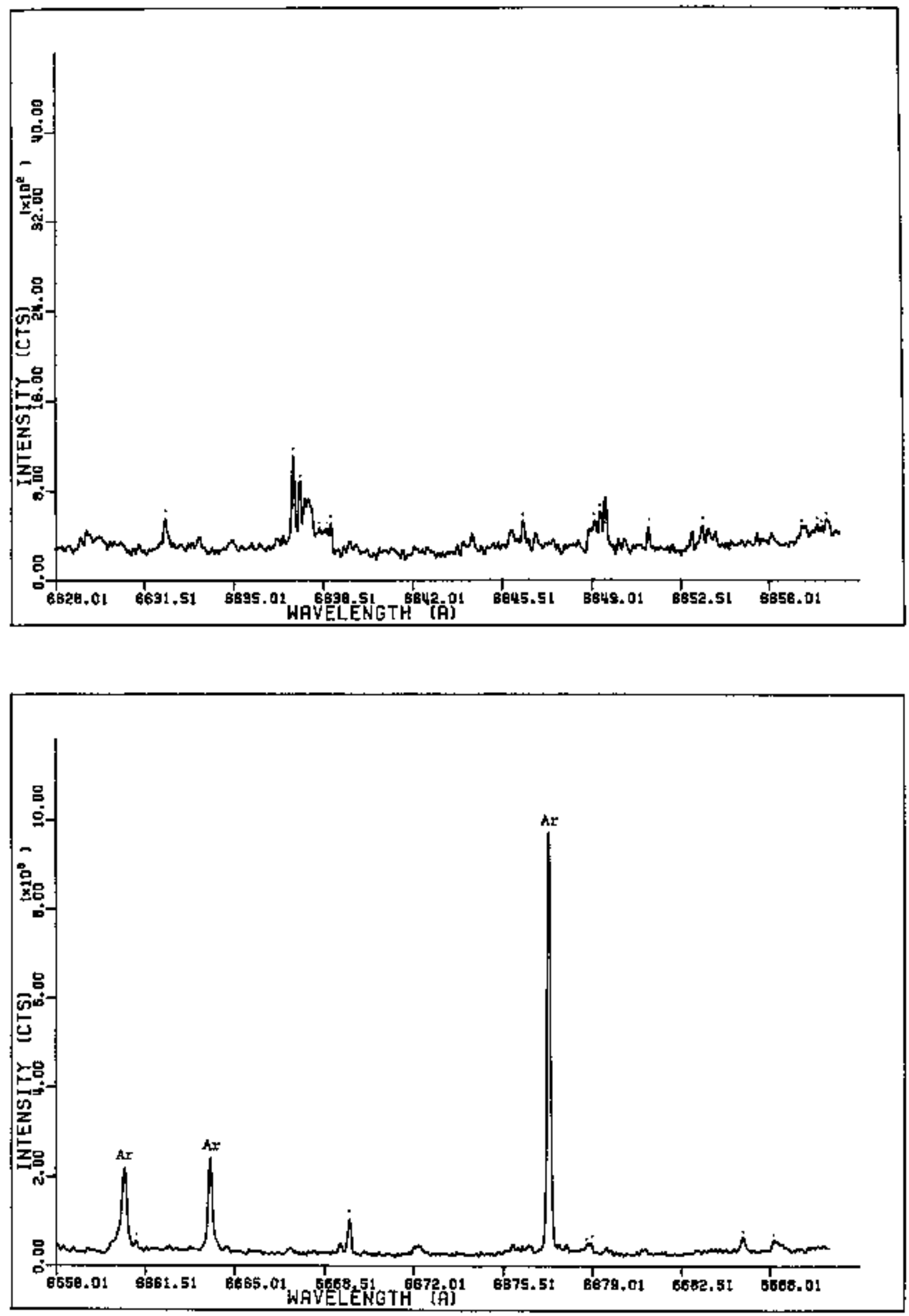


\begin{tabular}{cr}
$\lambda(A)$ & \multicolumn{1}{c}{$I$} \\
6689.3438 & 1369. \\
6690.7149 & 1927. \\
6694.2202 & 2466. \\
6699.0977 & 1066. \\
6699.3213 & 1082. \\
6704.5073 & 606. \\
6710.0690 & 580. \\
6711.2896 & 617. \\
6715.2139 & 632. \\
6719.4971 & 1576. \\
6721.0444 & 2244. \\
6730.3311 & 1243. \\
6738.8252 & 777. \\
6739.0994 & 733. \\
6746.0276 & 611. \\
6747.9980 & 611.
\end{tabular}



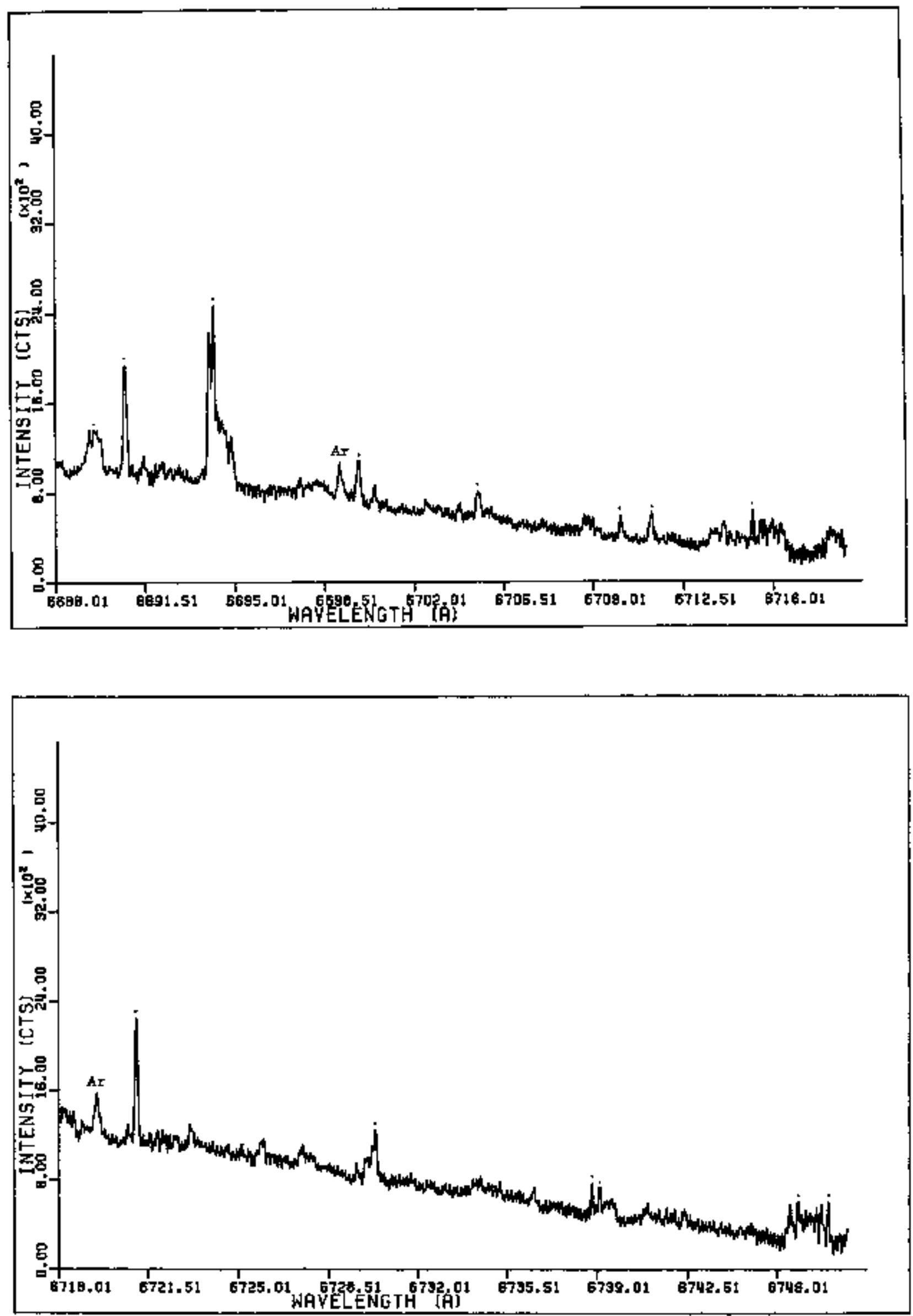


$\begin{array}{cc}\lambda(A) & I \\ 6749+6182 & 1963 . \\ 6752.8345 & 11589 . \\ 6756.2007 & 1781 . \\ 6766.6729 & 1322 . \\ 6782.2227 & 3149 . \\ 6787.0688 & 1937 \\ 6790.5845 & 1414, \\ 6791.8057 & 2440 \\ 6794.8765 & 1075 . \\ 6796.2085 & 1361 . \\ 6797.9102 & 1021 . \\ 6806.1240 & 906\end{array}$



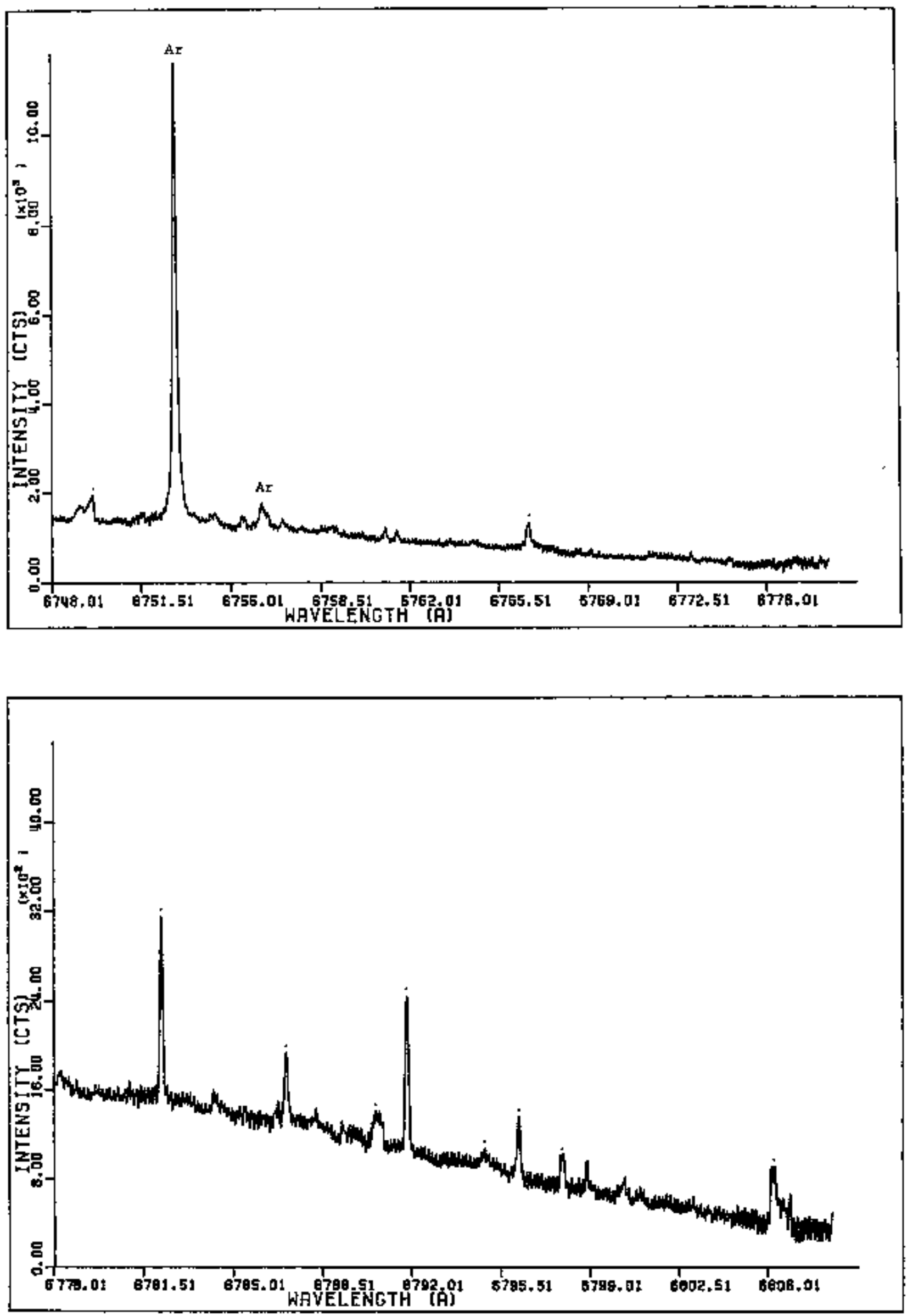


\begin{tabular}{cr}
$\lambda(A)$ & \multicolumn{1}{c}{$I$} \\
6810.3433 & 2050. \\
6817.9561 & 1914. \\
6827.3262 & 1359. \\
6842.9810 & 672. \\
6852.4653 & 1429. \\
6857.9316 & 1352. \\
6864.3760 & 1647. \\
6867.9951 & 12714.
\end{tabular}



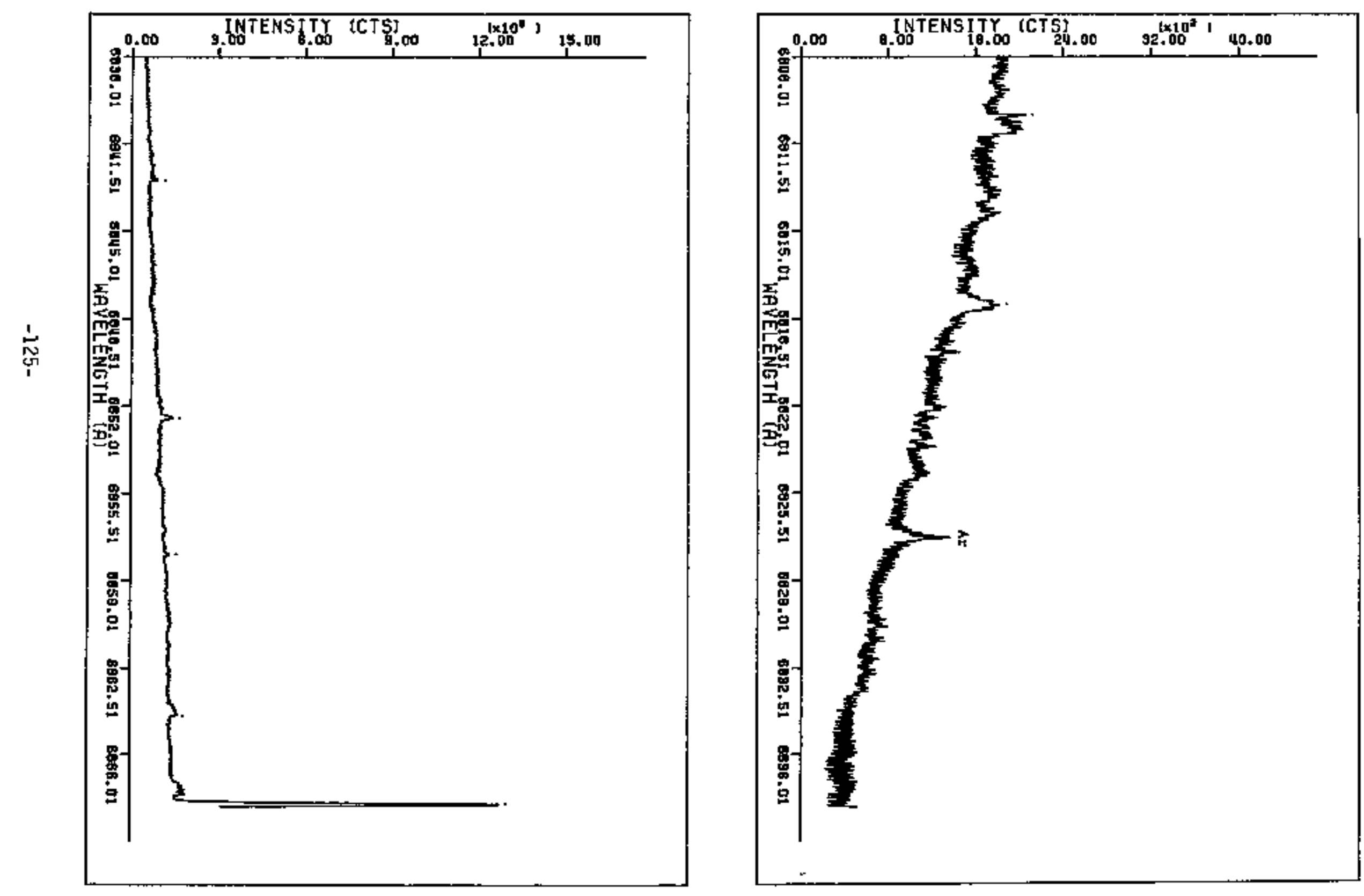


$\begin{array}{cc}\lambda(A) & I \\ 6867.7461 & 9593 . \\ 6868+1035 & 7566 . \\ 6869.3896 & 6052 . \\ 6871.3257 & 7891 . \\ 6979.5952 & 1009 . \\ 6887.1489 & 1118 . \\ 6888.2731 & 1620 . \\ 6895.8481 & 1321 . \\ 6887.1030 & 518 . \\ 6899,1699 & 932 . \\ 6895.7065 & 657 . \\ 6900.6479 & 988 . \\ 6908.3267 & B 43 . \\ 6912,9482 & 986 .\end{array}$



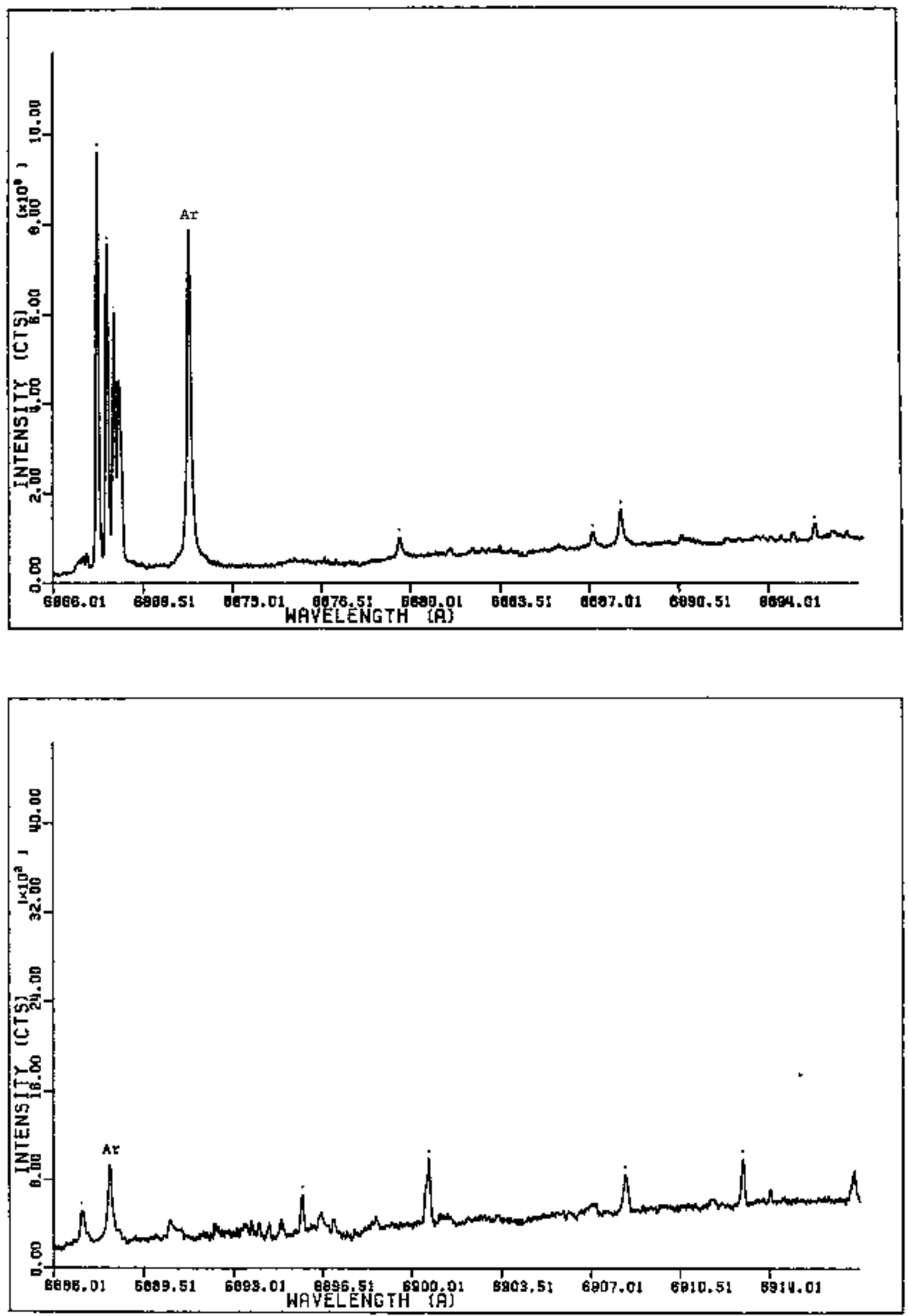


\begin{tabular}{lr}
$\lambda(A)$ & \multicolumn{1}{c}{$I$} \\
6917.2793 & 428. \\
6921.0396 & 590. \\
6922.2002 & 566. \\
6927.5776 & 738. \\
6930.4253 & 710. \\
6932.5342 & 937. \\
6937.6655 & 3526. \\
6941.1460 & 925. \\
6941.6382 & 621. \\
6942.0244 & 724. \\
6944.5557 & 1252. \\
6944.9067 & 1480. \\
6946.9453 & 791. \\
6951.5850 & 771. \\
$6952.902 B$ & 792. \\
6956.0952 & 954. \\
6960.3286 & 817. \\
6965.4644 & 205742. \\
6972.3350 & 1636. \\
6972.5088 & 1842.
\end{tabular}



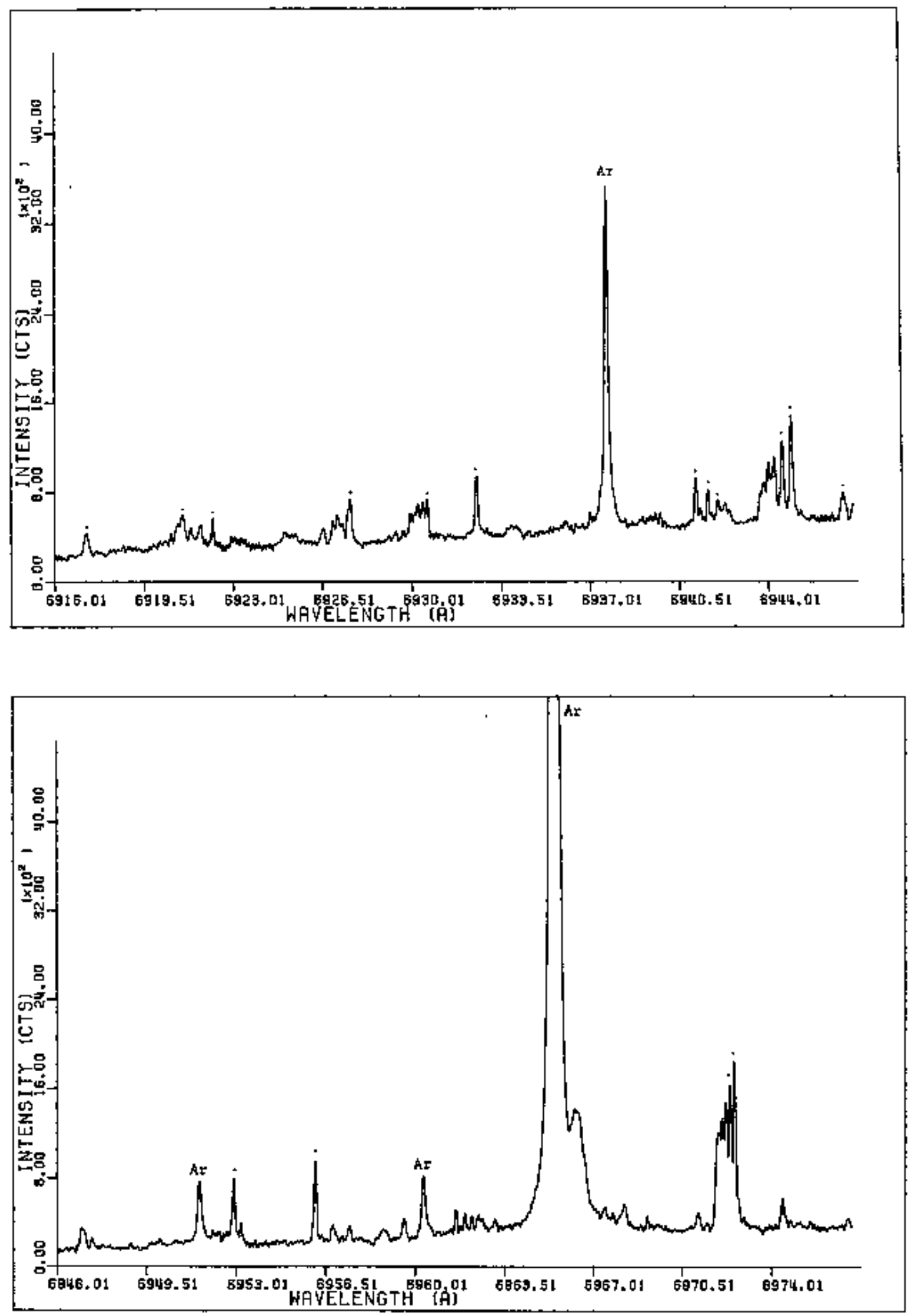


$\begin{array}{ll}\lambda(A) & I \\ 6980.5142 & 305 . \\ 6980.7197 & 370 . \\ 6980.9941 & 439 . \\ 6981.3721 & 494 . \\ 6981.7827 & 655 . \\ 6982.7095 & 313 . \\ 6984.4590 & 303 . \\ 6990+5298 & 490 . \\ 6991.6963 & 329 . \\ 6991.9355 & 366 . \\ 6992.5190 & 450 . \\ 6995,1943 & 667 . \\ 7000.3735 & 336 . \\ 7000.6143 & 302 . \\ 7002.5688 & 747 . \\ 7003.0935 & 331 . \\ 7003.3574 & 351 . \\ 7003.7012 & 361 . \\ 7004.0435 & 344 . \\ 7004.5239 & 344 . \\ 7006.0337 & 307 . \\ 7006.5479 & 447 . \\ 7006.7192 & 564 . \\ 7006.8906 & 765 .\end{array}$




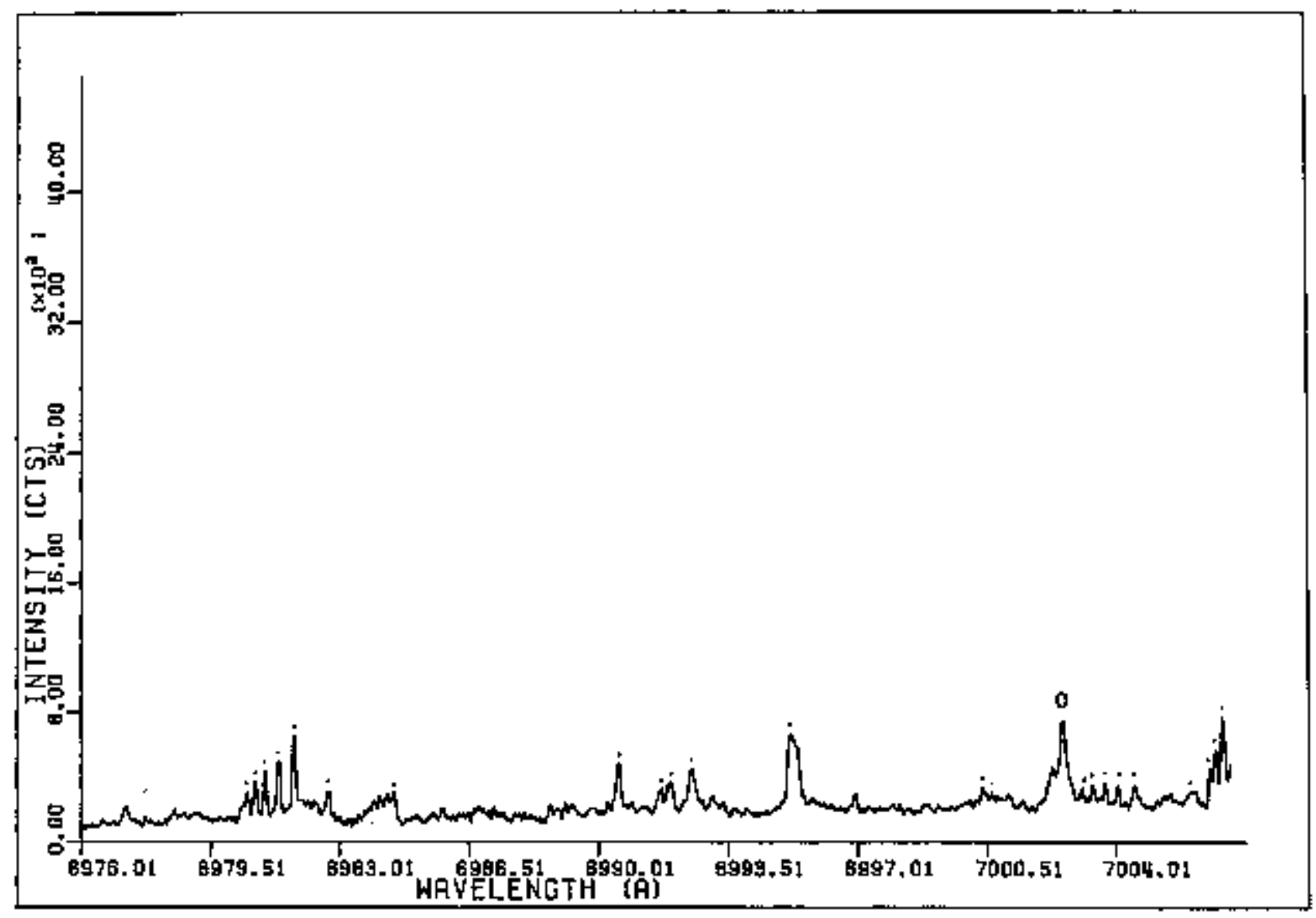


1. Mr. Richard Brown Director

Office of Safeguards and Security, DP-34

U.S. Department of Energy

Washington, D.C. 20545

2. Mr. Glenn Hammono

Director

Division of safeguards, DP-342

office of safeguards and security

U.S. Departient of Energy

Washington, D.C. 20545

3. Mr. William Hagis

Division of Safeguards, DP-342-3

office of safeguards and security

U.S. Department of Energy

Washington, D.C. 20545

4. Mr. Joseph A, Goleb

Division of Safeguards, DP-342-3

office of Safeguards and security

U. S. Department of Energy

Washington, D.C. 20545

5. Mr. Nicholas Marler

Division of Plans and Policy, DP-341-2

office of Safeguards and Security

U. S. Department of Energy

Washington, D.C. 20545

6. Mr. David Camp

Lawtence Livermore Laboratory

P. 0 . Box 808

Livermore, California 94550

7. Dr. C. Bingham

New Brunstick Laboratory

9800 South Casi Averue

Argonne, Illinois 60439

B. Mr. C. Pietri

Physical Science Administrator

U.S. Department of Energy

9800 South Cass Avenue

Argonne, Illinois 60439 
Distribution tist continued

9. Mr, C. Homi

Westinghouse-Hanford Corp.

P. 0 . Box 808

Richland, Washington 99352

10. Mr. W. R. Laing

Oak Ridge National Laboratory

P. 0. Box $x$

Oak Ridge, Tennessee 37830

11. Dr. Martin Edelson

Ames Laboratory

Iowa state University

Ames, Iowa 50011

12. Dr, Velmer Fassel

Ames Laboratory

Iowa state university

Ames , Iowa 50011

13. Mr. David Doda

Westinghouse-Hanford operations

P. 0 . Box 800

Richland, Washington 99352

14. Dr. John Foley

Los Alamos National Laboratory

P. O. Box 1663

Mail stop E540/Group $\Omega-1$

Los Alamos, New Mexico e7545

15. Mr. Lowell Matheson, Head

Safety, Health and Frotection

Ames Laboratory

I owa Sate University

Ames, Iowa 50011

16. Mr. James G. Douglas

Westinghouse-Hanford Operations

P. 0 . Box 800

MS $\mathrm{MO}-037 / 200 \mathrm{~W}$

Richland, Washington 99352

17. Dr. Michael Parsons

Chemistry Division

Los Alamos National Laboratory

Los Alamos, New Mexico 87545

18. Patent office, USDOE

Chicago Operations office

9800 south Cass Avenue

Argonne, Illinois 60439 
Distribution List Continued

19. USDOE-TIC

Oak Ridge Aational Laboratory

P. O. Box 62

Oak Ridge, Tennessee 37930

20. Ws. Beth Weiser

bocument tibrary

Ames Laboratory

Iowa state University

Ames, Iowa 50011

21. Mr. Dave Swindle

Martin Marietta Energy system

P. O. Box P, NS \#273

Oak Ridge, TN 37831

22. Mr. D. D. MeIntyre, Director

Security Division

U. S. Department of Energy

Albuquerque Operations office P. 0. Box 5400

Albuquerque, New Mexico 87115

23. Mr. Don Jewell, Director

Nuclear Safeguards Staff

U.S. Department of Energy

Albuquerque Operations office

P.O. Box 5400

Albuquerque, NM B7115

24. Mr, E. J, McCallum, Director

Safeguards and Security Division

U. S. Department of Energy

Chicago Operations office

9800 South Cass Avenue

Argonne, Illinois 60439

25. Mr. R.\$. Bostian, Director

Safeguards and Security Division

U. S. Department of Energy

Idaho Operations office

550 2nd Street

Idaho Falls, Idaho 83401

26. Mr. E. W. Adams, Director

Safeguards and Security Division

U. S. Department of Energy

Nevada Operations office

P. O. Box 14100

Las Vegas, Nevada 89114 
Distribution list continued

27. Mr. D.J. Cook, Director

Safeguards and Security Division

v. S. Department of Energy

Oak Ridge Operations office

P. O. BOX E

Oak Ridge, Tennessee 37830

28. Mr. C. Hassell, Jr., Director

Nuclear Materials Control Div.

U.S. Department of Energy

Oak Ridge Operations offtce

P.O. BoX E

Oak Ridge, Tennessee 37830

29. Mr. R. H. Jackson, Di rector

Safeguards and security Division

U. S. Departinent of Energy

Richland Operations office

825 Jadwin Avenue

P. O. Box 550

Richiand, Fashington 99352

30. Mr. R.R. Fredlund, Jr., Director

Safeguards and Security Division

u. $s$. Department of Energy

San Francisco Operations office

1333 Broadway

Oakland, California 94612

31. Mr, G, Miserendino, Director

Sofeguards and Security Division

v. S. Department of Energy

Savannah River Operations office P. 0 . Box $\mathrm{A}$

Aiken, South Carolina 29801

32. Mr. Wayne E. Baker, Director

Quality and Materials Division

U.S. Department of Energy

Savannah River operations office P.O. Box A

Aiken, South Carolina 29801

33. Mr, J.A. Bullian, Director

Safeguards and security Division

v. 3 . Department of Energy

Pittsburgh Naval keactors office P. O. Box 109

West Mifflin, Pennsylvania 15122 
Distribution list continued

34. Mr. G. G. Stefani, Jr., Director Safeguards and Security Division u. s. Department of Energy Schenectady Naval Reactors office P. 0. Box 1069

Schenectady, New York 12301

35. Mr. Walter P. Engel

U. 5 . Department of Energy

NE-60

Naval Reactors

Crystal City

washington, D.C. 20585

36. Dr. Ralph Gutmacher

Q-4 Nuclear Safeguards systems Los Alamos National Laboratory P. 0. Box 1663

Los Alamos, New Mexico 87545 